\title{
CSI 2264: Accretion process in classical T Tauri stars in the young cluster NGC 2264^
}

\author{
A. P. Sousa ${ }^{1}$, S. H. P. Alencar ${ }^{1}$, J. Bouvier ${ }^{2,3}$, J. Stauffer ${ }^{4}$, L. Venuti ${ }^{2,3}$, L. Hillenbrand ${ }^{5}$, A. M. Cody ${ }^{6}$, P. S. Teixeira ${ }^{7}$, \\ M. M. Guimarães ${ }^{8}$, P. T. McGinnis ${ }^{1}$, L. Rebull ${ }^{4}$, E. Flaccomio ${ }^{9}$, G. Fürész ${ }^{10}$, G. Micela ${ }^{9}$, and J. F. Gameiro ${ }^{11}$ \\ ${ }^{1}$ Departmento de Física-Icex-UFMG Antônio Carlos, 6627, 31270-90 Belo Horizonte, MG, Brazil \\ e-mail: alana@fisica.ufmg.br \\ 2 Univ. Grenoble Alpes, IPAG, 38000 Grenoble, France \\ 3 CNRS, IPAG, 38000 Grenoble, France \\ ${ }^{4}$ Spitzer Science Center, California Institute of Technology, 1200 East California Boulevard, Pasadena, CA 91125, USA \\ 5 Astronomy Department, California Institute of Technology, Pasadena, CA 91125, USA \\ ${ }^{6}$ NASA Ames Research Center, Kepler Science Office, Mountain View, CA 94035, USA \\ 7 Universität Wien, Institut für Astrophysik, Türkenschanzstrasse 17, 1180 Vienna, Austria \\ 8 Departmento de Física, Universidade Federal de Sergipe, SE 49100-000 Aracaju, Brazil \\ 9 INAF-Osservatorio Astronomico di Palermo, Piazza del Parlamento 1, 90134 Palermo, Italy \\ 10 MIT Kavli Institute for Astrophysics and Space Research, 77 Mass Ave 37-582f, Cambridge, MA 02139, USA \\ 11 Instituto de Astrofísica e Ciências Espaciais and Faculdade de Ciências Universidade do Porto, CAUP, Rua da Estrelas, \\ 4150-762 Porto, Portugal
}

Received 25 May 2015 / Accepted 4 September 2015

\section{ABSTRACT}

\begin{abstract}
Context. NGC 2264 is a young stellar cluster ( 3 Myr) with hundreds of low-mass accreting stars that allow a detailed analysis of the accretion process taking place in the pre-main sequence.

Aims. Our goal is to relate the photometric and spectroscopic variability of classical T Tauri stars to the physical processes acting in the stellar and circumstellar environment, within a few stellar radii from the star.

Methods. NGC 2264 was the target of a multiwavelength observational campaign with CoRoT, MOST, Spitzer, and Chandra satellites and photometric and spectroscopic observations from the ground. We classified the CoRoT light curves of accreting systems according to their morphology and compared our classification to several accretion diagnostics and disk parameters.

Results. The morphology of the CoRoT light curve reflects the evolution of the accretion process and of the inner disk region. Accretion burst stars present high mass-accretion rates and optically thick inner disks. AA Tau-like systems, whose light curves are dominated by circumstellar dust obscuration, show intermediate mass-accretion rates and are located in the transition of thick to anemic disks. Classical T Tauri stars with spot-like light curves correspond mostly to systems with a low mass-accretion rate and low mid-IR excess. About $30 \%$ of the classical T Tauri stars observed in the 2008 and 2011 CoRoT runs changed their light-curve morphology. Transitions from AA Tau-like and spot-like to aperiodic light curves and vice versa were common. The analysis of the $\mathrm{H} \alpha$ emission line variability of 58 accreting stars showed that 8 presented a periodicity that in a few cases was coincident with the photometric period. The blue and red wings of the $\mathrm{H} \alpha$ line profiles often do not correlate with each other, indicating that they are strongly influenced by different physical processes. Classical T Tauri stars have a dynamic stellar and circumstellar environment that can be explained by magnetospheric accretion and outflow models, including variations from stable to unstable accretion regimes on timescales of a few years.
\end{abstract}

Key words. stars: formation - stars: variables: T Tauri, Herbig Ae/Be - open clusters and associations: individual: NGC 2264 accretion, accretion disks

\section{Introduction}

Classical T Tauri stars (CTTSs) are young, low-mass stars $\left(M_{*} \leq\right.$ $\left.2 M_{\odot}\right)$, with spectral types from $\mathrm{F}$ to $\mathrm{M}$. They are surrounded by a circumstellar disk from which they are still accreting material. They present strong and broad emission lines in their spectra and show emission excess with respect to the stellar photosphere that goes from the radio to the ultraviolet (Bouvier et al. 2007b). CTTSs have strong magnetic fields (Johns-Krull et al. 1999; Johnstone et al. 2014) that disrupt the accretion disk at a few stellar radii from the star and channel the accreting material, forming accretion columns. The accreting gas hits the stellar sur-

* Full Tables 2 and 3 are only available at the CDS via anonymous ftp to cdsarc.u-strasbg. fr $(130.79 .128 .5)$ or via

http://cdsarc.u-strasbg.fr/viz-bin/qcat?J/A+A/586/A47 face and creates hot spots. CTTSs also present cold spots at the stellar surface; these are caused by magnetic activity (Bouvier et al. 1995). Part of the gas in the inner disk region is ejected as a wind from the star-disk system along open magnetic field lines that may form collimated jets (e.g., Ferreira et al. 2006). In a few million years, before reaching the main sequence, CTTSs lose their disks and become weak-lined T Tauri stars (WTTSs), which no longer show detectable signs of accretion (e.g., Meyer 2009).

A characteristic of CTTSs is the photometric and spectroscopic variability at various wavelengths. The photometric variability occurs from X-ray to infrared on a timescale from a few minutes to several years and is usually irregular (e.g., Appenzeller \& Mundt 1989). Some stars, however, show periodic behavior, which may be due to the presence of stable 
cold and hot spots on the stellar surface or to circumstellar dust extinction, as observed in the classical T Tauri star AA Tau (Bouvier et al. 2007a; Alencar et al. 2010). The analysis of the photometric variability of CTTSs allows the determination of cold or hot spot characteristics and the typical timescale of the physical processes that cause each type of variability (dynamo, accretion, star-disk interaction). We can also estimate the lineof-sight dust distribution in the inner disk in favorable star-disk inclinations, when the inner disk occults the star as the system rotates. Spectroscopic variations are also present, and emission lines can vary in shape and intensity on a timescale of hours to days (e.g., Johns \& Basri 1995a; Muzerolle et al. 1998; Costigan et al. 2014). The study of the spectroscopic variability of CTTSs can be related to the predictions of magnetospheric accretion models and magnetohydrodynamical simulations, which may include the accretion and the wind components, as well as the stardisk interaction.

CTTS also show infrared excess emission that indicates the presence of a circumstellar disk (e.g., Teixeira et al. 2012). This emission can be used to estimate the amount of dust in the system and relate disk and accretion evolution. The more evolved the star-disk system, the smaller the amount of circumstellar material available, and consequently, the lower the emission excess in the infrared.

Magnetospheric accretion is the standard model to describe the accretion process in CTTS (Shu et al. 1994; Hartmann et al. 1994). Magnetohydrodynamic simulations predict that magnetospheric accretion can occur in stable and unstable regimes (Kurosawa et al. 2008; Kulkarni \& Romanova 2008, 2009; Kurosawa \& Romanova 2013). In the stable regime, accretion occurs through two main accretion funnels (one in each hemisphere), and periodic spectroscopic and photometric variations are expected (Kurosawa \& Romanova 2013), since there is a global organization of the accretion geometry. In the unstable regime, several accretion streams are formed that appear at random locations, creating multiple hot spots on the surface of the star. This accretion regime can be maintained by the Rayleigh-Taylor instability, which acts at the magnetosphere-disk interface (Kurosawa \& Romanova 2013) and causes stochastic photometric and spectroscopic variability. If the Rayleigh-Taylor instability is weak, both the unstable and stable accretion regimes may coexist. Accretion will then occur mainly through two accretion funnels, but random accretion funnels can also appear in the magnetosphere (Kulkarni \& Romanova 2008). These predictions can be checked with photometric and spectroscopic observations of CTTSs that span different timescales, from days to years, as the ones discussed in this work.

We analyze young stars belonging to NGC 2264, a young stellar cluster $(\$ 3 \mathrm{Myr}$ ) located at a distance of $\sim 760 \mathrm{pc}$ from the Sun (Sung et al. 1997). This cluster shows evidence of ongoing star formation, such as the presence of molecular outflows and Herbig-Haro objects. In the pioneering work of Herbig (1954), 84 pre-main sequence stars were found in NGC 2264 with $\mathrm{H} \alpha$ emission and were classified as T Tauri stars. Since then, and because of its proximity and low extinction in our line of sight, NGC 2264 has been the subject of many observational campaigns, from the radio to X-rays (e.g., Rebull et al. 2002; Lamm et al. 2004; Dahm \& Simon 2005; Dahm 2008; Teixeira et al. 2012). About 1000 pre-main sequence stars have already been confirmed as members of NGC 2264 (Sung et al. 2009).

NGC 2264 was observed with the Convection Rotation and planetary Transits (CoRoT) satellite during 23 consecutive days in 2008 (from March 7 to 30), and photometric data with high cadence and high signal-to-noise ratio were obtained for $\sim 300$ known cluster members. The analysis of these data yielded studies in different research areas such as astroseismology (Zwintz et al. 2013), stellar rotation (Affer et al. 2013), binary systems (Gillen et al. 2014), and accretion (Alencar et al. 2010). In the latter, it was shown that the light curve exhibited by the classical T Tauri star AA Tau, which is due to obscuration of the photosphere by circumstellar material in the inner disk region, is common among the cluster members. This opens the possibility of studying the inner disk evolution with photometric variability at different wavelengths. It was also shown that the CoRoT light curve morphology is related to the inner disk evolution and consequently to the accretion process.

A second observational campaign of NGC 2264 was organized in 2011 (Cody et al. 2013), including optical, infrared (IR), and X-ray simultaneous observations of the accreting and non-accreting members of the cluster. We present this in the next section. Using this new data set, we discuss the observed photometric and spectroscopic variability and its relation to magnetospheric accretion model predictions. We classify the CoRoT light curves of accreting systems according to their morphology and compare this classification with accretion diagnostics such as $\mathrm{H} \alpha$ and HeI $6678 \AA$ A emission from FLAMES at the Very Large Telescope (VLT), UV excess from MegaCam at the Canada-France-Hawaii Telescope (CFHT), and disk parameters such as IR excess. A set of non-accreting members of NGC 2264 is used as a control sample to define the role of accretion in the observed correlations. Results from previous observations of NGC 2264 with the CoRoT satellite in 2008 are compared with the 2011 campaign to analyze the dynamical nature of accretion and the inner disk evolution. We analyze the variability in lightcurve morphology between the two CoRoT campaigns and relate it to the proposed stable and unstable accretion scenarios in the literature. Cody et al. (2014) presented a detailed description of the photometric data sets and morphological classification of the various types of CTTS light curves observed with CoRoT and Spitzer in the 2011 campaign.

The paper is organized as follows. In Sect. 2, we present the 2011 observational data of NGC 2264 and the reduction procedures. The CTTS sample selection criteria are presented in Sect. 3. In Sect. 4 we discuss the morphological classification of the CoRoT light curves, following the classification scheme proposed by Alencar et al. (2010). In Sect. 5 we compare the CoRoT light-curve classification to the CFHT photometric analysis undertaken by Venuti et al. (2014). In Sect. 6 we analyze the accretion rate obtained directly from the $\mathrm{H} \alpha$ line flux. In Sect. 7 we morphologically classify the $\mathrm{H} \alpha$ line, as proposed by Reipurth et al. (1996). In Sects. 8 and 9 we obtain the periodicities of the $\mathrm{H} \alpha$ and HeI $6678 \AA$ lines. We discuss the presence of unstable and stable accretion regimes in Sect. 10, and we analyze the correlation between the different $\mathrm{H} \alpha$ line structures (emissions and absorptions) in Sect. 11. In Sect. 12 we discuss some interesting objects to be analyzed individually in a future work. Section 13 presents our conclusions.

\section{Data and reduction}

NGC 2264 was observed with the CoRoT satellite during 40 days, from December 2, 2011 to January 10, 2012, providing exquisite photometry for $\sim 500$ probable cluster members. Simultaneously with the 2011 CoRoT run, the cluster was observed with the Spitzer satellite at 3.6 and $4.5 \mu \mathrm{m}$ for 30 days and for 3.5 days with the Chandra satellite. This campaign 
Table 1. Observations from the Coordinated Synoptic Investigation of NGC 2264.

\begin{tabular}{llll}
\hline \hline Telescope & Instrument & Dates & Bands \\
\hline CoRoT & E2 CCD & 2011-Dec.-1 to 2012-Jan.-3 & $3000-10000 \AA$ \\
VLT & FLAMES/GIRAFFE & 2011-Dec.-4 to 2012-Feb.-29 & $6470-6790 \AA$ \\
CFHT & MegaCam & 2012-Feb.-14 to 2012-Feb.-28 & $u$ and $r$ \\
\hline
\end{tabular}

was called Coordinated Synoptic Investigation of NGC 2264 $\left(\right.$ CSI 2264) ${ }^{1}$, and details about the data acquisition and reduction can be found in Cody et al. (2014). We also obtained $u$ - and $r$-band observations of the cluster with MegaCam/CFHT from February 14 to 28,2012 . They were combined with observations of a first MegaCam campaign in 2010 to yield photometry in the ugri bands for a large sample of stars in the NGC 2264 region. A detailed description of the CFHT observations is given in Venuti et al. (2014).

As a complement to the CSI 2264 campaign, we obtained FLAMES spectra at VLT/ESO of 58 CTTSs and 34 WTTSs. These stars were selected based on the CoRoT light-curve classification of 2008 (Alencar et al. 2010), to include most of the AA Tau and spot-like systems. FLAMES is a high- and medium-resolution multi-object spectrograph, covering a field of view of $25^{\prime}$ in diameter. We obtained 20 to 22 spectra of each target in two different fields, distributed in 20 days from December 2011 to February 2012, part of them simultaneously with the CoRoT observations. We used the HR15N setup for the FLAMES/GIRAFFE spectrograph, centered at $6650 \AA$ and covering the $6470 \AA<\lambda<6790 \AA$ spectral region with a resolution of $R=17000$. This spectral region includes $\mathrm{H} \alpha$, and the HeI $6678 \AA$ spectral lines that are analyzed in detail in the next sections. The observed spectra were reduced using the GASGANO reduction package from ESO. We performed the standard procedure of bias subtraction, flat-field correction, wavelength calibration, and barycentric velocity correction for all spectra and later normalized their continuum. In Table 1, we summarize the observations of the CSI 2264 campaign that are discussed in the following sections.

NGC 2264 is located in front of an emission nebula that is seen spectroscopically as a series of emission lines, such as [NII], [SII], and $\mathrm{H} \alpha$, superposed on the stellar spectra. The $\mathrm{H} \alpha$ nebular contribution is very narrow and in general much smaller than the accretion contribution to the $\mathrm{H} \alpha$ emission of most CTTSs, presenting a mean equivalent width of the $\mathrm{H} \alpha$ nebular contribution of $10.1 \pm 0.5 \AA$, averaged over all the sky fibers. However, in a few spectra, the nebular emission is completely blended with the emission from the star, making it impossible to identify whether a component is of nebular or accretion origin. This sometimes makes removing the nebular emission from the total spectrum challenging. In general, this is a difficult task when using fiber spectrographs, as is the case of FLAMES. The nebular contribution is quite variable across the sky, and the sky fiber closest to the stellar fiber does not always present the same nebular contribution as the stellar fiber. Another difficulty is that some targets are very weak and present a spectrum comparable in intensity to the sky contribution.

To remove the nebular contribution from the stellar spectra, we subtracted the sky spectrum of the nearest sky fiber from the stellar spectrum of each star (see Fig. 1). The subtraction of

\footnotetext{
1 All of the CoRoT and Spitzer light curves can be viewed and downloaded from http://irsa.ipac.caltech.edu/data/ SPITZER/CSI2264/
}
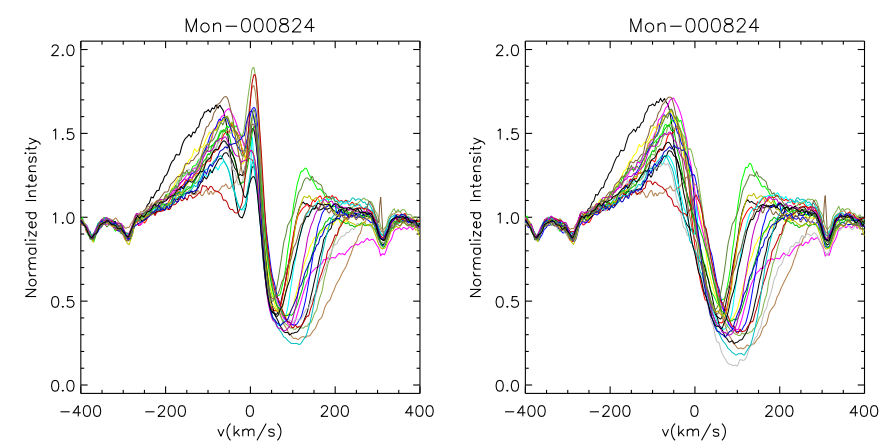

Fig. 1. H $\alpha$ emission lines of a CTTS before (left) and after (right) sky subtraction that show the removal of the nebular emission contribution. The nebular component is the very sharp, narrow emission seen near the line center in the left panel. Different colors correspond to different observing nights.

the nebular emission is sometimes faulty, and a residual nebular line often remains. Even when the sky subtraction leaves residuals in the stellar spectrum, the contamination from the nebula is normally distinguishable from the stellar emission because the sky emission is narrower and weaker than the emission of most CTTSs.

\section{CTTS and WTTS samples}

We selected the CTTSs in NGC 2264 from the known cluster members according to photometric and spectroscopic criteria. We measured the $\mathrm{H} \alpha$ equivalent width $\left(\mathrm{EW}_{\mathrm{H} \alpha}\right)$ and $\mathrm{H} \alpha$ width at $10 \%$ of maximum intensity $\left(\mathrm{W} 10 \%_{\mathrm{H} \alpha}\right)$ in the FLAMES spectra after removing the nebular contribution. We used published values of these parameters for the stars we did not observe spectroscopically, as shown in Table 2. To test the influence of the nebular emission on the measured values of $\mathrm{EW}_{\mathrm{H} \alpha}$, we calculated the $\mathrm{H} \alpha$ equivalent width before and after subtracting the nebular part, from which we obtained a mean difference of about $10 \%$. This difference does not affect our CTTS classification.

According to White \& Basri (2003), CTTS have an $\mathrm{EW}_{\mathrm{H} \alpha}$ higher than a threshold that depends on the stellar spectral type. A young star is considered a CTTS if it presents an $\mathrm{EW}_{\mathrm{H} \alpha}$ higher than $3 \AA$ for K0-K5, $10 \AA$ for K7-M2.5, $20 \AA$ for M3M5.5, and $40 \AA$ for M6-M7.5. Stars that show W10\% ${ }_{\mathrm{H} \alpha}$ higher than $270 \mathrm{~km} \mathrm{~s}^{-1}$ are also considered CTTSs, following White \& Basri (2003). Fang et al. (2009) proposed more stringent limits to separate WTTSs from CTTSs that extend to late-M spectral type. Since we do not have stars in this spectral type region, we decided to use the criteria proposed by White \& Basri (2003) to select CTTS. We selected 220 CTTSs in NGC 2264 that satisfy at least one of the above spectroscopic criteria.

Using UV excess obtained with MegaCam/CFHT, Venuti et al. (2014) found 66 new CTTS candidates in NGC 2264. These stars do not have FLAMES spectra and were not 
Table 2. Parameters of the CTTSs observed by CoRoT and/or FLAMES/VLT.

\begin{tabular}{|c|c|c|c|c|c|c|c|c|c|c|c|c|c|}
\hline Mon ID ${ }^{a}$ & 2 Mass $\mathrm{ID}^{b}$ & $\mathrm{SpT}^{c}$ & $\begin{array}{l}E W_{\mathrm{H} \alpha}{ }^{d} \\
(\AA)\end{array}$ & $\begin{array}{l}\mathrm{Er}_{\mathrm{EW}}{ }^{d} \\
(\AA)\end{array}$ & $\begin{array}{l}\mathrm{W} 10 \%_{\mathrm{H} \alpha}{ }^{d} \\
\left(\mathrm{kms}^{-1}\right)\end{array}$ & $\begin{array}{l}E W_{\mathrm{H} \alpha}{ }^{e} \\
(\AA)\end{array}$ & FUR06 $^{f}$ & $\begin{array}{l}\alpha_{\text {IRAC }}{ }^{g} \\
(2008)\end{array}$ & $\begin{array}{l}\mathrm{LC}^{h} \\
\text { (days) }\end{array}$ & $\begin{array}{l}P^{h} \\
(2011) \\
\end{array}$ & $\begin{array}{l}\mathrm{LC}^{h} \\
\text { (days) }\end{array}$ & $P^{h}$ & $Q^{i}$ \\
\hline CSIMon-000007 & $06415304+0958028$ & K7 & & & & & $\mathrm{c}$ & -1.47 & $3(\mathrm{~A})$ & & $3(\mathrm{~A})$ & & 0.75 \\
\hline CSIMon-000011 & $06411725+0954323$ & K7 & & & & 58.3 & & & $3(\mathrm{~A})$ & & $3(\mathrm{~A})$ & & \\
\hline CSIMon-000017 & $06413199+1000244$ & K5 & & & & 13.1 & & -2.69 & 1 & 4.78 & 1 & 4.73 & -0.03 \\
\hline CSIMon-000021 & $06405944+0959454$ & K5 & & & & 7.4 & $\mathrm{c}$ & & 2 & 3.23 & 2 & 3.16 & 0.15 \\
\hline CSIMon-000056 & $06415315+0950474$ & K5 & & & & 1.8 & w & -1.36 & 2 & 5.71 & 2 & 5.86 & 0.14 \\
\hline CSIMon-000058 & $06420870+0941212$ & $\mathrm{~K} 4.5$ & & & & 94.0 & $\mathrm{c}$ & & - & & 1 & 2.14 & 0.22 \\
\hline CSIMon-000063 & $06411193+0959412$ & M2.5 & & & & 19.4 & & -1.37 & - & & 3 & & \\
\hline CSIMon-000090 & $06410896+0933460$ & M3 & & & & 51.0 & $\mathrm{c}$ & -0.88 & 3 & & 3 & & \\
\hline CSIMon-000103 & $06405954+0935109$ & K6 & 15.6 & 0.9 & 401 & 6.4 & $\mathrm{c}$ & -1.14 & 1 & 1.67 & 1 & 3.35 & 0.21 \\
\hline CSIMon-000117 & $06405413+0948434$ & $\mathrm{M} 2.5$ & & & & 353.0 & & -2.34 & - & & $3(\mathrm{~A})$ & & 0.72 \\
\hline CSIMon-000119 & $06412100+0933361$ & K6 & 10.5 & 0.9 & 466 & 10.6 & $\mathrm{c}$ & -1.44 & 3 & & 3 & & 0.62 \\
\hline
\end{tabular}

Notes. Only a portion of this table is shown here. A full version is available at the CDS. This table is ordered according to the Mon ID. ${ }^{(a)}$ "CSIMon" is an internal identification of the CSI 2264 campaign. Elsewhere in the text "CSI" was omitted for brevity. ${ }^{(b)}$ 2MASS identification. ${ }^{(c)}$ Spectral type obtained by Venuti et al. (2014), Dahm \& Simon (2005), Rebull et al. (2002), Walker (1956). ${ }^{(d)} \mathrm{H} \alpha$ parameters obtained in this work using FLAMES spectra. We used the convention that positive $\mathrm{H} \alpha$ equivalent width indicates $\mathrm{H} \alpha$ in emission, and negative values indicate $\mathrm{H} \alpha$ in absorption. The uncertainties were obtained assuming a Poisson distribution. ${ }^{(e)} \mathrm{H} \alpha$ equivalent width obtained by Dahm \& Simon (2005). ${ }^{(f)}$ Classification as CTTS (c) and WTTS (w) by Fúrész et al. (2006). ${ }^{(g)} \alpha_{\text {IRAC }}$ is the slope of the spectral energy distribution between $3.6 \mu$ m and $8 \mu \mathrm{m}$ obtained by Teixeira et al. (2012). ${ }^{(h)}$ CoRoT light curve morphology and photometric period obtained in this work: " 1 " = spot-like, " 2 " = AA Tau-like, "3" = non-periodic light curves. Accretion bursts and aperiodic extinction light curves are identified by (A) and (E), respectively. "UNC" = unclassified light curve and "B" = Binary. ${ }^{(i)}$ Parameter defined by Cody et al. (2014) to distinguish periodic from aperiodic light curves. According to Cody et al. (2014), when $Q>0.6$ the star is not periodic photometrically.

Table 3. Parameters of the WTTSs observed by CoRoT and/or FLAMES/VLT.

\begin{tabular}{|c|c|c|c|c|c|c|c|c|}
\hline Mon $\mathrm{ID}^{a}$ & 2 Mass ID $^{b}$ & $\mathrm{SpT}^{c}$ & $\begin{array}{l}E W_{\mathrm{H} \alpha}{ }^{d} \\
(\AA)\end{array}$ & $\begin{array}{l}\operatorname{Er}_{\mathrm{EW}}{ }^{d} \\
(\AA)\end{array}$ & $\begin{array}{l}\mathrm{W} 10 \%_{\mathrm{H} \alpha}{ }^{d} \\
\left(\mathrm{kms}^{-1}\right)\end{array}$ & $\begin{array}{l}E W_{\mathrm{H} \alpha}{ }^{e} \\
(\AA)\end{array}$ & FUR06 ${ }^{f}$ & $\alpha_{\text {IRAC }}^{g}$ \\
\hline CSIMon-000008 & $06414859+0954115$ & K5 & & & & & & \\
\hline CSIMon-000009 & $06420914+0948048$ & F5 & & & & & & \\
\hline CSIMon-000014 & $06420664+0941317$ & K7:M0 & & & & & & \\
\hline CSIMon-000015 & $06420911+0959027$ & K7:M0 & & & & & & \\
\hline CSIMon-000018 & $06411322+0955086$ & $\mathrm{~K} 3: \mathrm{K} 4$ & & & & 3.0 & & -2.73 \\
\hline CSIMon-000020 & $06420924+0944034$ & K7 & & & & & $\mathrm{w}$ & \\
\hline CSIMon-000022 & $06410457+1000426$ & M4 & & & & & & \\
\hline CSIMon-000024 & $06415684+0947451$ & M1 & & & & & & \\
\hline CSIMon-000026 & $06410518+1000189$ & M4 & & & & 6.5 & & \\
\hline CSIMon-000029 & $06410328+0957549$ & K7 & & & & 1.5 & & -2.85 \\
\hline CSIMon-000033 & $06410726+0958311$ & K5 & & & & 1.4 & $\mathrm{w}$ & -2.78 \\
\hline
\end{tabular}

Notes. Only a portion of this table is shown here. A full version is available at the CDS. This table is ordered according to the Mon ID. (a) "CSIMon" is an internal identification of the CSI 2264 campaign. ${ }^{(b)}$ 2MASS identification. ${ }^{(c)}$ Spectral type obtained by Venuti et al. (2014), Dahm \& Simon (2005), Rebull et al. (2002), Walker (1956). ${ }^{(d)} \mathrm{H} \alpha$ parameters obtained in this work using FLAMES spectra. We used the convention that positive $\mathrm{H} \alpha$ equivalent width indicates $\mathrm{H} \alpha$ in emission, and negative values indicate $\mathrm{H} \alpha$ in absorption. The uncertainties were calculated assuming a Poisson distribution. ${ }^{(e)} \mathrm{H} \alpha$ equivalent width obtained by Dahm \& Simon (2005). ${ }^{(f)}$ Classification as CTTS (c) and WTTS (w) by Fúrész et al. (2006). ${ }^{(g)} \alpha_{\text {IRAC }}$ is the slope of the spectral energy distribution between $3.6 \mu \mathrm{m}$ and $8 \mu \mathrm{m}$ obtained by Teixeira et al. (2012).

previously classified as CTTS in the literature or are unknown NGC 2264 members, but since they present considerable UV excess with respect to the WTTS locus, they were added to our CTTS list. This means that based on photometric and spectroscopic accretion criteria, 286 stars are thought to be CTTSs in NGC 2264.

Mon-000056 and Mon-000358 were initially not included in our CTTS list, but they show CoRoT light curves in 2008 and/or 2011 that resemble the AA Tau light curve (see Sect. 4). We would therefore expect them to be CTTSs, but they were not in the fields of our FLAMES observations and show little UV excess. These stars were nevertheless observed with FLAMES/GIRAFFE as part of the Gaia/ESO public spectroscopic survey (Randich et al. 2013; Gilmore et al. 2012). We downloaded the spectra from the ESO science archive and reduced them following the same steps as listed in Sect. 2. The
Gaia/ESO spectra show that Mon-000056 presents $E W_{\mathrm{H} \alpha}=$ $2.9 \AA$ and $\mathrm{W} 10 \%_{\mathrm{H} \alpha}=276 \mathrm{~km} \mathrm{~s}^{-1}$, while Mon-000358 shows $E W_{\mathrm{H} \alpha}=32.8 \AA$ and $\mathrm{W} 10 \%_{\mathrm{H} \alpha}=395 \mathrm{~km} \mathrm{~s}^{-1}$, which allows us to classify them as CTTSs. Our final CTTS list therefore contains 288 stars; it is shown in Table 2. Of these, 58 stars were observed by FLAMES and 157 were observed by CoRoT in the 2008 and/or 2011 campaign, including 12 CTTSs observed by CoRoT only in 2008, 63 only in 2011, and 84 CTTSs that were observed in both campaigns.

We also selected 431 WTTSs among the confirmed members of NGC 2264 that we used as a comparison sample to the accreting CTTS. We added 257 WTTSs to the list based on the $\mathrm{H} \alpha$ equivalent width or $\mathrm{W} 10 \%_{\mathrm{H} \alpha}$ criteria of White \& Basri (2003), previously discussed, and 174 WTTSs were selected based on the criteria discussed in Venuti et al. (2014). Information about the WTTS sample can be found in Table 3. Of 

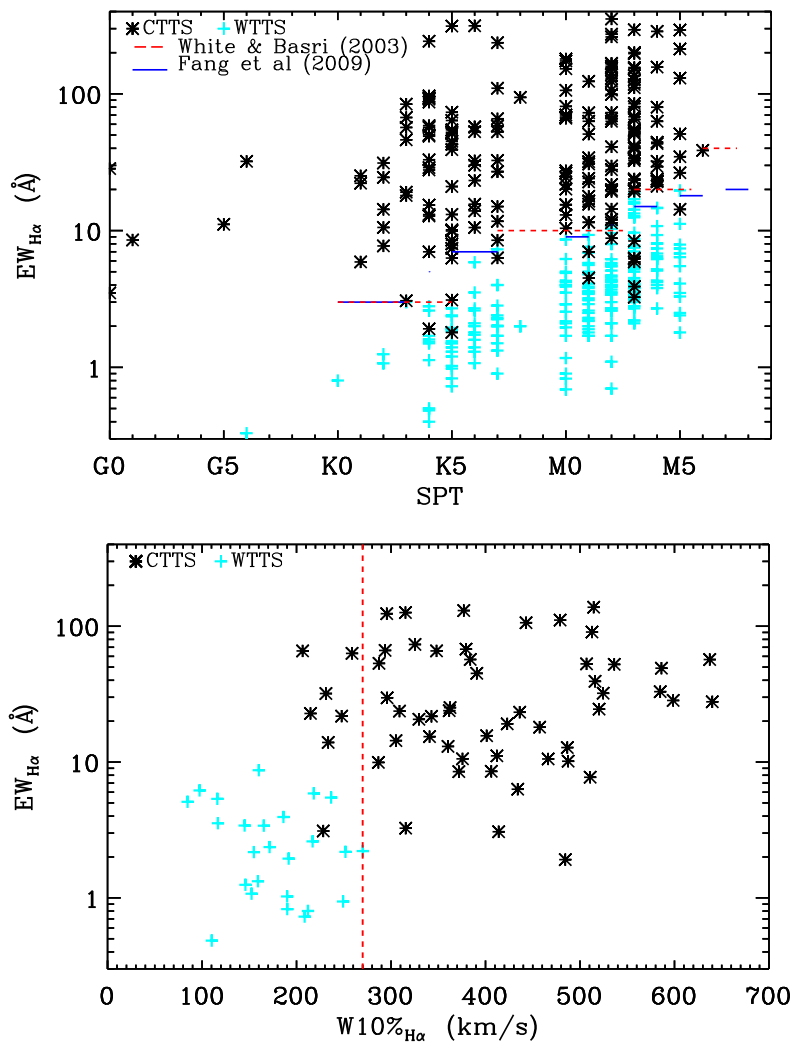

Fig. 2. Spectroscopic criteria used to select CTTSs and WTTSs in NGC 2264. CTTSs are shown as asterisks and WTTSs as plus signs. Top: $\mathrm{H} \alpha$ equivalent width vs. spectral type for our sample of stars. Dashed horizontal lines represent the White \& Basri (2003) classification threshold and solid horizontal lines the Fang et al. (2009) proposed criteria. Bottom: $\mathrm{H} \alpha$ equivalent width vs. $\mathrm{H} \alpha$ width at $10 \%$ of maximum intensity. The dashed vertical line is drawn at $270 \mathrm{~km} \mathrm{~s}^{-1}$, the separation between CTTSs and WTTSs, according to White \& Basri (2003). A few stars do not satisfy one spectroscopic CTTS criterion, but they were classified as CTTSs either based on the other spectroscopic criterion or on the UV excess.

the 431 WTTSs, 34 were observed by FLAMES and 308 stars were observed by CoRoT in the 2008 and/or 2011 campaign. We did not analyze the CoRoT data for WTTSs; that is beyond the scope of this paper.

In Fig. 2, we plot the spectroscopic criteria used to distinguish CTTSs from WTTSs, as proposed by White \& Basri (2003) and Fang et al. (2009), together with the $\mathrm{H} \alpha$ data for the 58 CTTS and 34 WTTS observed with FLAMES. The spectroscopic data of the stars that were not observed with FLAMES come from Dahm \& Simon (2005). A few stars do not satisfy the $\mathrm{EW}_{\mathrm{H} \alpha}$ CTTS criteria, but they were classified as CTTSs based on $\mathrm{W} 10 \%_{\mathrm{H} \alpha}$ (Fig. 2, bottom panel) and/or UV excess.

\section{Morphology of the CoRoT light curves}

The 2008 CTTS light curves were previously classified morphologically as spot-like, AA Tau-like, or non-periodic by Alencar et al. (2010). The spot-like light curves display sinusoidal variations that are due to large, cold spots at the stellar surface, which are very stable on the timescale of the observations (about three weeks). The AA Tau-like light curves show a well -defined maximum interrupted by periodic minima that vary in width and depth from cycle to cycle, like AA Tau itself. They correspond to systems whose light-curve variability is caused by a periodic

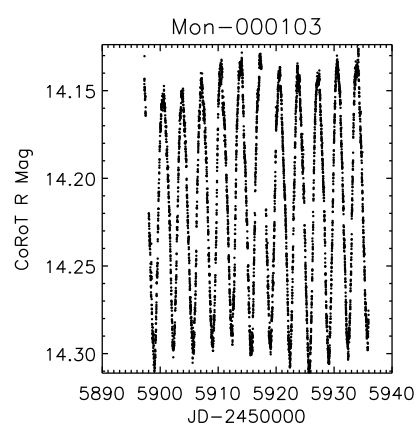

(a)

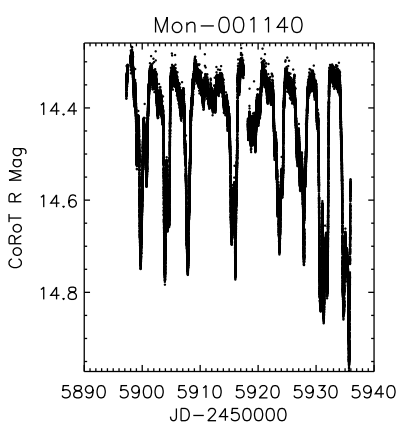

(c)

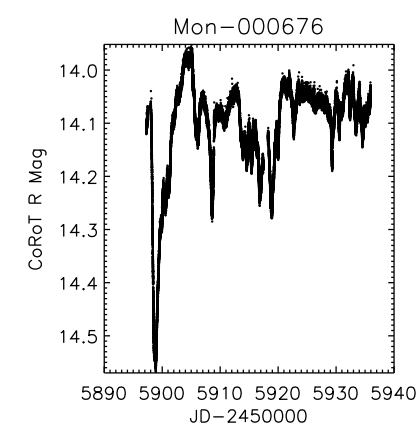

(e)

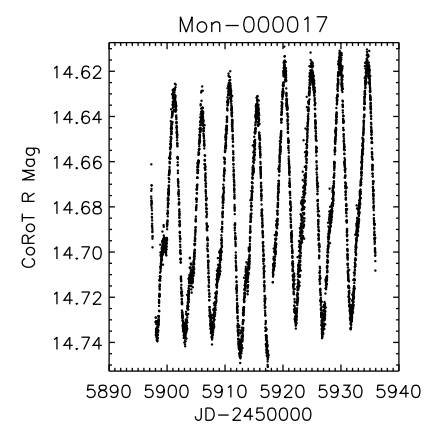

(b)

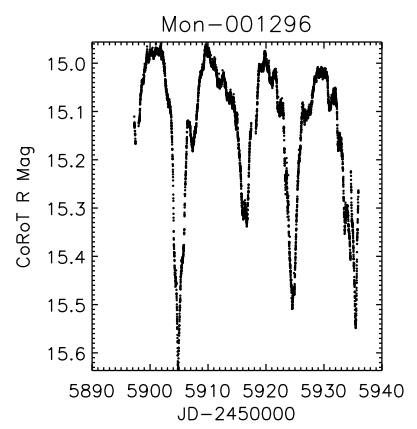

(d)

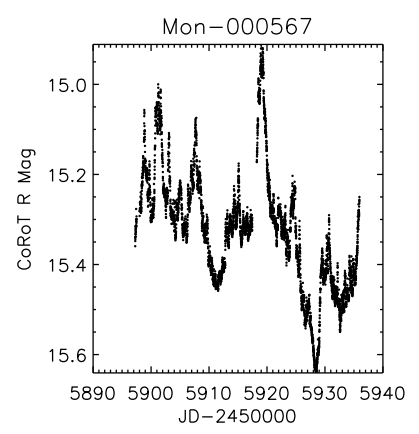

(f)
Fig. 3. CoRoT light curves of CTTSs from 2011 showing different photometric variabilities. Panels a) and b) correspond to spot-like light curves, c) and d) to AA Tau-like light curves, e) is a non-periodic light curve caused by obscuration by circumstellar material, and f) is a non-periodic light curve dominated by accretion bursts. The lightcurve magnitude was calibrated using the $R$ filter with a zero point of $26.74 \mathrm{mag}$, as described in Cody et al. (2014).

occultation of the stellar photosphere by circumstellar dust (see McGinnis et al. 2015; Alencar et al. 2010, for more information about AA Tau-like stars in NGC 2264 and Fonseca et al., in prep. for a specific AA Tau-like system, V354 Mon - Mon-000660). Non-periodic light curves may be due, for example, to accretion bursts (Stauffer et al. 2014) or random circumstellar dust obscuration. Some non-periodic light curves are difficult to assign to a major physical phenomenon, however, and are probably the result of different superposed variability processes, such as variable accretion, dust obscuration, and spot variations. We show below by comparing our light-curve classification with accretion diagnostics and inner disk parameters that the morphological classification of the CoRoT light curves is related to the evolution of the accretion process and of the inner disk region.

We morphologically classified the 145 CTTSs light curves observed in 2011 by CoRoT, and in Fig. 3 we show examples 


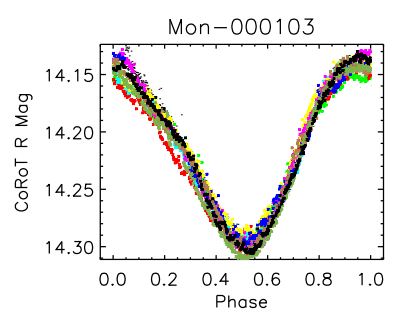

(a)

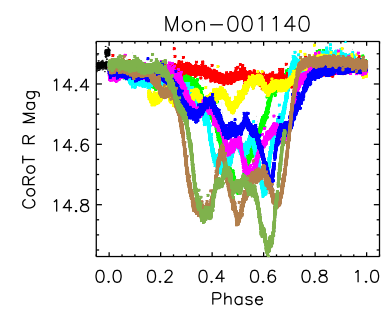

(c)

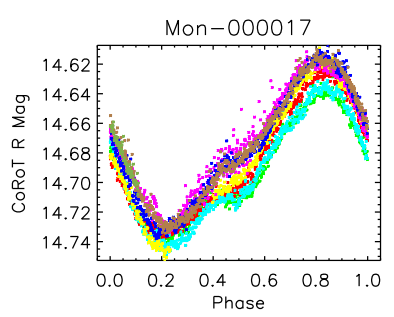

(b)

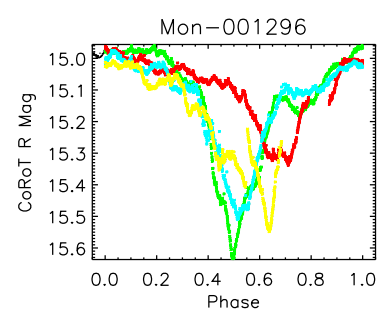

(d)
Fig. 4. Periodic CoRoT light curves from Fig. 3 folded in phase. The spot-like light curves present stable variations (top) that contrast with the high variability of the AA Tau-like light curves (bottom). Different colors represent different rotational cycles.

Table 4. Morphological classification of CTTS light curves observed with CoRoT in 2008 and 2011.

\begin{tabular}{lcc}
\hline \hline Light curve type & No. of stars-2008 & No. of stars-2011 \\
\hline \multicolumn{3}{c}{ Periodic or quasi-periodic } \\
\hline Spot & 24 & 19 \\
AA Tau & 15 & 18 \\
Eclipsing binary & 2 & 2 \\
Unclassified & 0 & 4 \\
\hline \multicolumn{3}{c}{ Non-periodic } \\
\hline Random extinction & 13 & 13 \\
Accretion bursts & 12 & 20 \\
Unclassified & 30 & 69 \\
\hline Total & 96 & 145 \\
\hline
\end{tabular}

of these light curves. In Fig. 4, we present the periodic light curves of Fig. 3 folded in phase with the period obtained with the Scargle periodogram, as modified by Horne \& Baliunas (1986). The stability of the spot-like light curve modulation is evident, which contrasts with the high variability of the typical AA Tau-like light curves. A detailed statistical and morphological analysis of the light curves of stars that present infrared excess observed in our campaign with CoRoT and Spitzer are discussed in Cody et al. (2014), where the light curves are separated into twelve different classes that represent diverse physical processes and geometric effects. We list in the last column of Table 2 the $Q$ parameter defined by Cody et al. (2014) that can be used as a metric to distinguish periodic and quasi-periodic $(Q<0.6)$ from aperiodic $(Q>0.6)$ light curves.

Table 4 shows some morphological statistics of the CTTS light curves observed with CoRoT in 2008 and 2011. The number of periodic CTTSs did not vary considerably from one run to the other. However, the number of CTTSs observed with CoRoT increased in 2011, and more non-periodic stars were observed in 2011 than in 2008. This was expected, since most of the known periodic variable systems were already included in the 2008 run. The number of AA Tau-like systems identified in 2008
Table 5. Variations of the morphological classification of the 84 CTTS light curves observed with CoRoT in 2008 and 2011.

\begin{tabular}{lc}
\hline \hline Light curve $2008 \rightarrow$ Light curve 2011 & No. of stars \\
\hline Spot $\rightarrow$ AA Tau & 0 \\
Spot $\rightarrow$ non-periodic & 9 \\
AA Tau $\rightarrow$ spot & 0 \\
AA Tau $\rightarrow$ non-periodic & 5 \\
Non-periodic $\rightarrow$ AA Tau & 8 \\
Non-periodic $\rightarrow$ spot & 2 \\
Non-periodic $\rightarrow$ periodic unclassified & 1 \\
AA Tau $\rightarrow$ AA Tau & 8 \\
Spot $\rightarrow$ spot & 12 \\
Non-periodic $\rightarrow$ non-periodic & 37 \\
Binary $\rightarrow$ binary & 2 \\
\hline
\end{tabular}

differs from that of Alencar et al. (2010), since we were able here to use the simultaneous FLAMES spectroscopy to refine our classification. In addition, Stauffer et al. (2015) identified a new subclass of stars with variable extinction light curves that show periodic, shallow, and short-duration flux dips that are approximately Gaussian in shape. There is some overlap between this new class and the classical AA Tau systems, since a few AA Tau-like stars also present narrow dips superposed on the broad deep minima, as explained in their paper. Light curves labeled "unclassified" in Table 4 correspond to the light curves that we were unable to clearly associate with one major physical phenomenon.

\subsection{Morphological changes from 2008 to 2011}

Several stars exhibited the same light-curve morphology in 2008 and 2011 , but $\sim 30 \%$ of the stars presented variations in the morphology of their light curves. We show in Table 5 the variation of morphological classification of the 84 CTTS light curves that were observed in both campaigns. The transition from AA Tau to non-periodic classification and vice versa is common, but we did not observe a transition from AA Tau to spot-like or from spot-like to AA Tau. A few spot-like systems became irregular, and the opposite also occurred. In Figs. 5a and b, we show the 2008 and 2011 light curves of the CTTS Mon-000928, whose light-curve classification changed between the two runs. In 2008, Mon-000928 was classified as an AA Tau-like system with a maximum brightness periodically interrupted by minima, while in 2011 it was clearly irregular with no detected periodicity. These variations show that the inner disk structure that is responsible for the stellar occultation can move from a wellorganized geometry with a stable inner disk warp to a random dust distribution in just a few years. These results may also hint at the typical timescale of stability of the accretion regime, where the accretion geometry evolves from a main accretion funnel in each hemisphere, the base of which may correspond to the stable inner disk warp to an unstable accretion scenario, where random accretion funnels are formed (Kurosawa \& Romanova 2013; McGinnis et al. 2015).

In young low-mass stars, cold spots often occupy a substantial fraction of the stellar surface and may last for months to years (e.g., Herbst 1989; Vogt et al. 1999). If the spots indeed did not evolve significantly over a period of few years, then the light curves would also be substantially the same in our two epochs. However, in our sample, 9 of 21 spot-like light curves, observed in both epochs, changed their morphology from 2008 to 2011 and became aperiodic, as seen in Table 5. At the same 


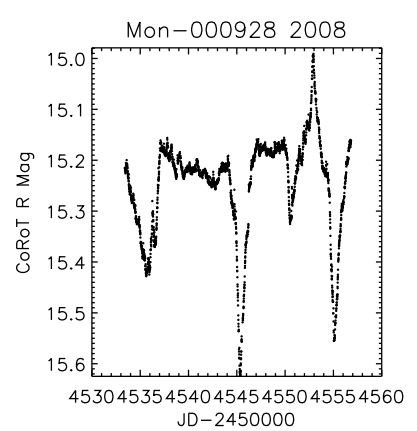

(a)

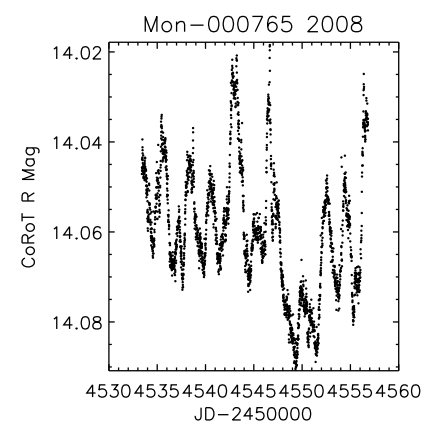

(c)

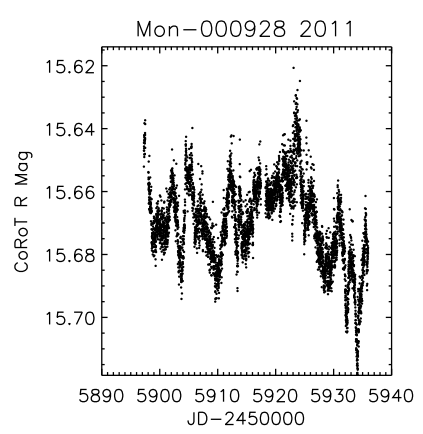

(b)

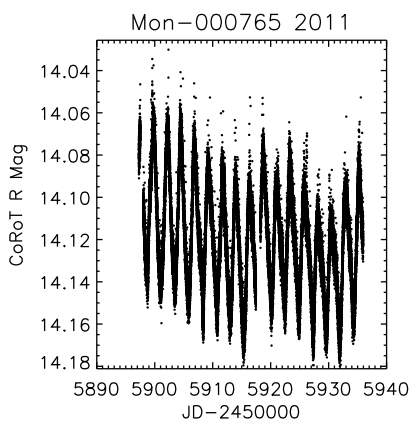

(d)
Fig. 5. Examples of CTTSs whose morphological classification of the light curve varied from 2008 to 2011. Mon-000928 displays an AATau-like light curve in 2008 a) with a period of $P=4.96$ days, while in 2011 the light curve was aperiodic b). Mon-000765 was aperiodic in 2008 c), while in 2011 the light curve was spot-like with a period of $P=2.41$ days $\mathbf{~ d}$ ).

time, 2 aperiodic light curves in 2008 became spot-like in 2011. In a study of the Orion nebula cluster (ONC), Rebull (2001) analyzed Cousins $I_{\mathrm{C}}$ light curves from young stars and showed that in a one-year interval, about half of the observed periodic spotlike light curves became aperiodic. They also compared their measured periods with the results of similar analyses of the ONC by other authors (Stassun et al. 1999; Herbst et al. 2000) and showed that only about $50 \%$ of the periodic systems are typically recovered between the different works. The results above show that cold spots in young stars can evolve substantially and that the observable characteristic features, such as light-curve periodicity, can disappear on a timescale of a few years. Cool spots are expected to be present in all young low-mass stars, but only some of the CTTSs present periodic spot-like light curves, since overlying accretion hot spots and circumstellar dust obscuration can effectively mask cool spot signatures. When CTTSs are observed to switch between variability categories, it is generally attributable to changes in accretion behavior on timescales of a few years or less. In Figs. 5c and d, we present the 2008 and 2011 light curves of the CTTS Mon-000765, whose light-curve classification changed from aperiodic to spot-like.

Figure 5 shows that flux variations can also be observed from 2008 to 2011 in the spot-like stars that presented a variable light-curve periodicity. During the periodic epoch, most spotlike systems were fainter than in the aperiodic cycles, as seen in Mon-000765 (Fig. 5, lower panels). The brighter phase during the irregular cycles could be due to accretion bursts, leading to the appearance of randomly distributed bright spots at the stellar surface, which would at the same time explain the irregular photometric variability. The AA Tau-like stars also show flux variability between the periodic and aperiodic phases, but in the opposite sense as compared to the spot-like systems: they are generally brighter when periodic, as seen in Mon-000928 (Fig. 5, top panels). These systems are observed at high inclinations with respect to our line of sight $\left(i>60^{\circ}\right.$, see McGinnis et al. 2015), and the aperiodic cycles may correspond to the unstable accretion regime described by Kurosawa et al. (2008) and Kurosawa \& Romanova (2013), where multiple accretion streams are randomly formed and occult the star as the system rotates. In this scenario, the star can be partially occulted at all rotational phases, never exhibiting its maximum flux to the observer, and being therefore fainter than in the stable and ordered AA Tau phase. This is consistent with the fact that, in the aperiodic cycles, we generally do not observe a well-defined maximum in the light curves, while in the periodic AA Tau phase a clear maximum is seen, which should correspond to the unocculted photospheric brightness.

\subsection{Period distribution}

Table 4 shows that $\sim 43 \%$ of the 96 CTTSs observed in 2008 and $\sim 30 \%$ of the 145 CTTSs observed in 2011 were classified as periodic. We only classified as periodic the light curves that presented a high-power, detached, and dominant peak in the periodogram analysis. We established a minimum power level of the periodogram for a reliable detection based on the periodogram analysis of the sinusoidal spot-like light curves, which are clearly periodic with a well-determined period within our dataset. We also inspected by eye each of the periodic light curves folded in phase at the measured period and verified that they presented phase coherence.

Many light curves that show some periodic signal, but with low power in the periodogram, were conservatively classified as non-periodic in the present work. Venuti et al. (in prep.) discuss the peridicity of all the stars observed by CoRoT in more detail and the number of periodic stars may be somewhat different from ours. We present in Fig. 6 the period distribution of AA Tau-like and spot-like systems observed in 2008 and 2011. Despite the observed morphological variations in the light curves from 2008 to 2011 (Table 5), the overall period distribution did not vary significantly from 2008 to 2011 . As seen in Fig. 6, there are more AA Tau-like stars with long photometric periods in the new campaign. This is probably due to the the difference in datasets between the two campaigns and to the longer observation time span in 2011 (40 days) than in 2008 ( 23 days), which makes it easier to confidently determine longer periods in the irregular, though periodic, AA Tau-like light curves. Despite the longer observational time span, we did not detect longer spotlike periods in 2011. In 2008 and 2011 the spot-like light curves were very regular and their periods were easily measured with our data. Therefore, the lack of periods longer than 14 days in 2008 and 10 days in 2011 is probably real among the observed CTTSs. The longer periods measured were different in both campaigns, and this is due to different samples, since the stars observed in each run were not exactly the same, and it is due to accretion variability, since some spot-like systems became aperiodic and the opposite also occurred between the two observational runs.

The period of a spot-like light curve is the rotational period of the star, since spots are located on the stellar surface. In both epochs, the AA Tau-like stars presented periods within the range of periods measured in the spot-like light curves. Therefore, the material that obscures the AA Tau-like stars is probably not located very far from the corotation radius of the star-disk system, 

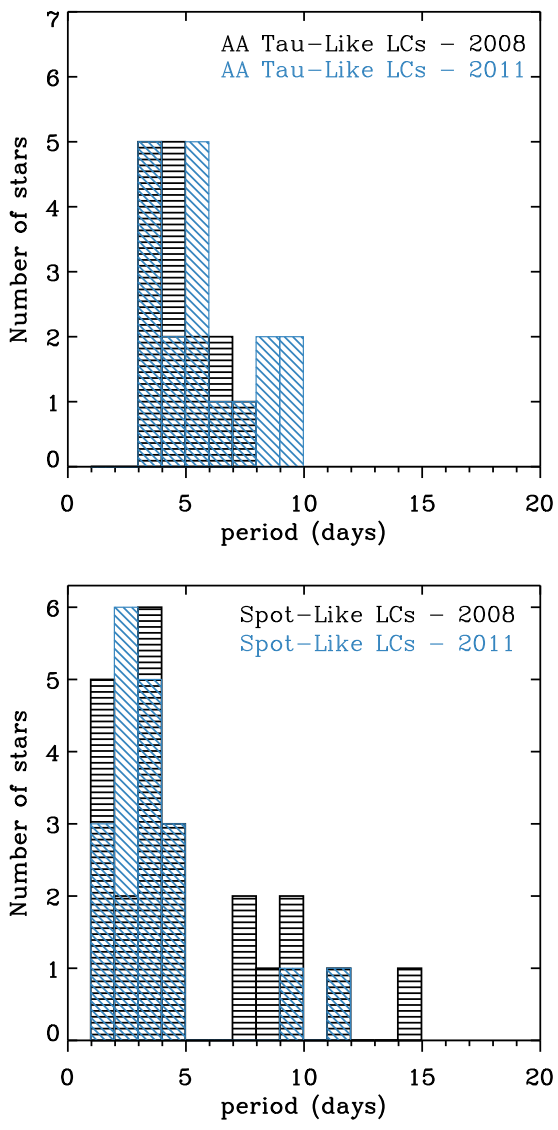

Fig. 6. Period distribution of the CTTSs observed in 2008 (black) and 2011 (blue). Top: AA Tau-like light curves. Bottom: spot-like light curves. AA Tau-like stars clearly present periods within the range of spot-like systems.

as pointed out by Alencar et al. (2010). A more detailed analysis of the period distribution of CTTSs and WTTSs in NGC 2264 and the implication for angular momentum evolution will be presented in another paper (Venuti et al., in prep.).

\section{UV excess and photometric mass-accretion rates}

In this section, we compare the CoRoT light-curve morphology with accretion diagnostics and disk indicators to determine whether the optical light-curve morphology and accretion and inner dusty disk evolution are related.

Venuti et al. (2014) obtained the UV excess emission of CTTSs and WTTSs in NGC 2264 using $u$ - and $r$-band measurements from CFHT data. They determined the locus of WTTSs in the $r$ vs. $u-r$ color-magnitude diagram that was fitted with a polynomial function, which was used as a reference value to non-accreting systems. The UV excess of a CTTS was defined as $E(u)=(u-r)_{\mathrm{obs}}-(u-r)_{\mathrm{ref}}$, where $(u-r)_{\mathrm{obs}}$ is the observed CTTS color, and $(u-r)_{\text {ref }}$ is the reference color at magnitude $r_{\text {obs }}$. The UV excess measurements have an average rms error of 0.16 mag to account for the distribution of WTTS around the reference sequence (Venuti et al. 2014). With the UV excess, Venuti et al. (2014) calculated mass-accretion rates $\left(\dot{M}_{\mathrm{UV}}\right)$ for the CTTSs in NGC 2264. We show in Fig. 7 the UV excess (top) and mass-accretion rate (bottom) vs. $\mathrm{H} \alpha$ equivalent width for the stars in our sample. Stars presenting accretion burst light curves show some of the highest UV excesses (more negative on the scale of the plot) and consequently the highest mass-accretion
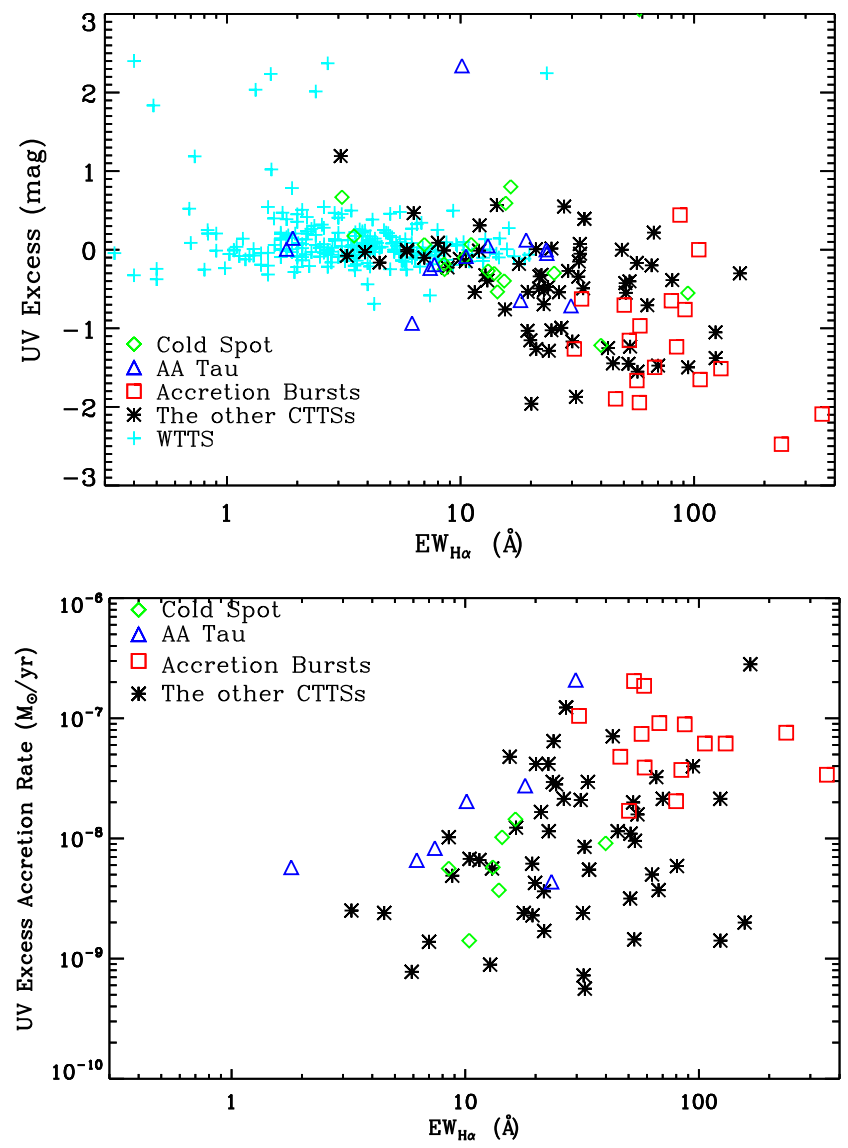

Fig. 7. Comparison between the CoRoT light-curve classification and UV excess (top) or mass-accretion rates (bottom) calculated by Venuti et al. (2014). In the top plot, more negative values indicate higher UV excess.

rates among the observed systems, as noted by Stauffer et al. (2014). This is supported by a two-sided Kolmogorov-Smirnov test that shows that the mass-accretion rate distribution of accretion burst stars, when compared to spot-like and AA Tau-like systems, has only a $5.8 \times 10^{-6}$ probability to be equivalent. AA Tau-like and spot-like stars are an intermediate population between the accretion bursts systems and WTTSs, which present low UV excess. The mean UV excess values of each light curve group corroborate with this analysis: -1.15 for the accretion bursts, -0.04 for the AA Tau systems, 0.05 for the spot-like systems, and 0.14 for the WTTSs. These results suggest that the CoRoT light curve morphology is related to the evolution of the accretion process.

Many of the stars observed by CoRoT have Spitzer observations from the literature (Teixeira et al. 2012), which allowed us to compute the $\alpha_{\text {IRAC }}$ index, as proposed by Lada et al. (2006). This index is the slope of the spectral energy distribution between $3.6 \mu \mathrm{m}$ and $8 \mu \mathrm{m}$, and it allows a classification of inner disk evolution. If $\alpha_{\text {IRAC }}<-2.56$, the disk is classified as a naked photosphere (no dust in the inner disk). For systems with $-2.56<\alpha_{\text {IRAC }}<-1.80$, the inner disk is optically thin and called an anemic disk. For stars with $-1.80<\alpha_{\text {IRAC }}<$ -0.5 , the inner disk is classified as optically thick. Stars with $-0.5<\alpha_{\text {IRAC }}<0.5$ are classified as flat spectrum sources, and $\alpha_{\text {IRAC }}>0.5$ characterizes class I sources.

In Fig. 8, we show the $\alpha_{\text {IRAC }}$ index vs. the UV excess calculated by Venuti et al. (2014). Most of the CTTSs present dust in the inner disk. Spot-like systems are present in all the inner disk 
A. P. Sousa et al.: CSI 2264: Accretion process in classical T Tauri stars in the young cluster NGC 2264

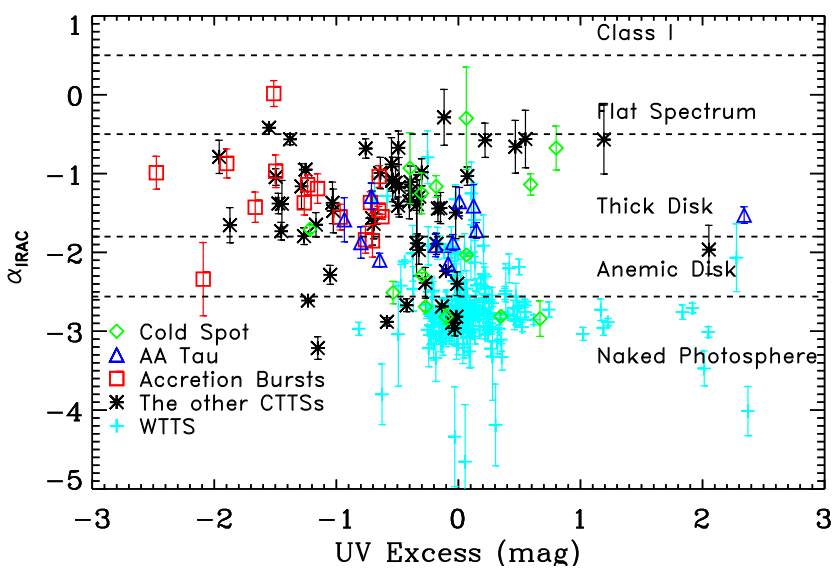

Fig. 8. Comparison between the CoRoT light-curve classification with the UV excess from Venuti et al. (2014) and the slope of the spectral energy distribution from $3.6 \mu \mathrm{m}$ to $8 \mu \mathrm{m}\left(\alpha_{\text {IRAC }}\right)$. These results show that the CoRoT light-curve morphology is related to the accretion process and the evolution of dust in the inner disk.

classes, but are mostly found among the anemic disks and naked photosphere populations. All AA Tau-like systems have dust in the inner disk, which is important, since we assumed their lightcurve variability to be dominated by circumstellar dust extinction from that region. Most of the accretion burst systems present thick disks, which is consistent with an earlier disk evolution and accretion phase than the AA Tau-like stars. The mean $\alpha_{\text {IRAC }}$ values of each light-curve group corroborate this analysis: -1.42 for the accretion bursts, -1.71 for the AA Tau systems, -1.84 for the spot-like systems, and -2.76 , for the WTTSs. These results show that the CoRoT light-curve morphology is related to the evolution of the inner disk. It also shows that accretion and disk evolution are related to each other, since the stars with highest UV excesses (more negative values) are the ones with the dustiest inner disks, while stars with little or no sign of accretion have almost no dust in their inner disks.

Figure 8 also shows some interesting objects that deserve further investigation, which beyond the scope of the present paper, however, such as the WTTSs among the anemic and thick disk systems. These are non-accreting stars (based on spectra) that still show substantial dust in their inner disks. In spite of this, for some as yet unknown reason, accretion has been shut off or is at a very low level in these systems, at least at the time of spectroscopic observation. There is also a significant number of anemic disk candidates among the CTTSs, with various light-curve morphologies that would be interesting targets to investigate the inner disk dispersal in accreting systems.

\section{Equivalent width and accretion rates from the $\mathrm{H} \alpha$ line}

The $\mathrm{H} \alpha$ line of a CTTS is generally quite strong and wide and is often characterized by its equivalent width. Compared to the morphology of the CoRoT light curves, the accretion burst systems show the highest $\mathrm{EW}_{\mathrm{H} \alpha}$ in our sample, with an average value of $96 \pm 19 \AA$, clearly distinguishable from the AA Tau and spot-like systems. AA Tau-like stars present an average $\mathrm{EW}_{\mathrm{H} \alpha}$ of $13.2 \pm 2.4 \AA$, while CTTSs with spot-like light curves present a mean $\mathrm{EW}_{\mathrm{H} \alpha}$ value of $21.1 \pm 5.6 \AA$. The uncertainties correspond to the standard deviations of the measured equivalent width values. The distribution of $\mathrm{EW}_{\mathrm{H} \alpha}$ among the three CTTS groups is shown in Fig. 9, where we included $\mathrm{EW}_{\mathrm{H} \alpha}$ measurements from

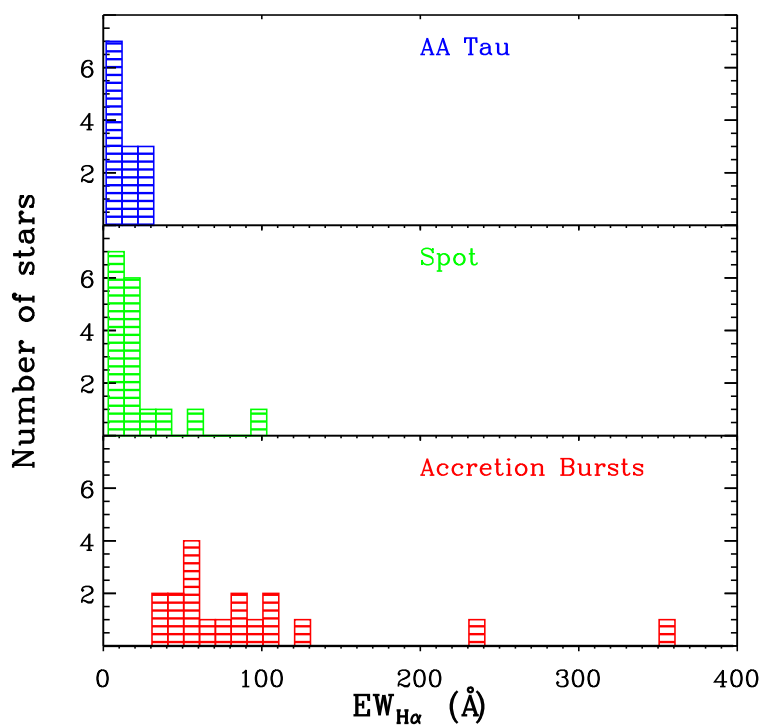

Fig. 9. Distribution of $\mathrm{H} \alpha$ equivalent width of CTTSs that present AA Tau-like (top), spot-like (middle), and accretion burst (bottom) light curves.

Dahm \& Simon (2005) for the stars that we did not observe. The same tendency is observed with the $\mathrm{H} \alpha$ width at $10 \%$ of maximum line intensity.

Mass-accretion rates $\left(\dot{M}_{\mathrm{H} \alpha}\right)$ are frequently calculated from the $\mathrm{H} \alpha$ line flux, using calibrations such as the ones proposed by Fang et al. (2009) and Gullbring et al. (1998). We compared the mass-accretion rates obtained from the UV excess by Venuti et al. (2014) with the values obtained with the $\mathrm{H} \alpha$ flux, as described below.

We calculated the $\mathrm{H} \alpha$ flux $\left(F_{\mathrm{H} \alpha}\right)$ from the $\mathrm{EW}_{\mathrm{H} \alpha}$,

$F_{\mathrm{H} \alpha}=E W_{\mathrm{H} \alpha} F_{\mathrm{c}}(\mathrm{H} \alpha)$

where $F_{\mathrm{c}}(\mathrm{H} \alpha)$ is the continuum flux in the $\mathrm{H} \alpha$ region. Since the FLAMES spectra were not flux calibrated, we estimated $F_{\mathrm{c}}(\mathrm{H} \alpha)$ from the CoRoT flux using CoRoT observations obtained simultaneously with the FLAMES spectra. The CoRoT flux was converted into $R$ magnitudes using the $R$-band photometry zeropoint of 26.74, as determined by Cody et al. (2014). For the stars observed with FLAMES that were not observed with CoRoT in 2011, we used the continuum flux from the CFHT $r$ band (Venuti et al. 2014) taken simultaneously with the FLAMES observations. The continuum flux was then corrected for extinction with $A_{V}$ obtained from the CFHT data (Venuti et al. 2014).

The $\mathrm{H} \alpha$ luminosity was then computed as $L_{\mathrm{H} \alpha}=4 \pi \mathrm{d}^{2} F_{\mathrm{H} \alpha}$, where we used $d=760 \mathrm{pc}$ (Sung et al. 1997) as the distance to the cluster. We used the fit proposed by Fang et al. (2009) to calculate the accretion luminosity $\left(L_{\mathrm{acc}}\right)$ from $L_{\mathrm{H} \alpha}$ :

$\log \left(\frac{L_{\mathrm{acc}}}{L_{\odot}}\right)=(2.27 \pm 0.23)+(1.25 \pm 0.07) \log \left(\frac{L_{\mathrm{H} \alpha}}{L_{\odot}}\right)$.

With the accretion luminosity, we obtained the mass-accretion rate,

$\dot{M}_{\mathrm{H} \alpha}=\frac{L_{\mathrm{acc}} R_{*}}{\mathrm{GM}_{*}\left(1-\frac{R_{*}}{R_{\mathrm{in}}}\right)}$,

where $R_{*}$ and $M_{*}$ are the radius and mass of the star, respectively, taken from Venuti et al. (2014), and G denotes the gravitational 


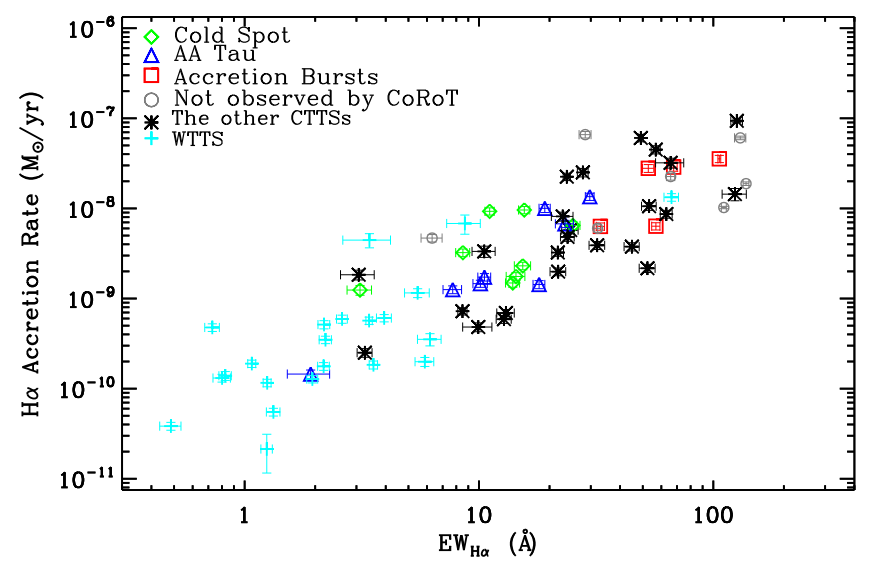

Fig. 10. Comparison between the CoRoT light-curve classification and mass-accretion rates obtained from $\mathrm{H} \alpha$ flux as a function of $\mathrm{H} \alpha$ equivalent width (FLAMES/VLT data). The error bars represent the night-tonight variability of the line flux.

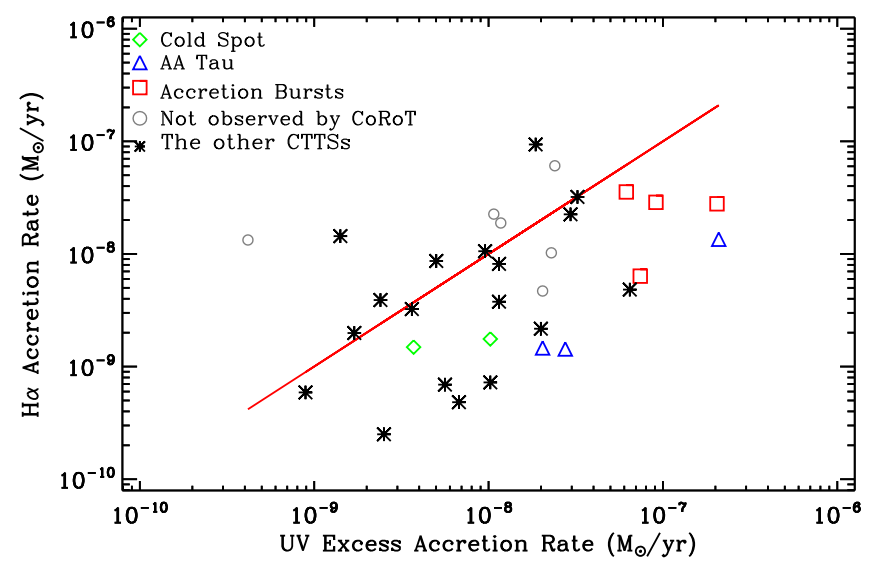

Fig. 11. Comparison between mass-accretion rates obtained from $\mathrm{H} \alpha$ flux with the UV excess mass-accretion rates. The red line shows a slope equal to 1 .

constant. The inner radius was set to $R_{\text {in }}=5 R_{*}$, which is a standard value of the disk truncation radius of CTTSs (Gullbring et al. 1998). The individual values of mass-accretion rates calculated from the $\mathrm{H} \alpha$ line flux are presented in Table 6.

Figure 10 shows the mass-accretion rates obtained from the mean $\mathrm{H} \alpha$ equivalent width of the four to six FLAMES and CoRoT simultaneous observations, and also those obtained from simultaneous CFHT observations. We plot the mean $\mathrm{EW}_{\mathrm{H} \alpha}$, and the error bar represents the night-to-night variability of $\mathrm{EW}_{\mathrm{H} \alpha}$ and $\dot{M}_{\mathrm{H} \alpha}$. As observed with the $\dot{M}_{\mathrm{UV}}$ relation (Fig. 7), stars that present accretion burst light curves have higher $\dot{M}_{\mathrm{H} \alpha}$. Since WTTSs are not actually accreting, their $\mathrm{H} \alpha$ equivalent widths are probably of chromospheric origin, a contribution that is also present in the CTTS spectra. Therefore, the mass-accretion rate locus of WTTSs represents a lower limit to our ability to measure mass-accretion rates of CTTSs from the $\mathrm{H} \alpha$ equivalent width in our sample, as discussed by Ingleby et al. (2011) and Manara et al. (2013).

Figure 11 shows the relation of the mass-accretion rates calculated with the UV excess and $\mathrm{H} \alpha$ equivalent width. The two methods show similar tendencies, but the individual value can sometimes be different. This difference may arise because the $\mathrm{H} \alpha$ line does not necessarily originate in the accretion columns alone, but can also have contributions from the disk wind and accretion shock, as absorptions superposed on the accretion profile.
When we calculated the $\mathrm{H} \alpha$ equivalent width, the absorptions decrease the measured $\mathrm{EW}_{\mathrm{H} \alpha}$ value, leading to lower values of mass-accretion rates. At the same time, the errors in $A_{\mathrm{V}}$ affect the UV excess accretion rates more than the $\mathrm{H} \alpha$ ones and can lead to more uncertain values of mass-accretion rates calculated from UV excess. Although UV excess is clearly a more direct measurement of the mass-accretion rate, $\mathrm{H} \alpha$ line fluxes are much easier to obtain and allow for a good estimate of mass-accretion rates for all accreting objects, even those that do not present a substantial UV excess, due, for example, to circumstellar dust obscuration of the hot spot.

\section{7. $\mathrm{H} \alpha$ line variability}

The CTTSs in NGC 2264 present a variety of $\mathrm{H} \alpha$ line profiles. In this section, we verify how common each $\mathrm{H} \alpha$ profile type is and compare its occurrence with the CoRoT light-curve classification. We followed the $\mathrm{H} \alpha$ profile classification proposed by Reipurth et al. (1996), which is divided into seven profile types. A type I profile is symmetric and shows no absorption feature. A type II profile presents two peaks, and the less intense one exceeds half the intensity of the main peak. A type III profile also shows two peaks, and the less intense one is smaller than half the intensity of the main peak. A type IV profile shows a pronounced absorption feature on the blue or red side of the line where no emission is seen, like a P Cygni or inverse P Cygni profile. The letters B or R are attached to types II, III and IV, depending on the location (in the blue (B) or red (R) side of the line) of the secondary peak or absorption feature with respect to the main peak. We have 20 to 22 nights of observation of each target, and the $\mathrm{H} \alpha$ line is quite variable both in intensity and shape from night to night. Therefore, we morphologically classified only the mean $\mathrm{H} \alpha$ profile of each star and present this classification in Table 6 and Fig. 12. The top panel of Fig. 12 shows the profile distribution of all CTTSs observed with FLAMES. Type I is the most common $\mathrm{H} \alpha$ profile in our sample. This profile corresponds to a main emission feature without any prominent absorption and is attributed to magnetospheric accretion. The emission profiles of type I stars sometimes display some superposed absorption in one or a few observing nights, but the analyzed mean profile effectively does not present absorptions.

Figure 12 (bottom) shows the distribution of the $\mathrm{H} \alpha$ profile types according to the CoRoT light-curve classification. Spotlike stars typically present type I $\mathrm{H} \alpha$ profiles, generally showing no evidence of blueshifted absorption due to wind, or redshifted absorption due to hot spot photons absorbed by the accretion funnel. Moreover, AA Tau-like stars tend to present type IIR and IIIR profiles, which are characterized by redshifted absorptions, compared to the most common type I profile seen among the CTTSs in our sample.

Kurosawa et al. (2006) used disk-wind and magnetosphere radiative transfer models for CTTS to examine the $\mathrm{H} \alpha$ classification proposed by Reipurth et al. (1996) and with a range of parameters reproduced all the $\mathrm{H} \alpha$ profile types. In general, they found that the type I profile is the most common among CTTSs, since it is produced from a range of system inclinations and is dominated by magnetospheric emission. Kurosawa et al. (2006) found that type IIB and IIR profiles were equally common, as also found by Reipurth et al. (1996) and by us, since they result from the same physical conditions, with the exception of the system inclination. Type IIB needs medium inclinations to be produced, while type IIR results from high inclinations. This is also consistent with our results, since most AA Tau-like stars, 
A. P. Sousa et al.: CSI 2264: Accretion process in classical T Tauri stars in the young cluster NGC 2264

Table 6. Periods of the classical T Tauri stars observed with the FLAMES spectrograph obtained with photometric and spectroscopic diagnostics.

\begin{tabular}{|c|c|c|c|c|c|c|c|c|c|}
\hline Mon $\mathrm{ID}^{a}$ & LC_2008 ${ }^{b}$ & 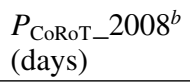 & LC_2011 ${ }^{b}$ & $\begin{array}{l}P_{\text {CoRoT_} \_2011^{b}}^{b} \\
\text { (days) }\end{array}$ & $\begin{array}{l}P_{\mathrm{H}{ }^{c}} \\
\text { (days) }\end{array}$ & $\begin{array}{l}P_{\mathrm{HeI}}^{d} \\
\text { (days) }\end{array}$ & Regime $^{e}$ & Н $\alpha$ Type $^{f}$ & $\begin{array}{l}\dot{M}_{\mathrm{H} \alpha}{ }^{g} \\
\left(M_{\odot} / \mathrm{yr}\right)\end{array}$ \\
\hline CSIMon-001099 & 1 & 3.31 & 1 & 3.38 & NP & - & $\mathrm{S}$ & IIB & $3.23 \mathrm{e}-09$ \\
\hline CSIMon-000103 & 1 & 1.67 & 1 & 3.35 & NP & - & & I & $9.61 \mathrm{e}-09$ \\
\hline CSIMon-000177 & 1 & 3.01 & 1 & 3.01 & NP & - & & I & $9.29 \mathrm{e}-09$ \\
\hline CSIMon-000335 & 1 & 4.51 & 1 & 4.58 & NP & - & & I & $2.31 \mathrm{e}-09$ \\
\hline CSIMon-000965 & 1 & 9.38 & 1 & 9.68 & NP & 9.4 & $\mathrm{~S}$ & IIR & $1.76 \mathrm{e}-09$ \\
\hline CSIMon-000810 & 1 & 2.93 & 1 & 2.93 & NP & - & & I & $1.24 \mathrm{e}-09$ \\
\hline CSIMon-000964 & 1 & 3.34 & 1 & 3.32 & NP & - & & I & $1.49 \mathrm{e}-09$ \\
\hline CSIMon-000326 & 1 & 7.05 & 3 & & NP & NP & & I & $1.99 \mathrm{e}-09$ \\
\hline CSIMon-000220 & 1 & 0.76 & 3 & & NP & - & & I & $2.50 \mathrm{e}-10$ \\
\hline CSIMon-000804 & 1 & 3.23 & 3 & & NP & - & & I & $4.83 e-10$ \\
\hline CSIMon-001033 & 1 & 14.15 & - & & 6.30 & - & S & IIR & $4.69 \mathrm{e}-09$ \\
\hline CSIMon-000250 & 2 & 4.16 & 2 & 8.93 & $7.5 / 8.9$ & 8.6 & S & IIR & $1.44 \mathrm{e}-09$ \\
\hline CSIMon-000660 & 2 & 5.25 & 2 & 5.10 & 5.2 & - & S & IIIR & $1.45 \mathrm{e}-10$ \\
\hline CSIMon-000498 & 2 & 4.23 & 2 & 4.28 & NP & - & & I & $1.00 \mathrm{e}-08$ \\
\hline CSIMon-001199 & 2 & 3.75 & 2 & 3.61 & NP & - & & IIB & $1.46 \mathrm{e}-09$ \\
\hline CSIMon-000297 & 2 & 3.16 & $3(\mathrm{E})$ & & NP & - & & IIR & $5.75 \mathrm{e}-09$ \\
\hline CSIMon-000928 & 2 & 4.96 & 3 & & NP & 10.3 & & I & $6.91 \mathrm{e}-10$ \\
\hline CSIMon-000654 & 2 & 4.66 & $3(\mathrm{E})$ & & NP & NP & & I & $4.82 \mathrm{e}-09$ \\
\hline CSIMon-000441 & 2 & 4.06 & $3(\mathrm{E})$ & & NP & Noise & & IIB & $3.24 \mathrm{e}-09$ \\
\hline CSIMon-000824 & 2 & 7.05 & - & & NP & - & $\mathrm{U}$ & IVR & $3.28 \mathrm{e}-09$ \\
\hline CSIMon-001144 & $3(\mathrm{E})$ & & $3(\mathrm{E})$ & & NP & Noise & & II & $2.17 \mathrm{e}-09$ \\
\hline CSIMon-001275 & 3 & & 3 & & NP & - & & IIIB & $2.51 \mathrm{e}-08$ \\
\hline CSIMon-000119 & 3 & & 3 & & NP & NP & & IIIB & $3.34 \mathrm{e}-09$ \\
\hline CSIMon-000558 & $3(\mathrm{~A})$ & & 3 & $11.70 ?$ & 10.5 & 10.7 & & IIB & $6.06 \mathrm{e}-08$ \\
\hline CSIMon-000168 & $3(\mathrm{E})$ & & $3(\mathrm{E})$ & & $\mathrm{NP}$ & Noise & & II & $3.77 \mathrm{e}-09$ \\
\hline CSIMon-000926 & 3 & & 3 & & NP & NP & & I & $8.66 \mathrm{e}-09$ \\
\hline CSIMon-000681 & $3(\mathrm{E})$ & & $3(\mathrm{E})$ & & NP & NP & & IIB & $4.49 \mathrm{e}-08$ \\
\hline CSIMon-000280 & 3 & & 3 & & NP & - & & IIR & $5.90 \mathrm{e}-10$ \\
\hline CSIMon-000290 & 3 & & 3 & & NP & $8.3 / 11.4$ & & I & $2.25 \mathrm{e}-08$ \\
\hline CSIMon-000328 & 3 & & 3 & & NP & NP & & I & $3.90 \mathrm{e}-09$ \\
\hline CSIMon-000448 & 3 & & 3 & & NP & Noise & & I & $8.16 \mathrm{e}-09$ \\
\hline CSIMon-000314 & $3(\mathrm{E})$ & & $3(\mathrm{E})$ & & NP & Noise & & I & $1.06 \mathrm{e}-08$ \\
\hline CSIMon-000951 & 3 & & 3 & & NP & Noise & & I & $3.21 \mathrm{e}-08$ \\
\hline CSIMon-000937 & 3 & & 3 & & NP & - & & I & $7.24 \mathrm{e}-10$ \\
\hline CSIMon-000667 & $3(\mathrm{E})$ & & $3(\mathrm{E})$ & & 5.9 & - & S & IIB & $1.84 \mathrm{e}-09$ \\
\hline CSIMon-000945 & $3(\mathrm{~A})$ & & $3(\mathrm{~A})$ & & NP & 7.4 & & I & $6.35 \mathrm{e}-09$ \\
\hline CSIMon-001022 & $3(\mathrm{~A})$ & & $3(\mathrm{~A})$ & & NP & $8.9 / 12.5$ & & IIIB & $6.31 \mathrm{e}-09$ \\
\hline CSIMon-000996 & $3(\mathrm{~A})$ & & $3(\mathrm{~A})$ & & 8.3 & 8.3 & & I & $2.79 \mathrm{e}-08$ \\
\hline CSIMon-000510 & $3(\mathrm{~A})$ & & $3(\mathrm{~A})$ & & NP & NP & & I & $2.87 \mathrm{e}-08$ \\
\hline CSIMon-000341 & $3(\mathrm{~A})$ & & $3(\mathrm{~A})$ & & NP & NP & & I & $3.55 \mathrm{e}-08$ \\
\hline CSIMon-000370 & 3 & & 3 & $11.82 ?$ & NP & NP & & I & $2.55 \mathrm{e}-08$ \\
\hline CSIMon-000765 & 3 & & 1 & 2.41 & NP & - & & I & $6.48 \mathrm{e}-09$ \\
\hline CSIMon-000379 & $3(\mathrm{E})$ & & 2 & 3.68 & NP & - & $\mathrm{S}$ & IIR & $1.26 \mathrm{e}-09$ \\
\hline CSIMon-001054 & $3(\mathrm{E})$ & & 2 & $4.08 / 8.17 *$ & NP & 8.1 & & I & $1.35 \mathrm{e}-08$ \\
\hline CSIMon-000296 & $3(\mathrm{E})$ & & 2 & 3.91 & NP & - & $\mathrm{S}$ & IIR & $1.73 \mathrm{e}-09$ \\
\hline CSIMon-000811 & $3(\mathrm{E})$ & & 2 & 7.88 & 12.5 & 10.5 & S & IIR & $6.75 \mathrm{e}-09$ \\
\hline CSIMon-000846 & 3 & & - & & NP & NP & & I & - \\
\hline CSIMon-000795 & 3 & & - & & NP & NP & & IIB & $6.59 \mathrm{e}-08$ \\
\hline CSIMon-000457 & 3 & & - & & NP & - & & IIIB & $6.01 \mathrm{e}-09$ \\
\hline CSIMon-000260 & $3(\mathrm{E})$ & & - & & NP & 9.2 & & IIB & $2.26 \mathrm{e}-08$ \\
\hline CSIMon-000893 & 3 & & - & & NP & NP & & I & $1.02 \mathrm{e}-08$ \\
\hline CSIMon-000131 & - & & 3 & & NP & 9.1 & & IIR & - \\
\hline CSIMon-000423 & - & & 3 & & NP & Noise & & I & $1.44 \mathrm{e}-08$ \\
\hline CSIMon-000632 & - & & - & & NP & Noise & & IIB & $1.33 \mathrm{e}-08$ \\
\hline CSIMon-001287 & - & & - & & NP & Noise & & IIR & $9.38 \mathrm{e}-08$ \\
\hline CSIMon-000603 & - & & - & & NP & Noise & & IIIB & $6.07 \mathrm{e}-08$ \\
\hline CSIMon-000239 & - & & - & & 5.6 & - & & $?$ & - \\
\hline CSIMon-001128 & - & & - & & NP & Noise & & I & $1.88 \mathrm{e}-08$ \\
\hline
\end{tabular}

Notes. This table is ordered by the classification of the CoRoT light-curve morphology from the 2008 campaign. ${ }^{(a)}$ CSIMon is an internal identification of the CSI 2264 campaign. ${ }^{(b)}$ CoRoT light-curve morphology and photometric period obtained in this work: $1=$ spot-like, $2=$ AA Tau-like, 3 = non-periodic light curves. Accretion bursts stars and aperiodic extinction light curves are identified by (A) and (E), respectively. (c) Period obtained with the $\mathrm{H} \alpha$ line from FLAMES spectra. NP denotes a non-periodic line. ${ }^{(d)}$ Period obtained with HeI line $6678 \AA$ from FLAMES spectra. Noise means that the HeI line is present on the spectrum but it is dominated by noise. ${ }^{(e)}$ Accretion regime: S-stable and Uunstable, as discussed in Sect. 10. ${ }^{(f)}$ Morphology of $\mathrm{H} \alpha$ line by Reipurth et al. (1996) discussed in Sect. 7. ${ }^{(g)}$ Mass-accretion rates calculated with $\mathrm{H} \alpha$ equivalent width. ${ }^{(*)}$ The photometric period was obtained using Scargle periodogram and auto-correlation function, as explained in Sect. 9.1. 

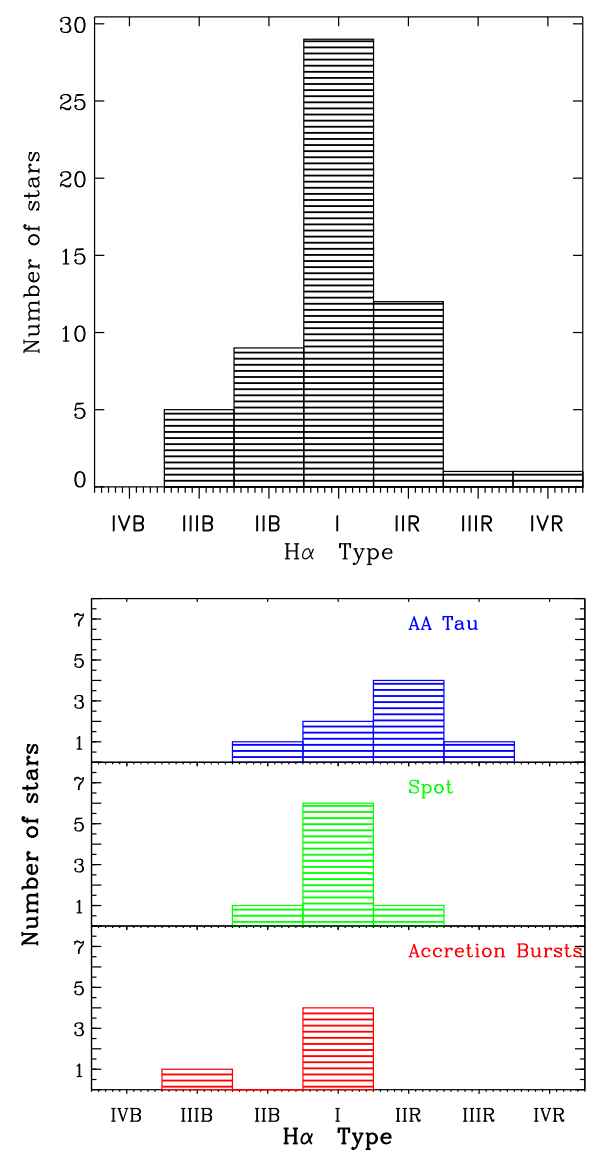

Fig. 12. Distribution of $\mathrm{H} \alpha$ profile types according to the classification of Reipurth et al. (1996). Top: all the CTTSs observed with FLAMES. Bottom: CTTSs observed both with FLAMES and CoRoT. Light curves classified as spot-like, AA Tau-like, and accretion burst are shown as a function of profile type.

which are high-inclination systems (McGinnis et al. 2015), tend to present type IIR profiles (see Fig. 12, bottom).

Reipurth et al. (1996) instead found that the $\mathrm{H} \alpha$ profile distribution in their sample was dominated by type IIIB profiles (33\% of their sample), while in our sample only $\sim 9 \%$ of the observed CTTSs present type IIIB profiles. In the models of Kurosawa et al. (2006), only high mass-accretion rate stars presented type IIIB profiles. The difference in profile distribution found by us and Reipurth et al. (1996) might therefore simply be due to a sample selection effect. The stars classified as type IIIB by Reipurth et al. (1996) have spectral types between $\mathrm{G} 0$ and $\mathrm{K} 7$ and a very high $\mathrm{H} \alpha$ equivalent width (average value of $64 \AA$ ), which may indeed indicate that they are high mass-accretion rate systems. The type IIIB CTTSs in our sample present an average EW of $47 \AA$ and a mean mass-accretion rate of $\sim 2.5 \times 10^{-8} M_{\odot} / \mathrm{yr}$, which is not particularly high, but does correspond to one of the highest mean mass-accretion rates of the various profile types.

\section{Periodograms of $\mathrm{H} \alpha$ emission line}

As the star-disk system rotates, the observed $\mathrm{H} \alpha$ line of CTTSs varies in intensity and shape. This is expected to occur, even in a stable accretion regime, if the stellar magnetic and rotation axis are not aligned. The misalignment between the axes breaks the axisymmetry of the accretion environment and creates individual accretion funnels in each hemisphere. The projection of the $\mathrm{H} \alpha$ emission region in our line of sight will then be different at every rotational phase, which will cause line variability (see for example Alencar et al. 2012). If most of the $\mathrm{H} \alpha$ line is formed in the accretion columns, we would expect to measure a periodic line variability when the star is in a stable accretion regime. However, the $\mathrm{H} \alpha$ line may also form in different circumstellar regions, such as the chromosphere, disk wind, and jet, and each region may have a different periodicity. This would make a single period difficult to detect. With 20 to 22 nights of observation of each target with the FLAMES spectrograph, we can check whether the $\mathrm{H} \alpha$ line variations were periodic, and compare these periods with the periodicities measured photometrically. This will allow us to infer if the $\mathrm{H} \alpha$ line-forming region is directly related to the physical process causing the photometric variability.

We analyzed the line periodicity by calculating Scargle periodograms, as modified by Horne \& Baliunas (1986), along the line profile. The Scargle periodograms fit the data with sinusoidal functions through a least-squares method. The code requires a range of periods to be tested, and the best fit corresponds to the highest power in the periodogram. To obtain the periodicity in the $\mathrm{H} \alpha$ line, we divided each normalized $\mathrm{H} \alpha$ line profile into small velocity intervals $\left(0.5 \mathrm{~km} \mathrm{~s}^{-1}\right)$. For each velocity interval, Scargle periodograms were independently calculated. Each periodogram was grouped side by side in velocity, to form a diagram of period vs. velocity. The power of the periodogram is represented by colors, varying from zero (white) to the maximum power intensity (black). With this procedure, we can check how each period spreads across the $\mathrm{H} \alpha$ line. Periodic signals only in a few velocity bins may not be significant, but periodic signals that are spread across several velocity channels indicate periodicity in an extended region of line formation. We can also verify if there are different periodicities in different line regions (like in emission and absorption).

Only eight CTTSs observed with the FLAMES spectrograph presented a periodic signal in the $\mathrm{H} \alpha$ line (see Table 6), and their periodograms are shown in Figs. 13-15. These figures are discussed individually in the following subsections, where we show that most of the periodicity in the $\mathrm{H} \alpha$ line occurs in the red wing, which comes from gas moving away from the observer and probably is related to the accretion process. These periodic stars presented organized and stable accretion regions rotationally modulated by the stellar magnetic field.

Our spectroscopic target selection in 2011 was largely based on the CoRoT light-curve classification from 2008, and we included as many AA Tau-like and spot-like systems as possible in our two pointings to study periodic systems. We did not anticipate, however, that a large number of systems would change their light-curve variability from periodic to aperiodic between the two runs. Of the 58 stars observed with FLAMES, only 16 presented periodic CoRoT light curves in 2011,8 of which were classified as AA Tau-like and the other 8 as spot-like systems. Probably because of our unfortunate selection, the $\mathrm{H} \alpha$ line of most of the 58 observed CTTSs showed no periodicity in our data.

In the next subsections, we analyze the $\mathrm{H} \alpha$ periodicity compared to the CoRoT light-curve morphology discussed in Sect. 4. Because of the large number of stars observed spectroscopically with FLAMES that presented a non-periodic CoRoT light curve in 2011, our main conclusion is that the stars that presented nonperiodic CoRoT light curves are generally not periodic in $\mathrm{H} \alpha$ either. We show in Sect. 10 that the lack of periodicity in $\mathrm{H} \alpha$ and in the photometry may be caused by an unstable accretion regime, as proposed by Kurosawa \& Romanova (2013). 


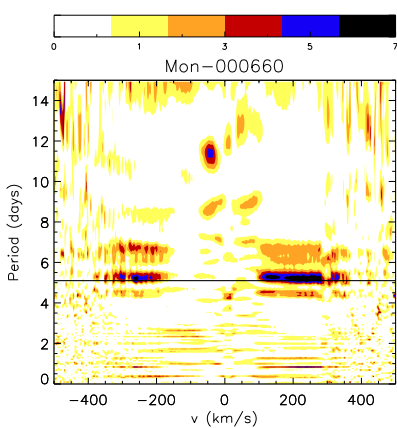

(a)

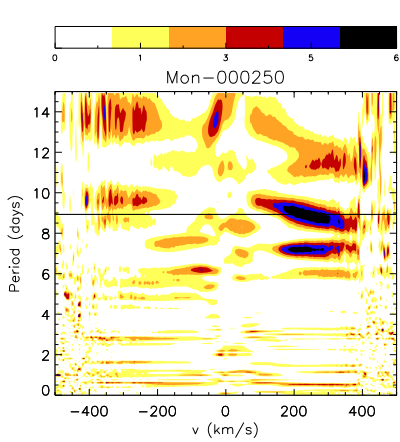

(d)

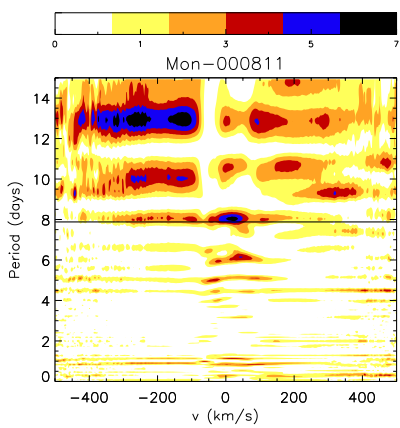

(g)

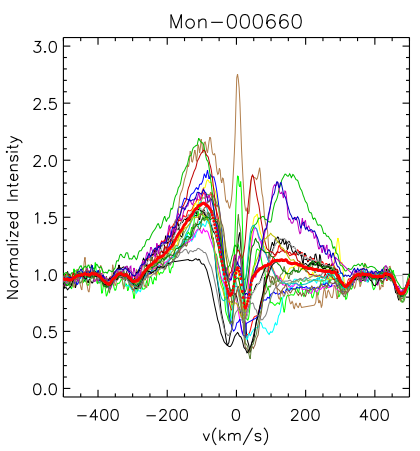

(b)

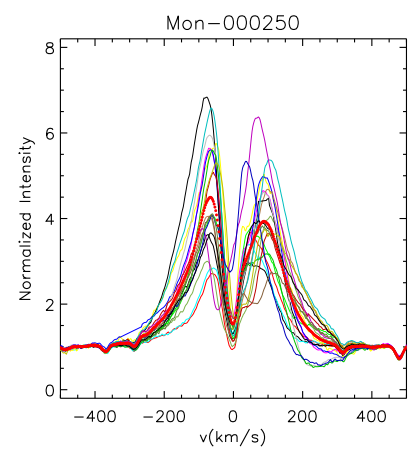

(e)

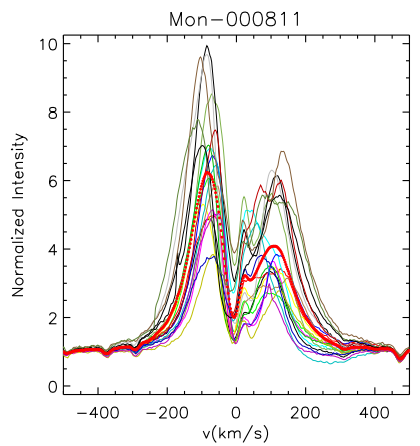

(h)

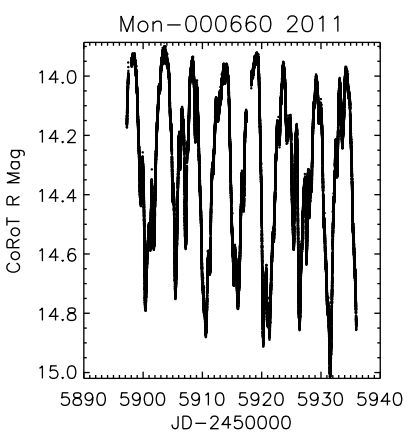

(c)

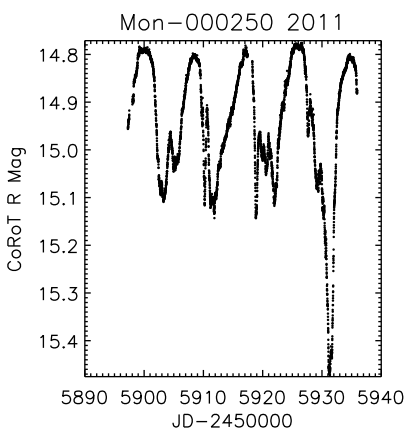

(f)

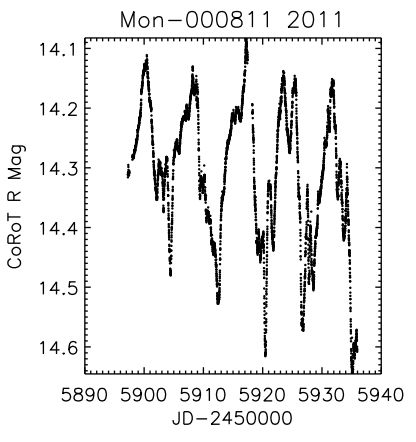

(i)

Fig. 13. AA Tau-like stars in 2011 that present a periodicity in the $\mathrm{H} \alpha$ line. Left column: bidimensional periodograms of the $\mathrm{H} \alpha$ line. The color code represents the power of periodogram, varying from zero (white) to the maximum power intensity (black). The black horizontal lines correspond to the photometric period of the 2011 CoRoT light curves. Middle column: $\mathrm{H} \alpha$ line profiles. Different colors correspond to different observation nights, and the thick red line is the average line profile. Right column: CoRoT light curves from 2011.

Table 7. Parameters used to calculate corotation radius $\left(R_{\mathrm{C}}\right)$ and magnetic field strength $\left(B_{*}\right)$ of the three AA Tau-like stars that present observational evidence that the inner disk warp is located at the corotation radius.

\begin{tabular}{llllllll}
\hline \hline Mon ID $^{a}$ & $\begin{array}{l}M_{*}{ }^{b} \\
\left(M_{\odot}\right)\end{array}$ & $\begin{array}{l}R_{*}{ }^{c} \\
\left(R_{\odot}\right)\end{array}$ & $\begin{array}{l}\dot{M}_{\mathrm{H} \alpha}{ }^{d} \\
\left(M_{\odot} / \mathrm{yr}\right)\end{array}$ & $\begin{array}{l}P_{\mathrm{CoRoT} \_} 2011^{e} \\
(\text { days })\end{array}$ & $\begin{array}{l}P_{\mathrm{H} \alpha}{ }^{f} \\
(\text { days })\end{array}$ & $\begin{array}{l}R_{\mathrm{C}}{ }^{g} \\
\left(R_{*}\right)\end{array}$ & $\begin{array}{l}B_{*}{ }^{h} \\
(\mathrm{kG})\end{array}$ \\
\hline CSIMon-000250 & 1.63 & 1.35 & $1.44 \mathrm{e}-09$ & 8.93 & $7.5 / 8.9$ & 12.3 & 0.81 \\
CSIMon-000660 & 1.86 & 1.4 & $1.45 \mathrm{e}-10$ & 5.10 & 5.2 & 7.5 & 0.07 \\
CSIMon-000811 & 0.91 & 1.97 & $6.75 \mathrm{e}-09$ & 7.88 & $7.9 / 12.5^{*}$ & 8.2 & 0.44 \\
\hline
\end{tabular}

Notes. ${ }^{(a)}$ CSIMon is an internal identification of the CSI 2264 campaign. ${ }^{(b)}$ Stellar mass obtained by Venuti et al. (2014). ${ }^{(c)}$ Stellar radius obtained by Venuti et al. (2014). ${ }^{(d)}$ Mass-accretion rates calculated with $\mathrm{H} \alpha$ equivalent width. ${ }^{(e)}$ Photometric period obtained from the $2011 \mathrm{CoRoT}$ light curve. ${ }^{(f)}$ Period obtained with the $\mathrm{H} \alpha$ line from FLAMES spectra. ${ }^{(g)}$ Calculated corotation radius. ${ }^{(h)}$ Calculated stellar magnetic field. ${ }^{(*)}$ This star shows only a faint hint of periodicity in $\mathrm{H} \alpha$ at the photometric period ( $\sim 7.9$ days) that we used as the stellar rotational period.

\subsection{Ho periodicity in the AA Tau-like stars}

AA Tau-like stars are expected to be in a stable accretion regime, where a major funnel flow is formed in each hemisphere, and the base is associated with an inner disk warp that periodically occults the star as the system rotates (Bouvier et al. 2007a; Kurosawa et al. 2008). The photometric period of AA Taulike stars corresponds to the Keplerian period of the occulting 


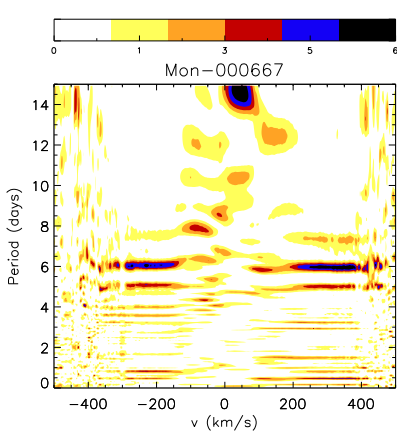

(a)

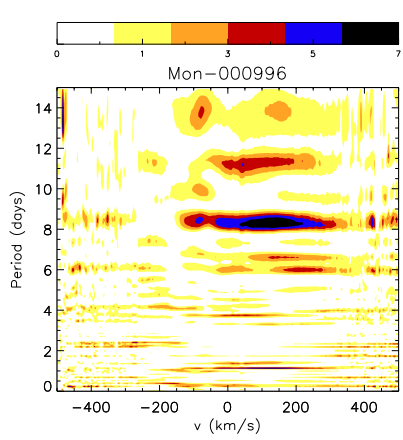

(d)

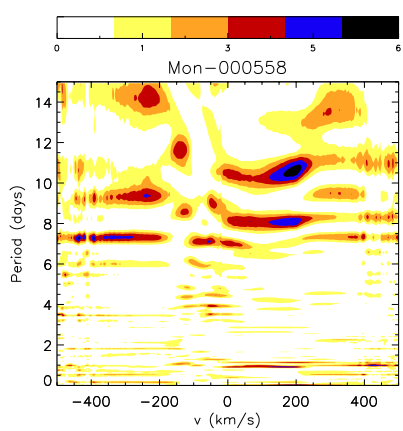

(g)

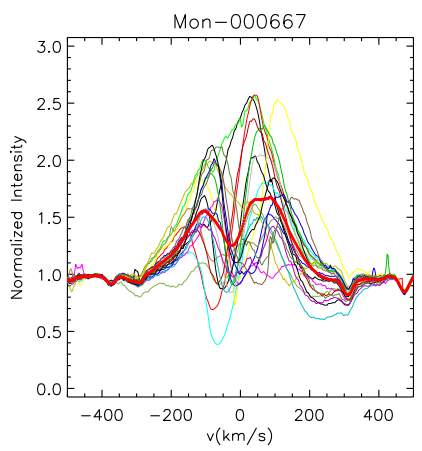

(b)

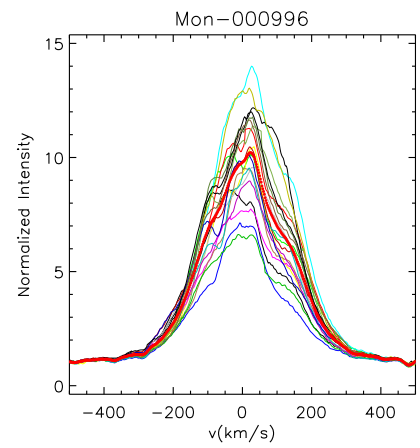

(e)

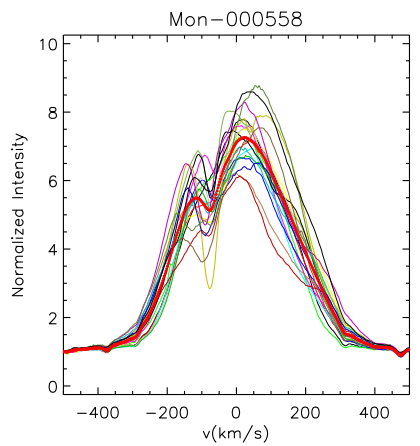

(h)

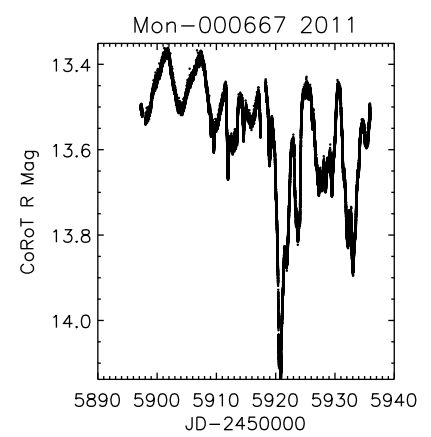

(c)

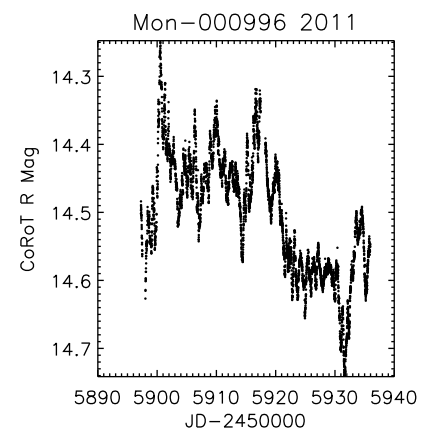

(f)

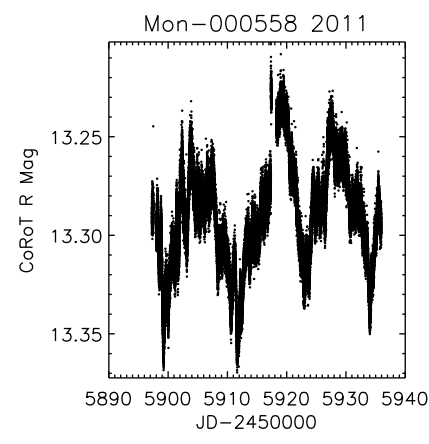

(i)

Fig. 14. Periodic stars in the $\mathrm{H} \alpha$ line that are not periodic in the CoRoT photometry. Left column: bidimensional periodograms of the H $\alpha$ line. The color code represents the power of periodogram, varying from zero (white) to the maximum power intensity (black). Middle column: H $\alpha$ line profiles. Different colors correspond to different nights of observation, and the thick red line is the average line profile. Right column: CoRoT light curves from the 2011 campaign.

circumstellar material, and it can be different from the stellar rotation period, unless the structure responsible for the occultation of the star is at the disk corotation radius. As shown in Sect. 4.2, at least in our sample, the photometric periods of AA Tau-like systems is not expected to differ substantially from the stellar rotation period, indicating that the inner disk warp is indeed located close to the disk corotation radius. Eight AA Tau-like stars were observed with the FLAMES spectrograph, but only three are periodic in $\mathrm{H} \alpha$ (see Fig. 13). This is somewhat unexpected. If the eight stars were in a stable accretion regime, it would naturally produce periodic variability in $\mathrm{H} \alpha$ if the line were mostly formed in the accretion funnel. However, if the accretion flow is significantly time variable, it could mask the periodicity imposed by the stellar rotation. We will explore evidence for the time variability of accretion during funnel-flow accretion in a forthcoming paper (Stauffer et al., in prep.).
The star Mon-000660 (V354 Mon), Figs. 13a-c, is an AA Tau-like analog that presents the same period in $\mathrm{H} \alpha$ and the photometry. Assuming that $\mathrm{H} \alpha$ is formed mainly in the accretion funnel and that the measured period corresponds to the stellar rotational period, it confirms that the occulting disk structure, which causes the photometric variability, is located at the corotation radius, as in AA Tau itself (Bouvier et al. 2007a). Another AA Tau-like star, Mon-000250, Figs. 13d-f, is also periodic in $\mathrm{H} \alpha$ and presents the same period in the photometry. In the $\mathrm{H} \alpha$ periodogram, this star shows two periodic signals in the red wing, the stronger power peak coincident with the photometric period ( $\sim 8.9$ days) and the weaker peak around $\sim 7.5$ days. This second period is also detected in the CoRoT light curve, but with a lower power than the main periodic signal, and is due to the periodicity of a small structure present inside the deepest dips of the light curve (see Fig. 13f). 


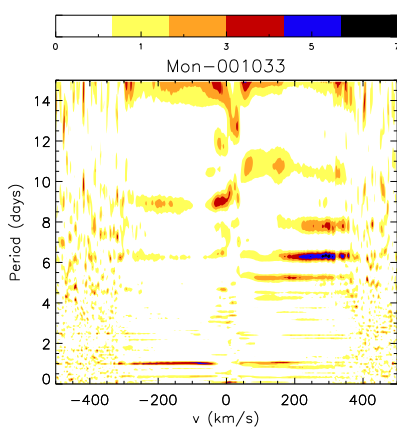

(a)

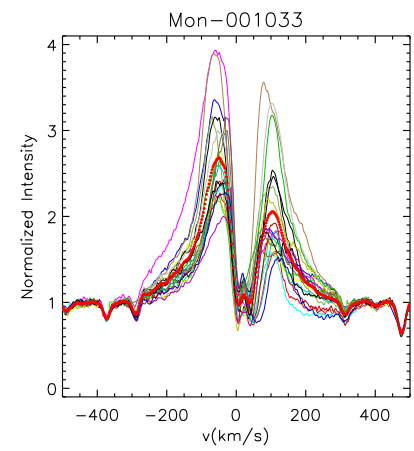

(b)

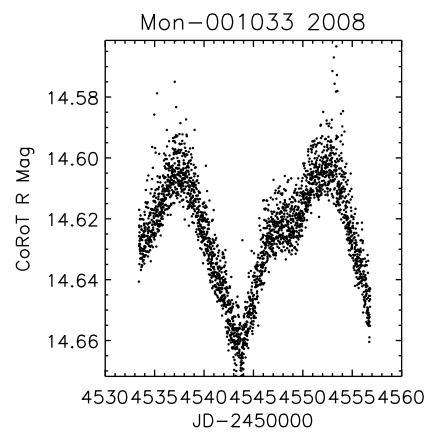

(c)

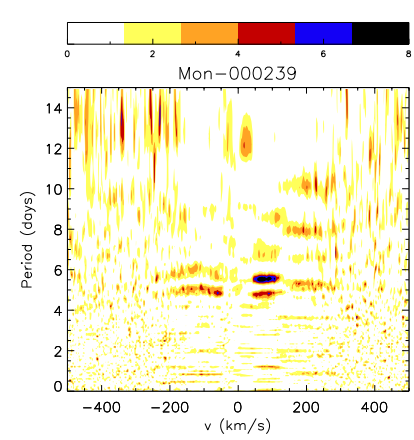

(d)

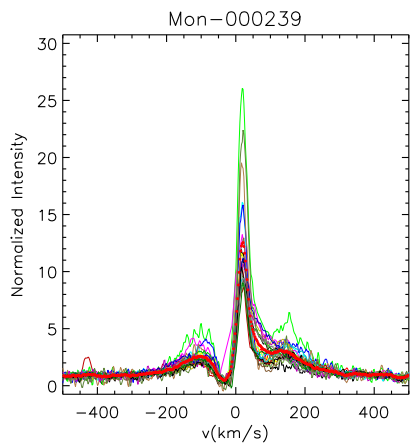

(e)

Fig. 15. Periodic stars in the $\mathrm{H} \alpha$ line that were not observed by CoRoT in 2011. Left column: bidimensional periodograms of the H $\alpha$ line. The color code represents the power of the periodogram, varying from zero (white) to the maximum power intensity (black). Middle column: H $\alpha$ line profiles. Different colors correspond to different nights of observation, and the thick red line is the average line profile. Right column: CoRoT light curve from 2008, when available.

On the other hand, star Mon-000811, Figs. 13g-i, which is also AA Tau-like, only shows a faint hint of periodicity in $\mathrm{H} \alpha$ at the photometric period ( $\sim 7.9$ days), and presents a strong periodic detection at about 13 days in the $\mathrm{H} \alpha$ blue wing. This star also presents two periodic signals in the HeI $6678 \AA$ line, one at the photometric period and another at about 11 days, as shown in Sect. 9. The HeI line is expected to be formed near the hot spot, close to the stellar photosphere, so one of its periodicities is probably close to the stellar rotation period. If the approximately eight-day period of HeI corresponds to the stellar rotational period, the structure that occults the star could be located close to the corotation radius of the star-disk system. This is the only star that presents a periodicity only in the blue wing of the $\mathrm{H} \alpha$ line. Mon-000811 can be compared to the CTTS SU Aur, which exhibited a blueshifted absorption in $\mathrm{H} \alpha$ that is periodic (Johns \& Basri 1994), and thought to be due to a wind (Giampapa et al. 1993). Its variability is explained as rotational modulation of the wind by the magnetic field of SU Aur. Mon-000811 shows no absorption in the blue wing of $\mathrm{H} \alpha$ (as seen in Fig. 13h), and we cannot easily associate the observed periodicity in the blue wing with a wind. However, the blue-wing period is longer than the HeI period (which we associate with the rotation period), so the material responsible for the $\mathrm{H} \alpha$ blue-wing periodicity is probably located outside the corotation radius and could indeed come from a disk wind.

As discussed above, three AA Tau-like stars present the same periodicity in the photometric and spectroscopic analysis. Since the spectroscopic periods most likely correspond to the stellar rotational periods, we can assume that, at least in these three cases, the inner disk warp is located at the corotation radius. Following Bessolaz et al. (2008), we computed the star-disk corotation radius and calculated the magnetic field strength needed to enforce disk truncation at the corotation radius. The results are presented in Table 7. At a few stellar radii from the star, the star-disk interaction is expected to occur mostly through the dipole component of the stellar magnetic field, and the calculated magnetic fields in Table 7 are within the range of values of the few dipole components measured in CTTSs $\left(B_{\text {dip }}=0.02 \mathrm{kG}\right.$ to $1.9 \mathrm{kG}$; see Table 2 from Gregory et al. 2012).

\subsection{H $\alpha$ periodicity in stars classified as non-periodic with the CoRoT photometry}

The stars Mon-000667 (random occultation by circumstellar material), Mon-000996 (accretion burst), and Mon-000558 (irregular variability, but not associated with a main physical process) showed no well-defined periodicity in their CoRoT light curves in 2008 and in 2011, as shown in Table 6. These stars are periodic in $\mathrm{H} \alpha$, however, as seen in Figs. 14a, d, and g.

Mon-000667 has a period of 5.92 days measured by Lamm et al. (2004), which is very similar to our detected period ( $\sim 5.9$ days) and could indeed correspond to the stellar rotational period. In this case, the accreting gas is apparently organized, but the inner dust environment does not show a clear correlation with the gas structure.

We could not find any information about the rotational period of Mon-000996 in the literature, but it presents an $\mathrm{H} \alpha$ period ( $\sim 8.3$ days) that is equal to the period found in the HeI $6678 \AA$ line (see also Fig. 180; we discuss this in Sect. 9). This is quite unexpected, since its light curve is dominated by random hot spot variability, which would suggest unstable accretion and 

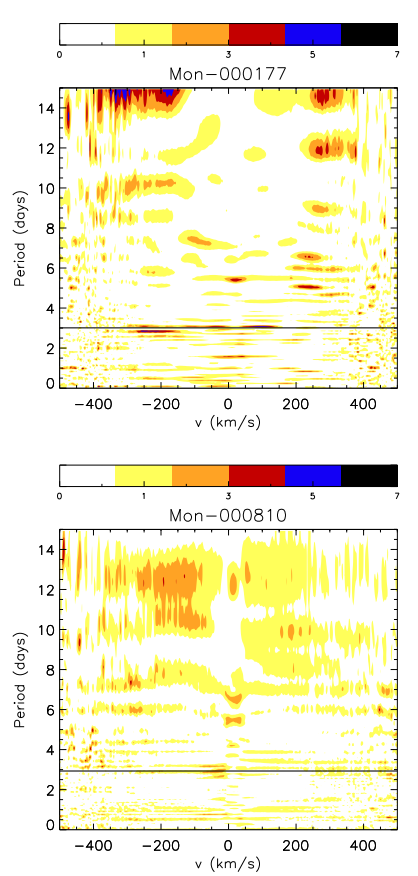

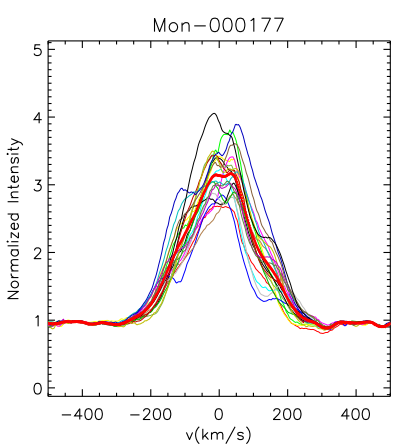

Mon-000810

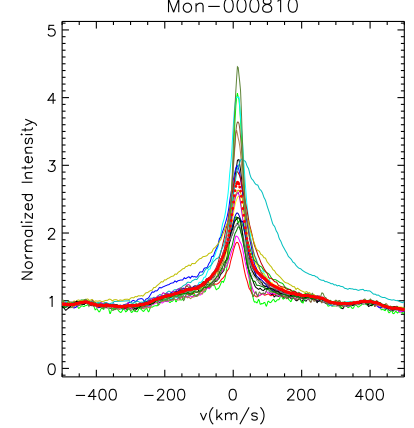

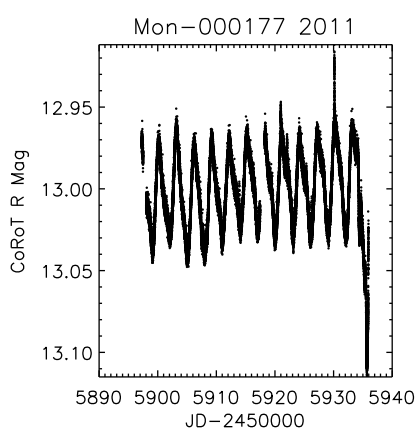

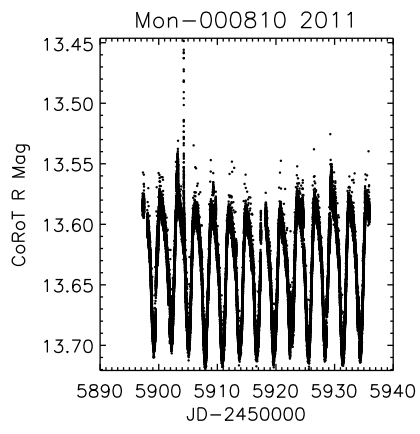

Fig. 16. Spectroscopic and photometric variability of spot-like systems. Left column: bidimensional periodogram of the H $\alpha$ line of two spot-like systems. The color code represents the power of periodogram, varying from zero (white) to the maximum power intensity (black). Black lines correspond to the photometric period of the stellar light curve observed with CoRoT in 2011. Middle column: H $\alpha$ line profiles. Different colors correspond to different nights of observation, and the thick red line is the average line profile. Right column: CoRoT light curve from 2011. None of the spot-like systems observed with FLAMES showed any periodicity in the $\mathrm{H} \alpha$ line.

not a globally organized accretion environment. This star also presents a similar periodicity ( $~ 8.1$ days) in the Spitzer (IRAC) light curve at $3.6 \mu \mathrm{m}$ and $4.5 \mu \mathrm{m}$ (Cody et al. 2014). Recently, Blinova et al. (2015) divided the unstable accretion regime into two subclasses: ordered unstable (one or two unstable tongues) and chaotic unstable (several unstable tongues). A star in the ordered unstable accretion regime may retain some periodicity in its accretion bursts, as may be the case for Mon-000996, which presents only a low-power period detection in its light-curve periodogram at about 8.7 days.

Mon-000558 was classified by Cody et al. (2014), using the same CoRoT data, as quasi-periodic, since its light curve includes low-amplitude stochastic variability superposed on some slightly periodic signal. The possible photometric period is $\sim 11.7$ days. In the literature, it also presents a period of 0.88 days (Flaccomio et al. 2006), which is very different from the $\mathrm{H} \alpha$ and the photometric period from our survey, however. We show in the next subsection that the $\mathrm{H} \alpha$ period of $\sim 10.5$ days is also seen in the HeI line (see Table 6), which may indicate that it corresponds to the stellar rotation period. This star had an accretion burst CoRoT light curve in 2008, while in 2011 it has an irregular, possibly hot-spot light curve. This is another system that is a candidate to be in the ordered unstable regime described by Blinova et al. (2015).

\subsection{Ho periodicity in stars not observed by CoRoT in 2011}

Two stars that show $\mathrm{H} \alpha$ line periodicity were not observed by CoRoT in 2011. The star Mon-001033, Fig. 15a, although not observed by CoRoT in 2011, shows a possible period of 14 days measured in 2011 with the Spitzer (IRAC) light curve in the CSI2264 campaign (Cody et al. 2014). This star was observed by CoRoT in 2008, and its light curve was classified as spot-like, with a period of 14.15 days, which agrees with the IRAC period, but is more than twice that obtained with the
$\mathrm{H} \alpha$ line ( $\sim 6.30$ days). The periodic region in $\mathrm{H} \alpha$ corresponds to a narrow velocity range, where a redshifted absorption is present in the spectrum.

The other star that presented a periodicity in $\mathrm{H} \alpha$, but was not observed by CoRoT, is Mon-000239. This star does not have any period information in the literature and is not periodic with Spitzer photometry. The detected period in $\mathrm{H} \alpha$ occurs in a very limited profile region associated with a small redshifted absorption feature, like the one observed in Mon-001033.

\subsection{Ho periodicity in spot-like stars}

Eight CTTSs classified as spot-like with the 2011 CoRoT light curves were observed with FLAMES. However, despite being periodic in the photometry, none of them showed any periodicity in the $\mathrm{H} \alpha$ line. Two examples are presented in Fig. 16. The photometric periodicity of spot-like stars is mostly due to cold spots on the photosphere, while the $\mathrm{H} \alpha$ line is mainly formed in the accretion funnel. Results from spectropolarimetry have shown that the main hot and cold spots tend to coincide in accreting stars (Donati et al. 2010, 2011a,b), and we would therefore expect the cold spot and the main accretion funnel, which ends in the hot spot, to present the same periodicity. At the same time, the $\mathrm{H} \alpha$ line profiles of spot-like systems tend to be weak and may therefore have a significant chromospheric contribution. In some spot-like systems, the lack of $\mathrm{H} \alpha$ line periodicity could then be due to a rather uniform chromospheric emission contributing significantly to the profile, which would dilute the $\mathrm{H} \alpha$ modulation from the accretion funnel.

\section{Periodograms of the Hel $6678 \AA$ A emission line}

The HeI $6678 \AA$ singlet line is often found in emission in stars with a moderate to high accretion rate (Muzerolle et al. 1998; Beristain et al. 2001; Luhman et al. 2003). Like the HeI $5876 \AA$ 


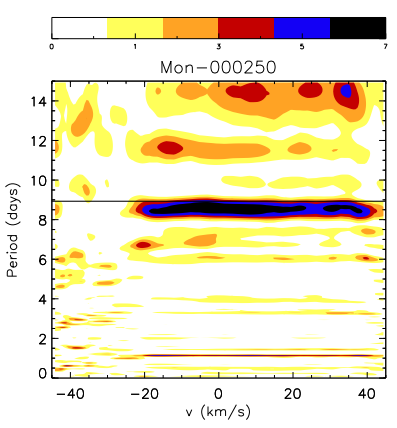

(a)

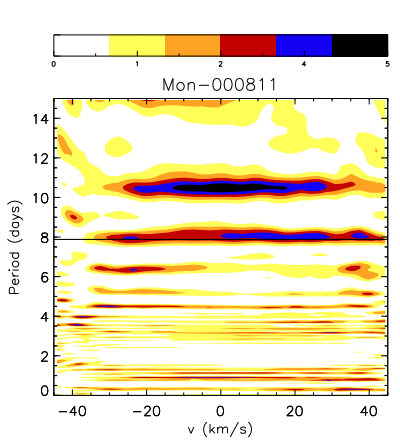

(e)

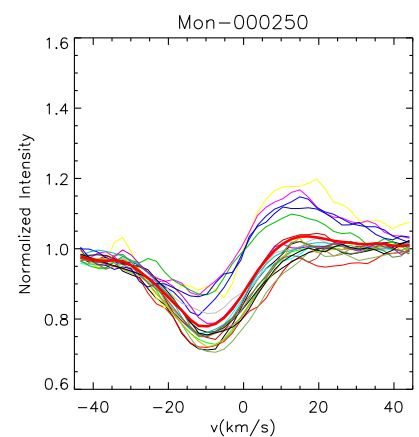

(b)

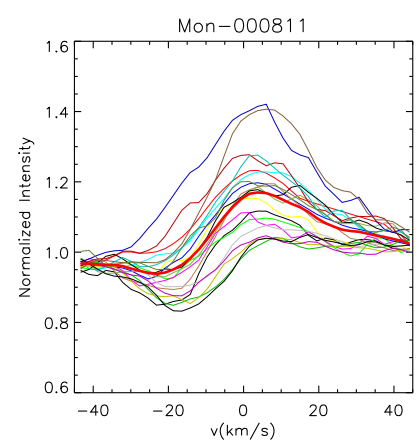

(f)

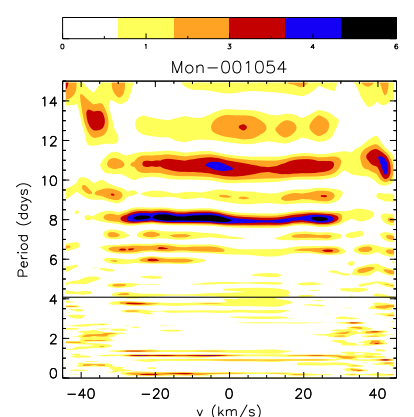

(c)

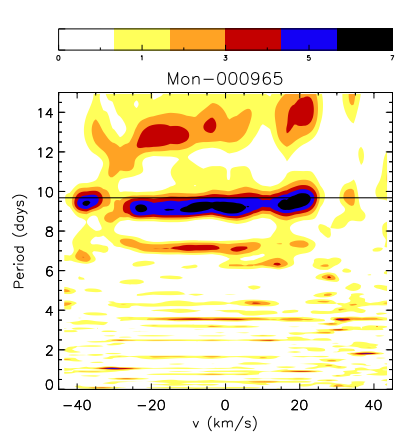

(g)

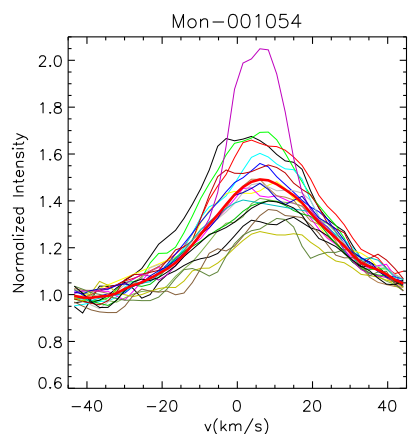

(d)

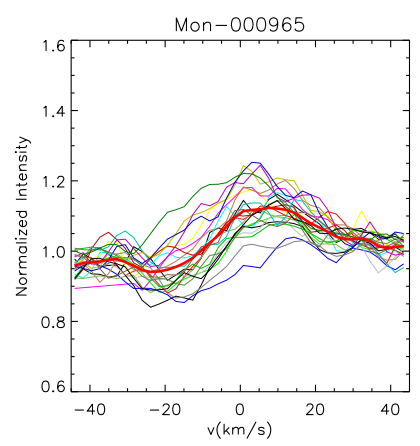

(h)

Fig. 17. Periodic stars in the HeI $6678 \AA$ that also showed a periodicity in the CoRoT light curves. Bidimensional periodograms of the HeI line (first and third columns). The color code represents the power of the periodogram, varying from zero (white) to the maximum power intensity (black). The black horizontal lines correspond to the period of the 2011 CoRoT light curve. The second and fourth columns present the HeI $6678 \AA$ lines used to calculate the periodograms. Different colors correspond to different nights of observation, and the thick red line is the average line profile.

triplet line, it may present a narrow (NC) and a broad (BC) component, with different kinematic characteristics. In the spectra of CTTSs, the NC is found to be slightly redshifted and circularly polarized and is interpreted to arise from gas in the post-shock region near the stellar surface (Beristain et al. 2001; Edwards et al. 2003). The BC is more complex. It can be found to be redshifted, but is most commonly observed blueshifted in CTTSs. The current interpretation is that it may come from the accretion funnel or the base of a hot accretion-powered stellar wind (Beristain et al. 2001; Edwards et al. 2006).

Depending on the star-disk inclination with respect to our line of sight, the hot spot may be hidden by the inner disk and remain invisible most of the time. In that case, we would not expect to see the HeI line in emission, even at high accretion rates. This is probably the case of the AA Tau-like system Mon000660 , which is seen at high inclination $\left(79^{\circ} \pm 11^{\circ}\right.$, Fonseca et al., in prep.) and does not present UV excess or HeI $6678 \AA$ in emission, despite being actively accreting, as shown by its extended $\mathrm{H} \alpha$ emission profile.

The HeI NC is formed close to the stellar surface, therefore its periodicity, whenever present, should correspond closely to the stellar rotation period. We expect to detect periodic variability if accretion is in a stable regime, which creates a main accretion funnel and consequently a main hot spot in each hemisphere.

We found that 24 of the 58 CTTSs observed with FLAMES clearly presented the HeI $6678 \AA$ line in emission. Another set of 11 CTTSs also show HeI in emission, but the signal-tonoise ratio is too low in the line to allow a reliable analysis of line profile variability. According to Beristain et al. (2001), the $\mathrm{HeI} \mathrm{NC}$ is characterized by line widths smaller than $50 \mathrm{~km} \mathrm{~s}^{-1}$. In our sample, the width of the mean HeI $6678 \AA$ line profile ranged from 29 to $47 \mathrm{~km} \mathrm{~s}^{-1}$, depending on the target, with a mean value of $39.3 \mathrm{~km} \mathrm{~s}^{-1}$. Therefore, in our sample, all of the stars that showed HeI $6678 \AA$ in emission only presented an NC. Close to the HeI $6678 \AA$ line, there is a photospheric absorption FeI line (at $6677 \AA$ ). We used residual spectra to calculate the HeI $6678 \AA$ line equivalent widths because of this line. The FeI $6677 \AA$ line was subtracted from these spectra, using as template the spectrum of a WTTS of the same spectral type of each star.

We calculated the HeI line periodograms as was done for the $\mathrm{H} \alpha$ line (see Sect. 8). The periodograms of the HeI lines are shown in Figs. 17 and 18. Of the 24 CTTSs with clear HeI emission, 12 stars showed no periodicity in this line, and they were also non-periodic in the CoRoT photometry. The non-periodicity of half of the HeI emission stars may indicate the intermittent formation of several short-lived hot spots near the stellar surface, instead of one well-organized hot spot in each hemisphere.

Of the 12 systems that showed a periodicity in the HeI line, two presented more than one period in this line (as seen in Table 6 and Figs. 18e and i), which may be because of the formation of several long-lived (compared to the time span of our observations) hot spots at different latitudes on the stellar surface, rather than a main hot spot on each hemisphere. In the next subsections, we present the analysis of the HeI line periodicity compared to the CoRoT light-curve morphology discussed in Sect. 4. 


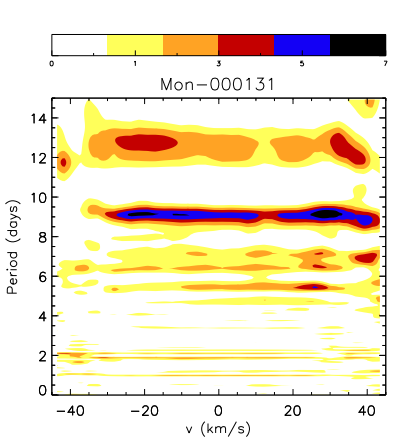

(a)

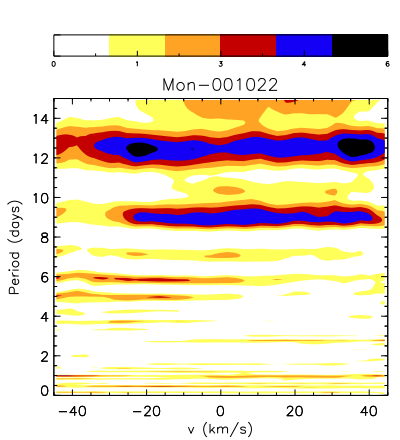

(e)

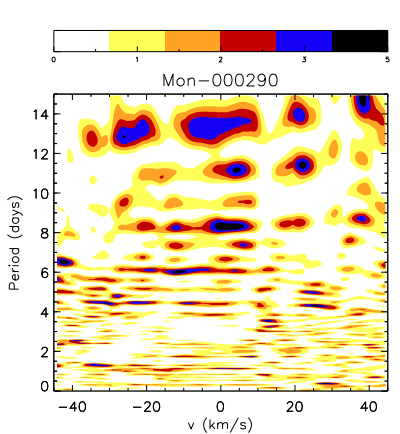

(i)

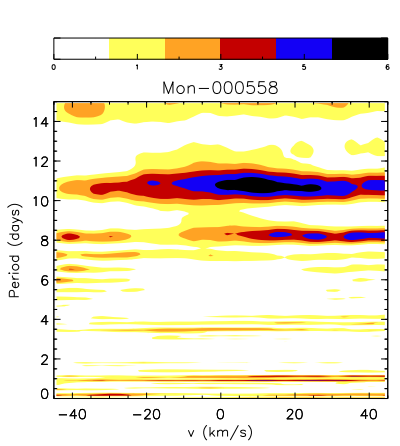

(m)

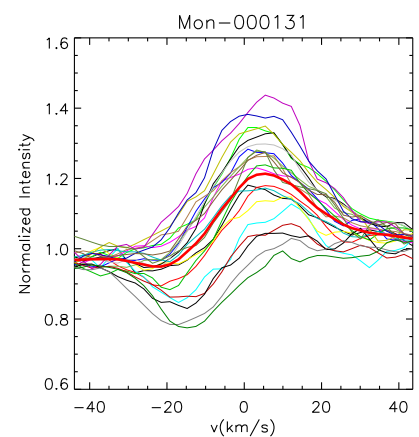

(b)

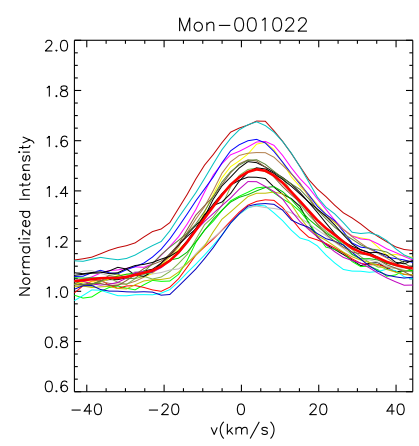

(f)

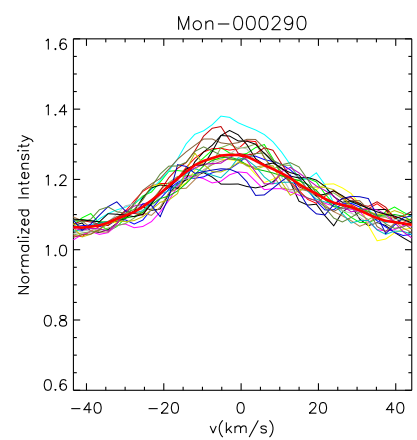

(j)

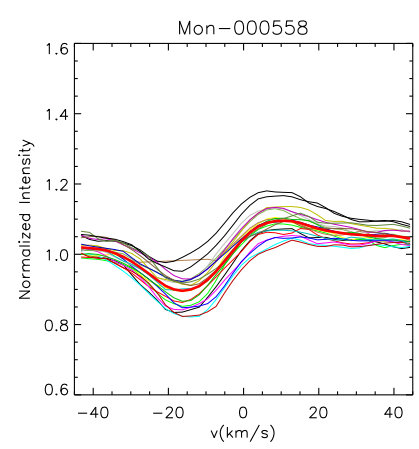

(n)

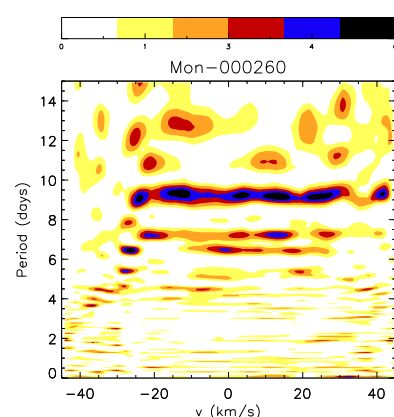

(c)

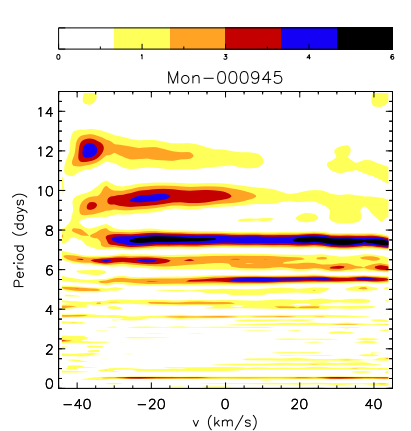

(g)

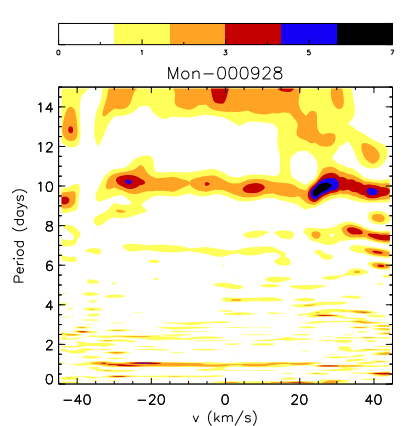

(k)

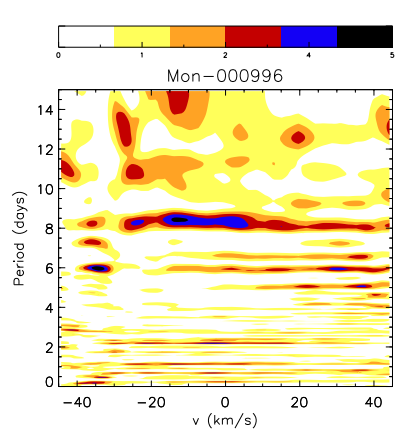

(o)

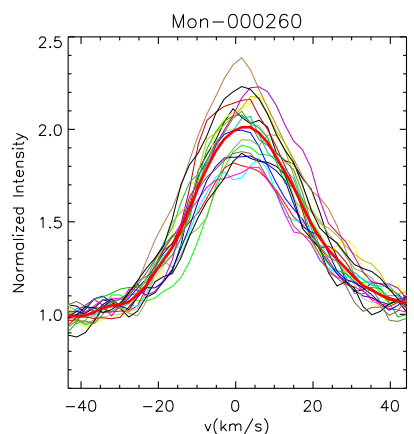

(d)

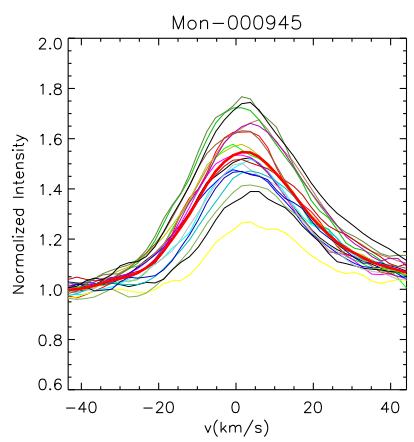

(h)

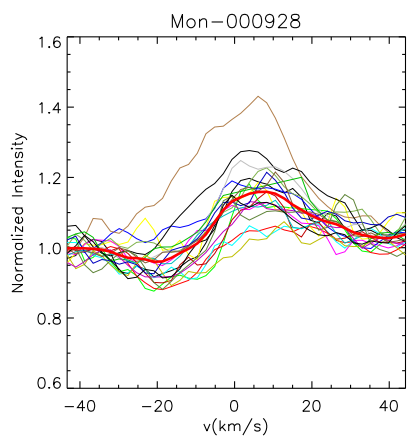

(1)

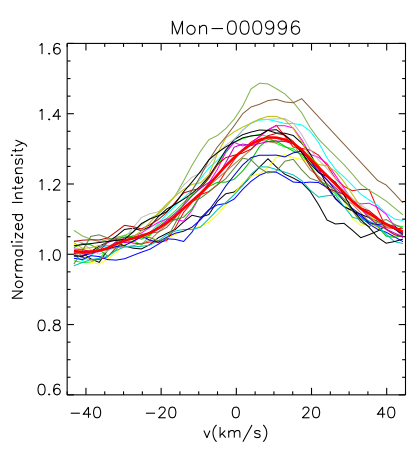

(p)

Fig. 18. Periodic stars in the HeI $6678 \AA$ line that did not show a periodicity in the CoRoT light curves. The first and third columns show bidimensional periodograms of the HeI line. The color code represents the power of the periodogram, varying from zero (white) to the maximum power intensity (black). The second and fourth columns show the HeI $6678 \AA$ Aines used to calculate the periodograms. Different colors correspond to different nights of observation, and the thick red line is the average line profile.

\subsection{Hel line in AA Tau like stars}

AA Tau-like stars are seen at high inclination with respect to our line of sight, and the inner disk is expected to hide part of the stellar photosphere as the system rotates. Depending on the system geometry, the hot spot, and consequently the region where the HeI NC forms, may also be hidden in these systems. Only three of eight AA Tau-like stars observed with FLAMES showed 
the HeI line in emission, and these three systems presented a periodicity in this line. The HeI line periodograms of Mon-000250, Mon-001054, and Mon-000811 are shown in Fig. 17 and the measured periods are presented in Table 6.

Mon-000250 shows the same periodicity in $\mathrm{HeI}$ and $\mathrm{H} \alpha$ lines (Figs. 17a and 13d), and also presents the same period in the CoRoT light curve, which confirms that the structure responsible for the stellar occultation is located close to the co-rotation radius of the star-disk system.

Mon-001054 (Fig. 17c) shows a HeI period of $\sim 8.1$ days, that is, twice the CoRoT photometric period from 2011. The photometric period, calculated with the modified Scargle periodogram, as explained in Sect. 4, presents a low detection power. Using a different period search method, such as the autocorrelation function (Cody et al. 2014; McQuillan et al. 2013), we find a $\sim 8.2$ day period in the CoRoT light curve, but the detection significance is again very low. From the literature, Mon-001054 has a photometric period of $\sim 7.8$ days (Lamm et al. 2004), which agrees with our HeI period and could correspond to the stellar rotation period.

Mon-000811 (Fig. 17e) is periodic in $\mathrm{HeI}(\sim 10.5$, higher power and $\sim 8$ days lower power) and $\mathrm{H} \alpha$ ( $\sim 12.5$ days $)$ (Figs. 17e, 13g) and shows periodicity in the CoRoT light curve ( 7.88 days). However, these periods are different from each other. As discussed above, this star is classified as AA Tau-like, and we would therefore expect accretion to occur in a stable regime, if the inner disk warp is associated with the base of the accretion column, and forming a major hot spot in each hemisphere at the top of the accretion column. One possible scenario to explain this complex system is that the HeI line eight-day period corresponds to the stellar rotation period, which is close to the photometric period, and therefore the inner disk warp that causes the photometric variability is located close to the corotation radius. The $\mathrm{H} \alpha \sim 12.5$-day period is observed in the blue wing of the emission profiles and could then correspond to emission or absorption from a wind located outside the corotation radius.

\subsection{Hel line periodicity in spot-like stars}

Because of the high stability of the spot-like light curves on the timescale of our observations and the low mass-accretion rates of these systems, we initially assumed that large cold spots were the main cause of the observed photometric variability of spotlike systems. If, instead, hot spots were causing the photometric variability, we might expect to observe the same type of variability in the HeI line. We observed eight spot-like systems with FLAMES, and only one, Mon-000965, has the HeI line in emission, as seen in Table 6. The HeI periodogram of this star is shown in Fig. $17 \mathrm{~g}$, and it presents a clear periodicity that coincides with the period obtained with the CoRoT photometry. Mon-000965 is not clearly periodic in $\mathrm{H} \alpha$ (Sect. 8.4), but a faint periodic signal (low power in the periodogram) is seen at the photometric period. The fact that this star has the HeI NC line in emission indicates that the hot spot is prominent, which suggests that its light-curve variability might be dominated by a stable hot spot instead of a cold spot, as initially assumed during our 2011 campaign.

\subsection{Periodic stars in the Hel line that are not periodic in the CoRoT photometry or were not observed by CoRoT in 2011}

Half of the 24 stars that showed HeI in emission presented periodicity in this line, but only 4 (Sects. 9.1 and 9.2) were also periodic in the CoRoT photometry, as seen in Fig. 17. The other 8 stars that showed a periodicity in the HeI line were not found to be periodic in the CoRoT light curves of 2011 (6 stars) or were not observed with CoRoT in 2011 (2 stars), as seen in Fig. 18.

All of the accretion burst systems observed by FLAMES (five in total) are included in the stars that showed HeI in emission, which is reasonable, since they all presented high mass-accretion rates and had light curves dominated by hot spot variability (Stauffer et al. 2014). Three of these five stars (Mon-000996, Mon-001022 and Mon-000945) presented a periodicity in the HeI line, despite showing no periodic signal in the CoRoT photometry. Mon-000996 (180) also presented similar periodicities in both the $\mathrm{HeI}$ and $\mathrm{H} \alpha$ lines, as shown in $14 \mathrm{~d}$.

\section{Stable and unstable accretion}

CTTSs are photometrically variable on different timescales, from seconds to even decades (Rucinski et al. 2008), depending on the various physical phenomena that affect the stellar and circumstellar environment. Variable accretion is one of the main sources of variability in this evolutionary phase. As discussed by Kurosawa \& Romanova (2013), accretion can occur in stable and unstable regimes. The unstable regime may be triggered by the Rayleigh-Taylor instability, which takes place in the inner disk region. This type of variability produces non-periodic light curves that are dominated by accretion bursts, as observed in some stars in NGC 2264 (see Stauffer et al. 2014). This variability also produces detectable spectral features in emission lines, such as $\mathrm{H} \beta, \mathrm{H} \gamma$, and $\mathrm{Pa} \beta$ (Kurosawa \& Romanova 2013).

According to theoretical predictions, stars in a stable accretion regime will present redshifted absorption in just a few rotational phases, when the main hot spot is seen projected through the accretion funnel in our line of sight. In this situation, photons emitted by the hot spot will be absorbed by the high-velocity gas in the accretion funnel, causing the observed redshifted absorption components. In the unstable accretion regime, redshifted absorption may be seen at any rotational phase because of the large number of accretion funnels, and consequently, hot spots on the stellar surface. The accretion burst stars are all expected to be in the unstable accretion regime. Unfortunately, only five accretion burst systems were included in our sample of stars observed with FLAMES, and none of them showed redshifted absorption in $\mathrm{H} \alpha$. Their $\mathrm{H} \alpha$ line profiles are dominated by emission due to accretion (Reipurth type I profile) that typically does not present redshifted absorption (see Fig. 12). To investigate the occurrence of redshifted absorption in the emission lines of accretion burst systems, we need to analyze higher order Balmer lines, as suggested by Kurosawa \& Romanova (2013), or the HeI (10 $830 \AA$ ) line, which often shows both redshifted and blueshifted absorption in CTTS spectra, as discussed by Edwards et al. (2006) and Cauley \& Johns-Krull (2014).

In the simulations by Kurosawa \& Romanova (2013), the redshifted absorption components are not visible in the $\mathrm{H} \alpha$ line either because its emission is very intense and hides the shallow absorption. Redshifted absorption is most commonly seen observationally in weaker Balmer emission lines such as $\mathrm{H} \beta$ and $\mathrm{H} \gamma$. We do not have the higher Balmer lines in our spectra, but ten stars show a clear redshifted absorption component in the $\mathrm{H} \alpha$ line (marked in Col. 8 of Table 6). Despite the small number of targets, we tried to verify how the redshifted absorption component varies with stellar rotational phase and compare our results with the prediction of stable and unstable accretion simulations. 


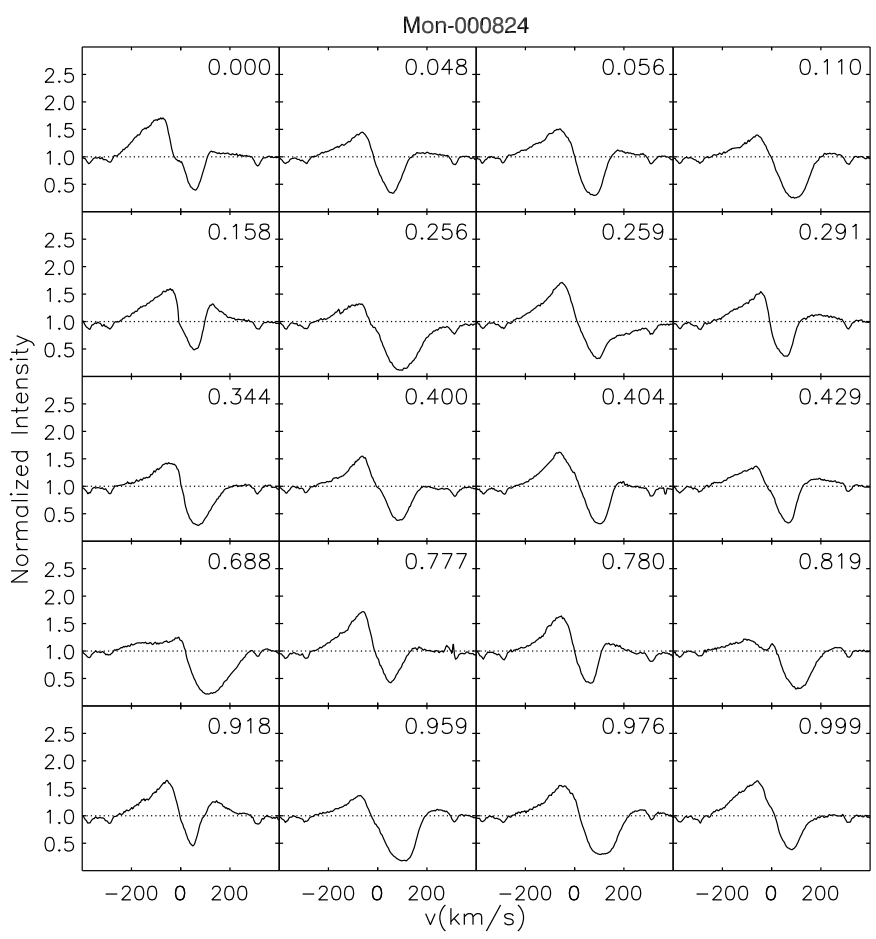

Fig. 19. $\mathrm{H} \alpha$ line of Mon-000824 as a function of rotational phase, indicated in each plot. This star was not observed by CoRoT in 2011, and we used the photometric period (7.05 days) obtained in 2008, when the star presented an AA Tau-like light curve, to calculate the rotational phases. This star is a candidate to be in the unstable accretion regime in 2011.

Of our ten selected targets, only Mon-000824 is possibly in the unstable accretion regime, where the redshifted absorption is present in the line profile at all rotational phases. This star was classified as AA Tau-like in 2008, but was not observed by CoRoT in 2011 (see Fig. 19), which means that in principle we would not know its photometric behavior when the spectroscopic data were obtained. As seen in Sect. 4, CTTSs are highly variable and in a few years may change the morphology of the their light curve from AA Tau to non-periodic and vice versa. Although Mon-000824 was not observed with CoRoT in 2011, during the CSI campaign about 900 epochs of I-band photometry were obtained from November 2011 to March 2012 of the central region of NGC 2264, including Mon-000824, with the US Naval 40" telescope. As discussed by McGinnis et al. (2015), the simultaneous I-band and CoRoT photometry typically match very well. We therefore inspected the $I$-band light curve of Mon-000824 in 2011 and found that it was indeed not periodic, implying that this star probably changed from a stable to an unstable accretion regime from 2008 to 2011.

The other nine selected stars (two spot-like, five AA Taulike, one aperiodic extinction variables and one not observed by CoRoT in 2011) are apparently in a stable accretion regime, showing redshifted absorptions in only a few rotational phases. In Fig. 20, we show the spectra of Mon-000296 as an example.

The predictions that stable and unstable accretion regimes present a different rotational phase distribution of redshifted absorption components are based on the theoretical line profiles computed by Kurosawa \& Romanova (2013) for a system with an inclination of $60^{\circ}$ with respect to the line of sight. However, in systems with lower inclinations, hot spots will always be in view (Kurosawa et al. 2008), hence, even in a stable accretion regime the redshifted absorption may be present in the line profile at all

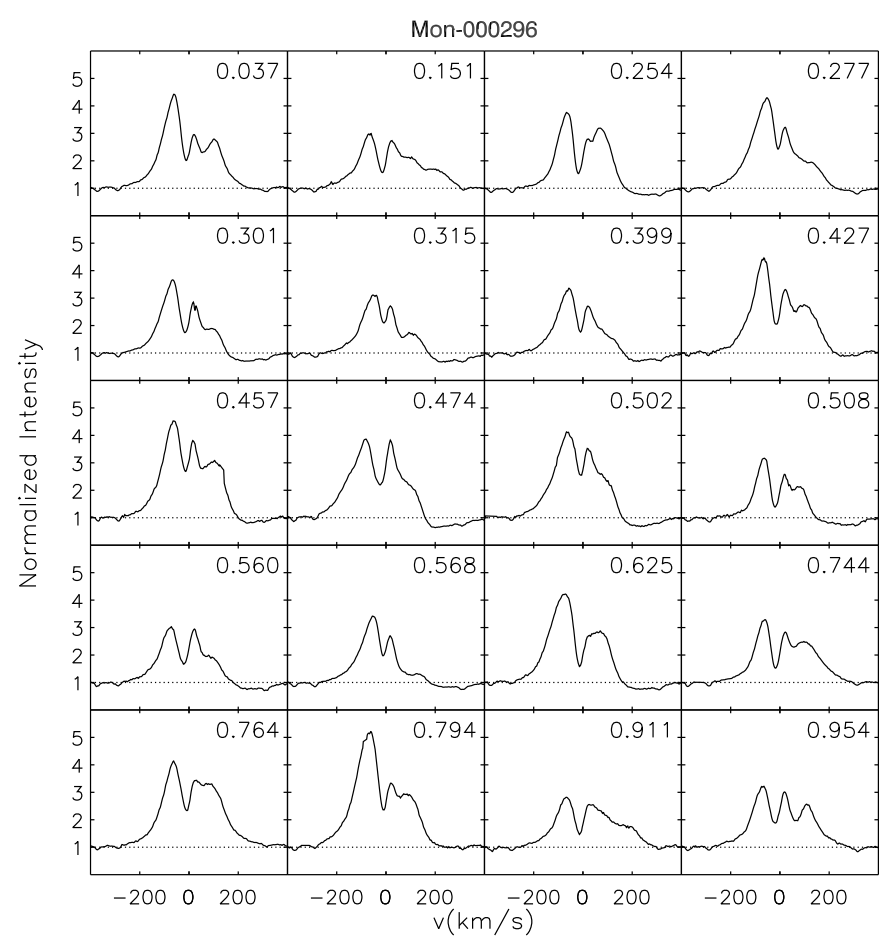

Fig. 20. $\mathrm{H} \alpha$ line of Mon-000296 as a function of rotational phase, indicated in each plot. The central peak is the nebular line that was not entirely removed. This star presents an AA Tau-like light curve with a 3.91 day period and is a candidate to be in the stable accretion regime in 2011.

rotational phases. This is, however, not the case of Mon-000824 (Fig. 19), since its inclination with respect to our line of sight is $72^{\circ} \pm 5^{\circ}$ (McGinnis et al. 2015).

We would expect stars in a stable accretion regime to show periodic light curves and stars in an unstable accretion regime to show non-periodic light curves (Kurosawa \& Romanova 2013; Kulkarni \& Romanova 2008, 2009). This is because the stable accretion regime generates an organized circumstellar environment that shows periodicity as the star-disk system rotates. Of the nine stars we classified as being in a stable regime, using this criterion of redshifted absorption, only two did not present periodic light curves, as seen in Table 6. Instead their light curves were classified as aperiodic extinction variables. The stable accretion regime classification therefore agrees with the observation of periodicity in the CoRoT light curves for most stars with redshifted absorption in just a few rotational phases.

In the stable accretion regime, the variability of the redshifted absorption is expected to be periodic. Of the nine stars we classified as being in a stable regime, five are periodic in the $\mathrm{H} \alpha$ line, agreeing with the theoretical predictions of Kurosawa \& Romanova (2013). This means that only about half of the stars we classified as being in a stable accretion regime are periodic in the $\mathrm{H} \alpha$ line. The only star in the unstable accretion regime (Fig. 19) is also not periodic in the $\mathrm{H} \alpha$ line, as predicted by Kurosawa \& Romanova (2013).

The classification of a stable accretion regime based on the presence of redshifted absorption at well-defined rotational phases agrees only sometimes with the type of observed variability in the CoRoT light curves. Neither is a star that shows redshifted absorption at all rotational phases necessarily accreting in the unstable regime. Other factors, such as the inclination of the system, may influence the presence of the redshifted absorption in the Balmer lines. Moreover, we must keep in mind that 
the $\mathrm{H} \alpha$ line is not the best choice for this type of analysis, since other lines, such as $\mathrm{H} \beta, \mathrm{H} \gamma$, and $\mathrm{Pa} \beta$, are weaker, and the redshifted absorption is more commonly visible in the line profiles.

\section{Correlation matrices}

The $\mathrm{H} \alpha$ emission line can be formed in several different regions, in the accretion funnel, the chromosphere, and/or the wind. The analysis of the correlation of different parts of the $\mathrm{H} \alpha$ line profile can help us investigate if the line comes from different regions and if these regions are correlated.

One method of checking the correlation across a line profile is to calculate correlation matrices. We divided each $\mathrm{H} \alpha$ profile into small velocity intervals of $1.5 \mathrm{~km} \mathrm{~s}^{-1}$ and calculated the linear correlation coefficient, $r(i, j)$, of the line intensity at each velocity interval $i$ with each velocity interval $j$, as described by Johns \& Basri (1995a). The correlation coefficient ranges from -1 to 1,1 corresponding to a perfect correlation, 0 to no correlation, and -1 to a perfect anticorrelation. When $i=j$, $r(i, j)=1$, and for all values of $i$ and $j, r(i, j)=r(j, i)$, implying that the matrix is symmetrical relative to the diagonal. We present the result as a color-map plot of the correlation coefficients, which is useful to visually identify regions of the profile that are correlated or anticorrelated. This has been used in the literature to analyze correlations across the $\mathrm{H} \alpha$ line and other lines such as H $\beta$ (Johns \& Basri 1995b; Oliveira et al. 2000; Alencar \& Batalha 2002; Kurosawa et al. 2005).

All of the 58 CTTSs that have FLAMES spectra present some correlation signal of the red wing with itself. Some stars show this correlation just in the redshifted absorption position and others just in the redshifted emission. We find that $\sim 74 \%$ of the CTTSs show a very good correlation across the entire $\mathrm{H} \alpha$ red wing, which then varies coherently. Almost all the CTTSs observed by FLAMES also exhibited some correlation of the blue wing with itself, $\sim 74 \%$ presenting a substantial correlation. The blue wing of $\mathrm{H} \alpha$ also varies coherently. This shows that the variability in each wing of the $\mathrm{H} \alpha$ profile is coherent, which indicates that each line wing (red or blue) is formed in a region dominated by a given physical process.

We find that $\sim 34 \%$ of the CTTSs observed with FLAMES show no sign of correlation between the red and the blue wings, implying that they vary independently in those systems. At the same time, $\sim 66 \%$ CTTSs show some correlation of the red wing with the blue wing and among these, $\sim 55 \%$ show substantial correlation across the entire wings, while $\sim 45 \%$ show correlations that occur only in some specific velocity range.

Only 7 of the 58 CTTSs observed with FLAMES present anticorrelated variations in the $\mathrm{H} \alpha$ line profile. The anticorrelation seen in 4 of these 6 stars is associated with a blue- or redshifted absorption that varies in antiphase with the rest of the profile. The other three stars present an anticorrelation associated with a blueshifted emission and the other profile parts.

Overall, the $\mathrm{H} \alpha$ line profiles present variations that cannot be explained by continuum variability alone that is due, for example, to hot spots. If excess continuum emission from hot spots were the main cause of emission line variability, the entire profile would be correlated, and the $\mathrm{H} \alpha$ line would not vary in shape, but only in intensity. Other factors such as wind emission or absorption, accretion variability, and non-symmetrically distributed circumstellar material must influence the emission line variability, often simultaneously, since many stars do not present a very good correlation across the entire $\mathrm{H} \alpha$ line profile. It is possible, however, that in some cases the $\mathrm{H} \alpha$ line may be formed mainly in one specific region, like the wind or the accretion column. We

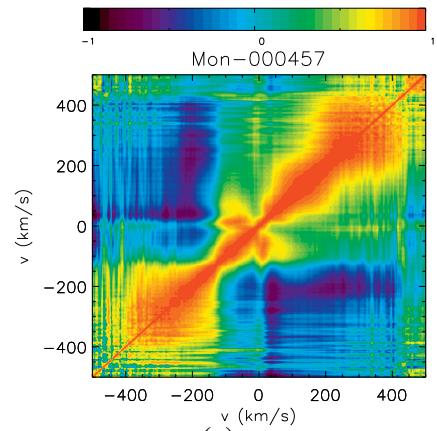

(a)

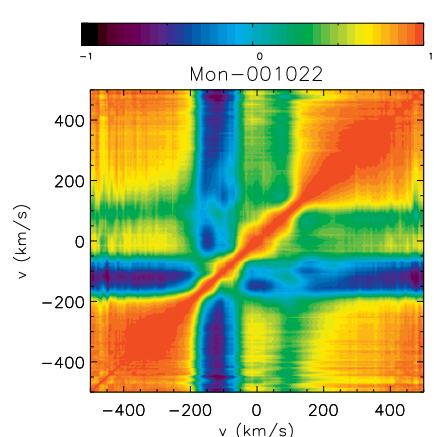

(c)

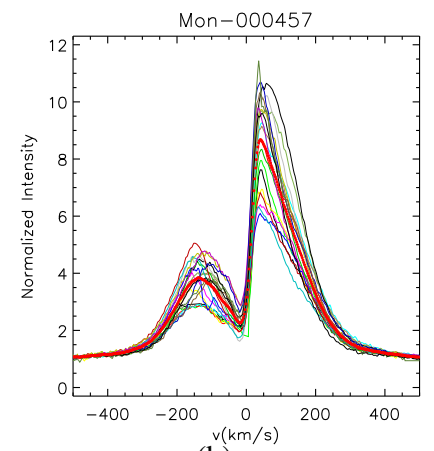

(b)

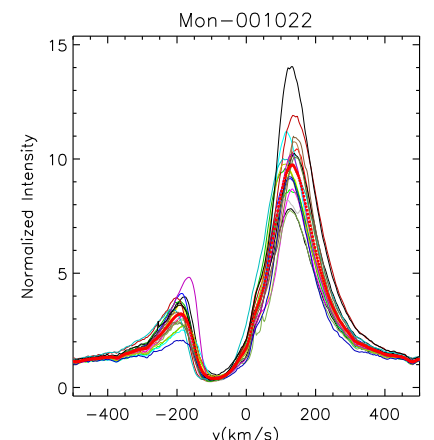

(d)
Fig. 21. Examples of correlation matrices (left) of the $\mathrm{H} \alpha$ line of two CTTSs and the corresponding line profiles (right). In panels a) and c), the color range corresponds to the value of the linear correlation coefficient between the different velocity bins of the $\mathrm{H} \alpha$ line profiles. Perfect anticorrelation corresponds to -1 (black), no correlation to 0 (light blue), and a perfect correlation to 1 (orange). In panels b) and d), different colors correspond to spectra observed in different nights, and the thick red line is the average line profile. The correlation matrices and $\mathrm{H} \alpha$ line profiles of all the CTTSs in our sample observed with FLAMES are presented in the Appendix.

would then expect the line profile to be dominated by the same physical process and the variability of the entire line profile to be correlated.

Figures 21 and 22 show examples of correlation matrices for some CTTSs, together with their observed $\mathrm{H} \alpha$ line profiles. These figures are representative of the different types of correlation matrices we obtained. In these plots, the correlation coefficients are represented by colors ranging from black $(r=-1$, maximum anticorrelation) to light blue $(r=0$, no correlation) and to orange ( $r=1$, maximum correlation). The correlation matrices and $\mathrm{H} \alpha$ line profiles of all the CTTSs in our sample observed with FLAMES are presented in the Appendix.

Figure 21a,b shows that for Mon-000457 the red wing of $\mathrm{H} \alpha$ correlates well with itself in the velocity range of $50 \mathrm{~km} \mathrm{~s}^{-1}<v<350 \mathrm{~km} \mathrm{~s}^{-1}$, and the blue wing shows a good correlation with itself at $-350 \mathrm{~km} \mathrm{~s}^{-1}<v<-75 \mathrm{~km} \mathrm{~s}^{-1}$. We can also see a region of correlated variability in the blue wing at $-100 \mathrm{~km} \mathrm{~s}^{-1}<v<0 \mathrm{~km} \mathrm{~s}^{-1}$, which corresponds to the blueshifted absorption from a wind that varies quite independently of the rest of the profile. The blue wing is anticorrelated with the red wing for velocities in the range of $\sim-100$ to $\sim-350 \mathrm{~km} \mathrm{~s}^{-1}$ and $\sim 0$ to $\sim 350 \mathrm{~km} \mathrm{~s}^{-1}$. The line profiles show that these regions correspond to the blue and red emissions that vary in antiphase with each other. This indicates a strong influence of different physical processes in each $\mathrm{H} \alpha$ profile wing, the red wing is probably dominated by accretion and the blue wing by the wind. The accretion process and the wind may contribute to the entire profile, but can have a stronger contribution 


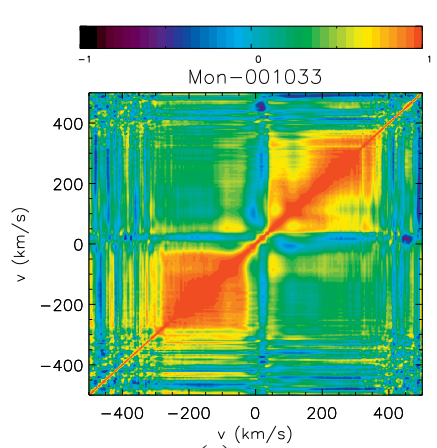

(a)

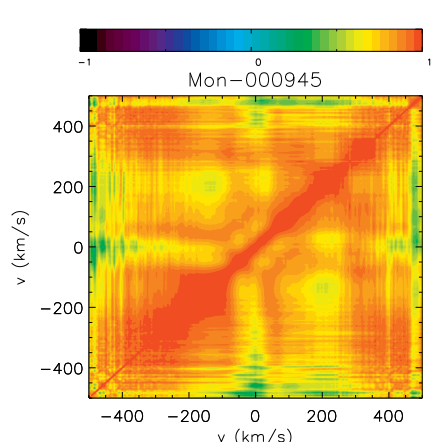

(c)

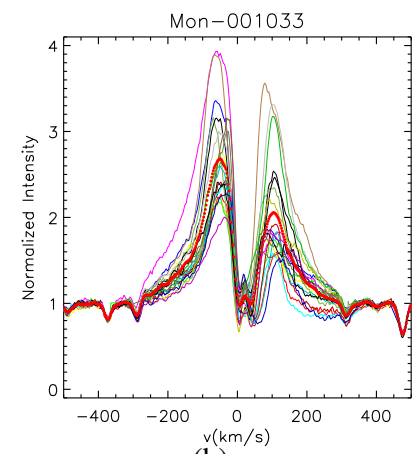

(b)

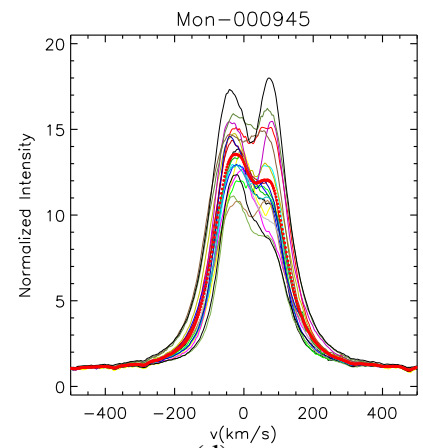

(d)
Fig. 22. Examples of correlation matrices (left) of the $\mathrm{H} \alpha$ line of two CTTSs and the corresponding line profiles (right). The color code is the same as in Fig. 21.

in one particular velocity range, as seen for example in theoretical line profiles calculated by Kurosawa et al. (2006) and Lima et al. (2010), which include both the wind and accretion contributions. In the case of Mon-000457, if the blue wing is mostly influenced by the wind and accretion is probably the main process acting on the red wing, the anticorrelation between the two wings could be explained as being due to rotational modulation of a CTTS with an inclined magnetosphere, as also observed in SU Aur (Johns \& Basri 1994). When the main accretion funnel faces the observer, accretion is strong and ejection is low, and half a rotation cycle later, the opposite occurs. In an ideal case, these variations should be periodic, but the $\mathrm{H} \alpha$ line variability of this star does not show very clear signs of periodicity.

Figure 21c shows a strong correlation of the red wing with itself and with the blue wing of the $\mathrm{H} \alpha$ profile at high velocities $\left(|v|>200 \mathrm{~km} \mathrm{~s}^{-1}\right)$ for Mon-001022. At the same time, the strong blueshifted absorption $\left(-200 \mathrm{~km} \mathrm{~s}^{-1}<v<0 \mathrm{~km} \mathrm{~s}^{-1}\right)$ is anticorrelated with the blue and red emission regions of $\mathrm{H} \alpha$. This feature is due to photons from the accretion columns and a hot spot absorbed by the outflowing disk wind material in our line of sight. Stars that present very strong blueshifted absorption in $\mathrm{H} \alpha$ normally present emission profiles dominated by the wind, as can be seen in the theoretical profiles calculated by Kurosawa et al. (2006) and Lima et al. (2010). When the emission due to the wind increases, photons are even more strongly absorbed by the wind, increasing the depth of the blueshifted absorption. This causes the blueshifted absorption to become anticorrelated with the rest of the emission line.

Figure 22a shows the correlation matrix and the $\mathrm{H} \alpha$ line profiles of Mon-001033. The blue and red wings of the $\mathrm{H} \alpha$ profile correlate well with themselves, but show no correlation or anticorrelation with each other. The emission component is quite variable, not very intense, and suffers strong influence from absorption in the blue and red wings. Blueshifted absorption is associated with winds and redshifted absorption with the accretion funnel. The combination of faint emission and strong absorption that probably comes from different physical processes created a variability pattern where the variations of one wing are not correlated with the other wing.

Theoretical correlation matrices are rare in the literature. In Alencar et al. (2012), H $\alpha$ correlation matrices were calculated from theoretical line profiles that included only the magnetospheric contribution. Their theoretical correlation matrices showed a good correlation of the entire $\mathrm{H} \alpha$ line profile, but outflows were not taken into account. Our observed CTTSs include stars such as Mon-000945, whose $\mathrm{H} \alpha$ emission line profiles show no hint of blueshifted absorption and should be dominated by accretion. Figures 22c, d show the $\mathrm{H} \alpha$ profiles of Mon-000945 and its corresponding correlation matrix, which indeed is well correlated across the entire profile, as predicted theoretically by magnetospheric accretion models. However, the $\mathrm{H} \alpha$ profile of most CTTSs cannot be explained by magnetospheric accretion alone, and it would be very interesting to be able to compare the observed correlation matrices with theoretical matrices calculated from profiles that include both accretion and outflows.

Veiling variations would also produce a correlation across the entire line profile, as observed in the H $\alpha$ line of Mon-000945. However, the $\mathrm{H} \alpha$ line profile presents changes in its morphology, not only in its intensity (see Fig. 22d), as would be expected for variations that are only due to the extra veiling continuum. The type of correlated variability and $\mathrm{H} \alpha$ profile morphology observed in Mon-000945 is also seen in the CTTSs T Tau (Johns \& Basri 1995b) and TW Hya (Alencar \& Batalha 2002), for which it has also been suggested that the $\mathrm{H} \alpha$ line is mostly formed in the accretion columns.

The analysis of the correlation matrices shows that the $\mathrm{H} \alpha$ line variability can be associated with different physical processes. The variability of the red wing often does not affect the variability of the blue wing. This is confirmed by the lack of correlation of the blue wing with the red wing, seen in $\sim 34 \%$ of the $\mathrm{H} \alpha$ correlation matrices. This lack of correlation disagrees with theoretical models that only include the magnetospheric accretion contribution to the emission line profile. It would be very interesting to compare observational correlation matrices to matrices calculated from hybrid magnetosphere and wind models, such as those of Kurosawa et al. (2006, 2011), and determine which circumstellar environment would be able to reproduce the observational matrices. At the same time, the red wing is almost always well correlated with itself, as is the blue wing with itself, indicating that the variability of each wing is dominated by the same physical phenomenon.

\section{Interesting objects}

Some objects are interesting enough to be analyzed individually in future work. We found three spectroscopic binaries with the FLAMES data. The CTTS Mon-000448 is a double-line spectroscopic binary, while the WTTS Mon-000497 and the CTTS Mon-000804 are single-line spectroscopic binaries. These stars do not present eclipses in the CoRoT light curves, however.

The AA Tau-like star Mon-00811 is a CTTS that has different periods in different accretion and circumstellar features. One possible interpretation is that the different periods come from different regions of the star-disk system. It has a photometric period ( $\sim 7.9$ days) due to occultation of the star by the inner disk, 
a stellar rotation period ( $\sim 8$ days) obtained from the HeI line periodogram, a 10.5-day period from the HeI blueshifted emission, and a period of $\sim 13$ days in the $\mathrm{H} \alpha$ line blue wing that we associate with a disk wind.

Some WTTSs have strong IR excesses, but do not show a clear sign of accretion (Mon-001157, Mon-000434, Mon000279, Mon-000753, and Mon-000271). For some unknown reason, accretion has been shut off or is at a very low level, despite the available material in their inner disks. It would be interesting to reobserve these systems to verify whether accretion features reappear in the future, of if it has indeed come to an end.

\section{Conclusions}

We investigated accretion in a group of classical $\mathrm{T}$ Tauri stars that belong to the young cluster NGC 2264, using observational data obtained in the Coordinated Synoptic Investigation of NGC 2264 campaign. The main results of this work are listed below.

1. The light curves observed by CoRoT were morphologically classified as spot-like, AA-Tau-like, or non-periodic. Of the non-periodic light curves, some are due to accretion bursts, others to circumstellar disk obscuration, but most non-periodic light curves are very complex and could not be associated with a major physical phenomenon. In the 2011 campaign, we found $\sim 13 \%$ spot-like, $\sim 12 \%$ AA Taulike, and $\sim 70 \%$ non-periodic systems. Of the non-periodic ones, $\sim 19 \%$ were associated with accretion bursts, $\sim 13 \%$ with occultation by circumstellar dust, and $\sim 68 \%$ presented variability of unknown origin.

2. Of the CTTSs observed by CoRoT, $\sim 43 \%$ and $\sim 30 \%$ showed periodic photometric variability in 2008 and 2011, respectively. The number of periodic stars did not change significantly between the two runs, but a larger number of nonperiodic stars were included in the 2011 campaign.

3. Of the 84 stars that were observed with the CoRoT in the two different epochs (2008 and 2011), 30\% showed substantial variability in their light-curve morphology, changing to a different category in our light-curve classification scheme. These changes show how dynamic the CTTS circumstellar environment is in only a few years.

4. When UV excesses are not available, mass-accretion rates can be obtained with good results from the $\mathrm{H} \alpha$ flux using the currently available calibrations from the literature.

5. Most of the CTTSs we observed showed no periodicity in the $\mathrm{H} \alpha$ line. Only eight stars in our sample of 58 CTTSs observed with FLAMES were periodic in $\mathrm{H} \alpha$.

6. The HeI $6678 \AA$ narrow component line variability can provide the rotation period of a CTTS when the star is in a stable accretion regime, since in that case a major hot spot is expected to form in each stellar hemisphere. We found that 24 of the 58 stars observed by FLAMES had this line in emission. Of these, 12 were periodic in the HeI line, and 4 were also periodic photometrically.

7. None of the photometrically periodic spot-like systems that were observed spectroscopically presented a periodicity in $\mathrm{H} \alpha$, while spectropolarimetry results show that hot and cold spots tend to be coincident in CTTSs, and we might expect to observe the same periodicity in the cold spot and accretion funnel diagnostics. Spot-like systems tend to present low mass-accretion rates, and the lack of periodicity in the $\mathrm{H} \alpha$ line variability of spot-like systems could be due to chromospheric emission contributing significantly to the emission profile in these systems, which would dilute the $\mathrm{H} \alpha$ modulation from the spot.

8. We analyzed which CTTSs were in stable or unstable accretion regimes. Kurosawa \& Romanova (2013) suggested that CTTSs in a stable accretion regime should present redshifted absorption components in emission line profiles only in a few rotational phases, when photons from the hot spot are seen in our line of sight projected against the main accretion column. Systems in unstable accretion will produce random accretion tongues and should present redshifted accretion components at any rotational phase. We analyzed the ten CTTSs that showed redshifted absorption components in $\mathrm{H} \alpha$ and for which we had some information about the rotational period. Of these, nine CTTSs fulfilled the stable accretion criteria (five stars were AA Tau-like, two stars were spotlike, one was non-periodic, and one was not observed in 2011 by CoRoT), and in almost all the systems accretion in a stable regime agrees with the type of variability observed in the CoRoT light curves. Only one star of the ten selected CTTSs was apparently in the unstable accretion regime, presenting redshifted absorption at all rotational phases and showing no periodicity in $\mathrm{H} \alpha$, as expected.

9. The analysis of correlation matrices showed that the variability in the $\mathrm{H} \alpha$ line can be associated with different physical processes, and the red and blue wings are sometimes formed in different regions, since the variability of one wing does not always correlate with the variability of the other. This is confirmed by the lack of correlation of the blue with the red wings, seen in $\sim 34 \%$ of the CTTSs. Each wing tends to be well correlated with itself, indicating that each wing is dominated by a single physical phenomenon.

Acknowledgements. A.P.S. and S.H.P.A. acknowledge support from CNPq, CAPES and Fapemig. J.F.G. aknowledges support from FCT ref project UID/ FIS/04434/2013.

\section{References}

Affer, L., Micela, G., Favata, F., Flaccomio, E., \& Bouvier, J. 2013, MNRAS, 430, 1433

Alencar, S. H. P., \& Batalha, C. 2002, ApJ, 571, 378

Alencar, S. H. P., Teixeira, P. S., Guimarães, M. M., et al. 2010, A\&A, 519, A88

Alencar, S. H. P., Bouvier, J., Walter, F. M., et al. 2012, A\&A, 541, A116

Appenzeller, I., \& Mundt, R. 1989, A\&ARv, 1, 291

Beristain, G., Edwards, S., \& Kwan, J. 2001, ApJ, 551, 1037

Bessolaz, N., Zanni, C., Ferreira, J., Keppens, R., \& Bouvier, J. 2008, A\&A, 478,155

Blinova, A. A., Romanova, M. M., \& Lovelace, R. V. E. 2015, MNRAS, submitted [arXiv: 1501.01948]

Bouvier, J., Covino, E., Kovo, O., et al. 1995, A\&A, 299, 89

Bouvier, J., Alencar, S. H. P., Boutelier, T., et al. 2007a, A\&A, 463, 1017

Bouvier, J., Alencar, S. H. P., Harries, T. J., Johns-Krull, C. M., \& Romanova, M. M. 2007b, Protostars and Planets V, 479

Cauley, P. W., \& Johns-Krull, C. M. 2014, ApJ, 797, 112

Cody, A. M., Stauffer, J. R., Micela, G., Baglin, A., \& CSI 2264 Team 2013, Astron. Nachr., 334, 63

Cody, A. M., Stauffer, J., Baglin, A., et al. 2014, AJ, 147, 82

Costigan, G., Vink, J. S., Scholz, A., Ray, T., \& Testi, L. 2014, MNRAS, 440, 3444

Dahm, S. E. 2008, The Young Cluster and Star Forming Region NGC 2264, ed. B. Reipurth, 966

Dahm, S. E., \& Simon, T. 2005, AJ, 129, 829

Donati, J.-F., Skelly, M. B., Bouvier, J., et al. 2010, MNRAS, 409, 1347

Donati, J.-F., Bouvier, J., Walter, F. M., et al. 2011a, MNRAS, 412, 2454

Donati, J.-F., Gregory, S. G., Alencar, S. H. P., et al. 2011b, MNRAS, 417, 472

Edwards, S., Fischer, W., Kwan, J., Hillenbrand, L., \& Dupree, A. K. 2003, ApJ, 599, L41

Edwards, S., Fischer, W., Hillenbrand, L., \& Kwan, J. 2006, ApJ, 646, 319

Fang, M., van Boekel, R., Wang, W., et al. 2009, A\&A, 504, 461

Ferreira, J., Dougados, C., \& Cabrit, S. 2006, A\&A, 453, 785 
Fúrész, G., Hartmann, L. W., Szentgyorgyi, A. H., et al. 2006, ApJ, 648, 1090 Flaccomio, E., Micela, G., \& Sciortino, S. 2006, A\&A, 455, 903

Giampapa, M. S., Basri, G. S., Johns, C. M., \& Imhoff, C. 1993, ApJS, 89, 321

Gillen, E., Aigrain, S., McQuillan, A., et al. 2014, A\&A, 562, A50

Gilmore, G., Randich, S., Asplund, M., et al. 2012, The Messenger, 147, 25

Gregory, S. G., Donati, J.-F., Morin, J., et al. 2012, ApJ, 755, 97

Gullbring, E., Hartmann, L., Briceño, C., \& Calvet, N. 1998, ApJ, 492, 323

Hartmann, L., Hewett, R., \& Calvet, N. 1994, ApJ, 426, 669

Herbig, G. H. 1954, ApJ, 119, 483

Herbst, W. 1989, AJ, 98, 2268

Herbst, W., Rhode, K. L., Hillenbrand, L. A., \& Curran, G. 2000, AJ, 119, 261

Horne, J. H., \& Baliunas, S. L. 1986, ApJ, 302, 757

Ingleby, L., Calvet, N., Bergin, E., et al. 2011, ApJ, 743, 105

Johns, C. M., \& Basri, G. 1994, in Cool Stars, Stellar Systems, and the Sun, ed. J.-P. Caillault, ASP Conf. Ser., 64, 190

Johns, C. M., \& Basri, G. 1995a, AJ, 109, 2800

Johns, C. M., \& Basri, G. 1995b, ApJ, 449, 341

Johns-Krull, C. M., Valenti, J. A., \& Koresko, C. 1999, ApJ, 516, 900

Johnstone, C. P., Jardine, M., Gregory, S. G., Donati, J.-F., \& Hussain, G. 2014, MNRAS, 437, 3202

Kulkarni, A. K., \& Romanova, M. M. 2008, MNRAS, 386, 673

Kulkarni, A. K., \& Romanova, M. M. 2009, MNRAS, 398, 701

Kurosawa, R., \& Romanova, M. M. 2013, MNRAS, 431, 2673

Kurosawa, R., Harries, T. J., \& Symington, N. H. 2005, MNRAS, 358, 671

Kurosawa, R., Harries, T. J., \& Symington, N. H. 2006, MNARS, 370, 580

Kurosawa, R., Romanova, M. M., \& Harries, T. J. 2008, MNRAS, 385, 1931

Kurosawa, R., Romanova, M. M., \& Harries, T. J. 2011, MNRAS, 416, 2623

Lada, C. J., Muench, A. A., Luhman, K. L., et al. 2006, ApJ, 131, 1574

Lamm, M. H., Bailer-Jones, C. A. L., Mundt, R., Herbst, W., \& Scholz, A. 2004, A\&A, 417, 557
Lima, G. H. R. A., Alencar, S. H. P., Calvet, N., Hartmann, L., \& Muzerolle, J. 2010, A\&A, 522, A104

Luhman, K. L., Briceño, C., Stauffer, J. R., et al. 2003, ApJ, 590, 348

Manara, C. F., Testi, L., Rigliaco, E., et al. 2013, A\&A, 551, A107

McGinnis, P. T., Alencar, S. H. P., Guimarães, M. M., et al. 2015, A\&A, 577, A11

McQuillan, A., Aigrain, S., \& Mazeh, T. 2013, MNRAS, 432, 1203

Meyer, M. R. 2009, in IAU Symp. 258, eds. E. E. Mamajek, D. R. Soderblom, \& R. F. G. Wyse, 111

Muzerolle, J., Hartmann, L., \& Calvet, N. 1998, AJ, 116, 455

Oliveira, J. M., Foing, B. H., van Loon, J. T., \& Unruh, Y. C. 2000, A\&A, 362, 615

Randich, S., Gilmore, G., \& Gaia-ESO Consortium. 2013, The Messenger, 154, 47

Rebull, L. M. 2001, AJ, 121, 1676

Rebull, L. M., Makidon, R. B., Strom, S. E., et al. 2002, AJ, 123, 1528

Reipurth, B., Pedrosa, A., \& Lago, M. T. V. T. 1996, A\&AS, 120, 229

Rucinski, S. M., Matthews, J. M., Kuschnig, R., et al. 2008, MNRAS, 391, 1913 Shu, F., Najita, J., Ostriker, E., et al. 1994, ApJ, 429, 781

Stassun, K. G., Mathieu, R. D., Mazeh, T., \& Vrba, F. J. 1999, AJ, 117, 2941

Stauffer, J., Cody, A. M., Baglin, A., et al. 2014, AJ, 147, 83

Stauffer, J., Cody, A. M., McGinnis, P., et al. 2015, AJ, 149, 130

Sung, H., Bessell, M. S., \& Lee, S.-W. 1997, AJ, 114, 2644

Sung, H., Stauffer, J. R., \& Bessell, M. S. 2009, AJ, 138, 1116

Teixeira, P. S., Lada, C. J., Marengo, M., \& Lada, E. A. 2012, A\&A, 540, A83

Venuti, L., Bouvier, J., Flaccomio, E., et al. 2014, A\&A, 570, A82

Vogt, S. S., Hatzes, A. P., Misch, A. A., \& Kürster, M. 1999, ApJS, 121, 547

Walker, M. F. 1956, ApJS, 2, 365

White, R. J., \& Basri, G. 2003, ApJ, 582, 1109

Zwintz, K., Fossati, L., Ryabchikova, T., et al. 2013, A\&A, 550, A121 


\section{Appendix A: Correlation matrices and $\mathrm{H} \alpha$ line profiles}

We present in this appendix the correlation matrices and $\mathrm{H} \alpha$ line profiles of all the CTTSs we observed with the FLAMES spectrograph during the CSI 2264 campaign. We organized the correlation matrices according to the three main morphologies discussed in Sect. 11. Some correlations matrices are difficult to fit in a single morphological class, and in these cases, we classified the star according to the most representative feature present in the correlation matrix. In Fig. A.1, we grouped all the CTTSs that show anticorrelation in some part of the $\mathrm{H} \alpha$ line profile, like the stars in Fig. 21. A few stars that were not included in this first group present correlation matrices with anticorrelation only in the narrow nebular emission region, near zero velocity. This is due to difficulties in removing the nebular contribution, and the anticorrelation is not related to an emission or absorption region of the star-disk system. In Figs. A.2, we organized the stars that show correlation in almost all of the profile, like the matrix in Fig. 22c. Finally, in Figs. A.3-A.5, we present the stars that show no sign of correlation between the red and the blue wings in most of the profile, like the matrix in Fig. 22a.

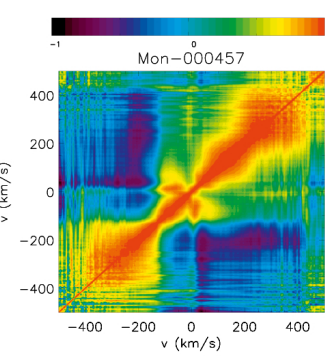

(a)

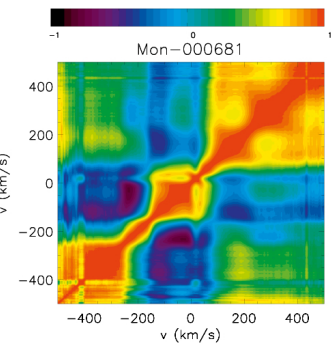

(e)

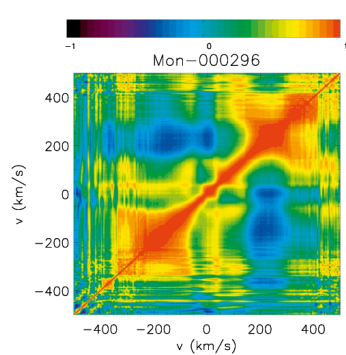

(i)

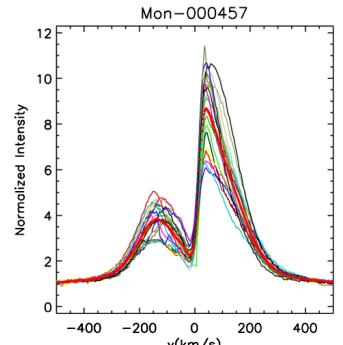

(b)

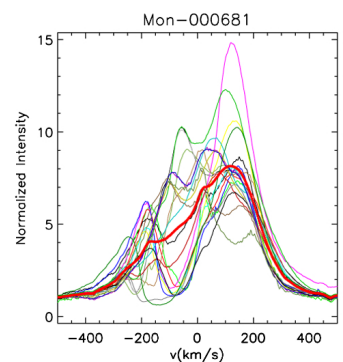

(f)

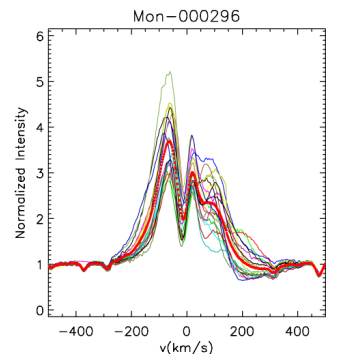

(j)

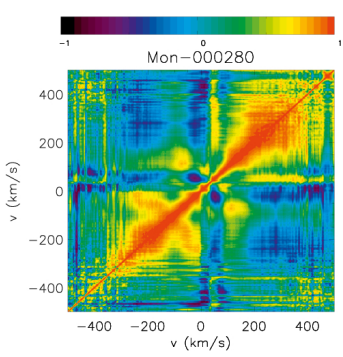

(m)

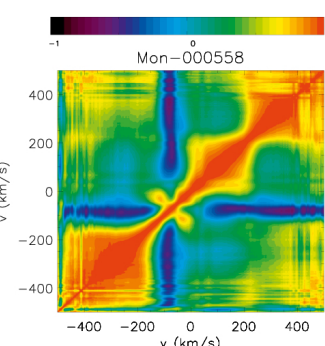

(c)

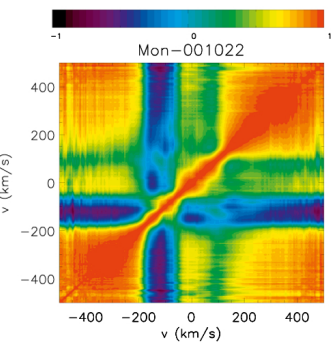

(g)

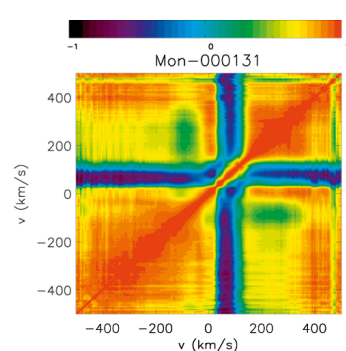

(k)

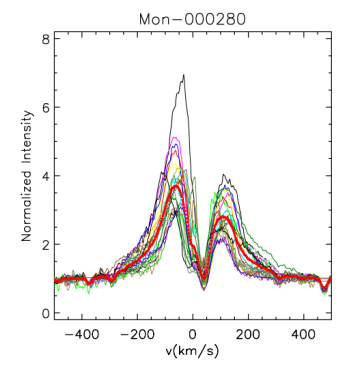

(n)

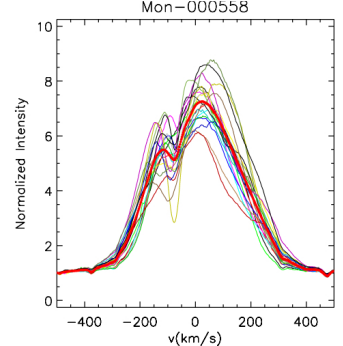

(d)

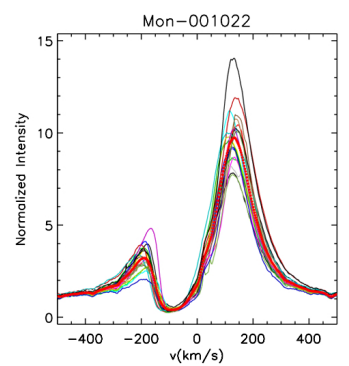

(h)

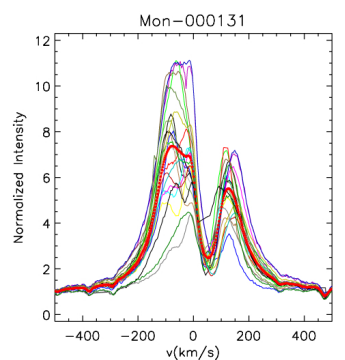

(l)

Fig. A.1. Correlation matrices (left) of the $\mathrm{H} \alpha$ line of selected CTTSs and the corresponding line profiles (right). In the left panels, the color range corresponds to the value of the linear correlation coefficient, $r(i, j)$, between different velocity bins $(i$ and $j)$, of the H $\alpha$ line profiles, as described in Sect. 11. Perfect anticorrelation corresponds to -1 (black), no correlation to 0 (light blue), and a perfect correlation to 1 (orange). When $i=j$, $r(i, j)=1$, and for all values of $i$ and $j, r(i, j)=r(j, i)$, implying that the matrix is symmetrical relative to the diagonal. In the right panels, different colors correspond to spectra observed in different nights, and the thick red line is the average line profile. The stars in this group present anticorrelation of some part of the line profile. The anticorrelation seen in these stars is associated with blue- or redshifted absorption or emission that varies in antiphase with the rest of the profile. For example, when in panel a (Mon-000457) the red wing emission increases in intensity, the blue wing emission decreases. 


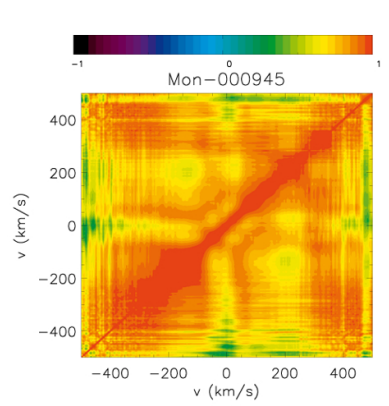

(a)

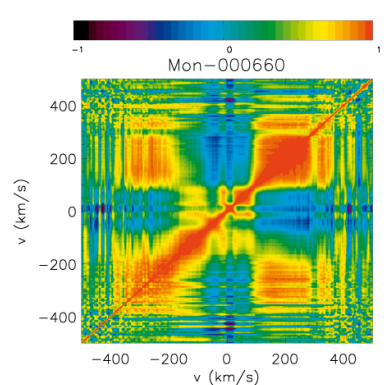

(e)

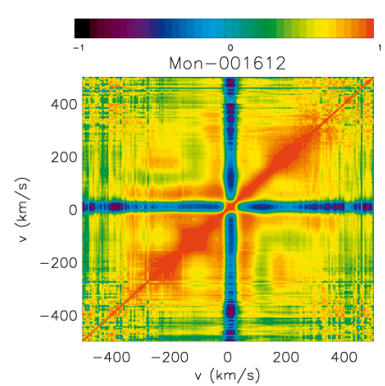

(i)

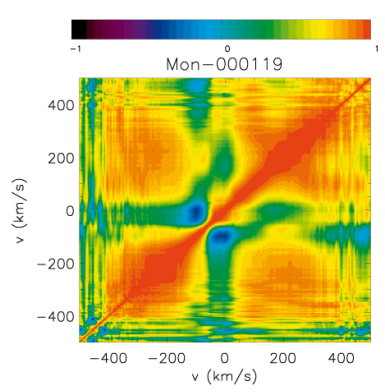

(m)

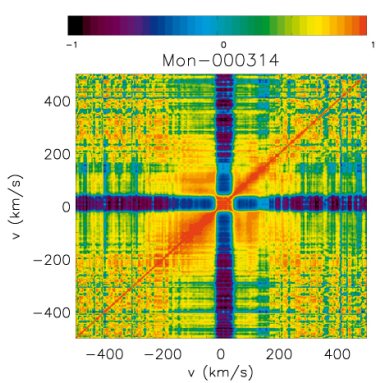

(q)

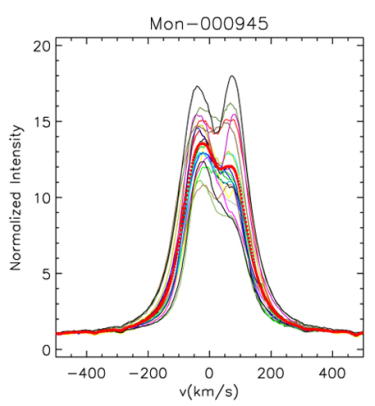

(b)

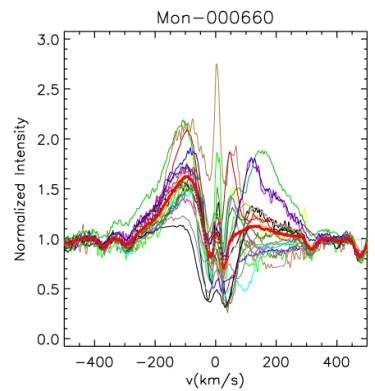

(f)

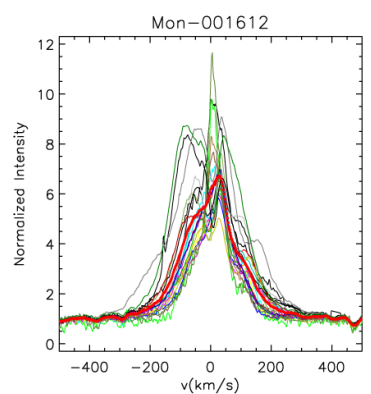

(j)

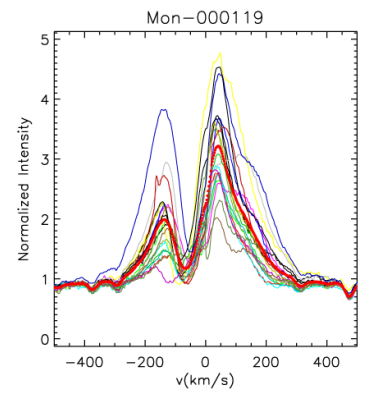

(n)

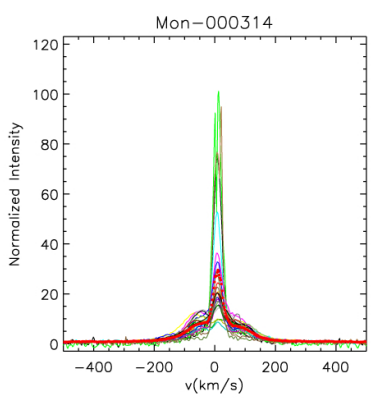

(r)

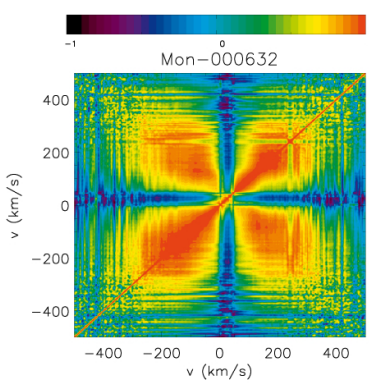

(c)

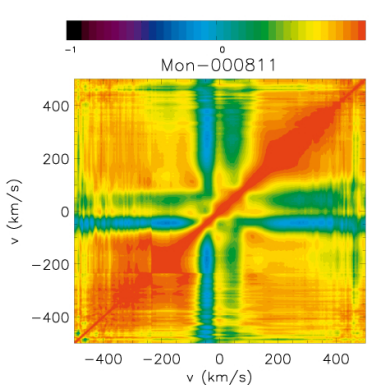

(g)

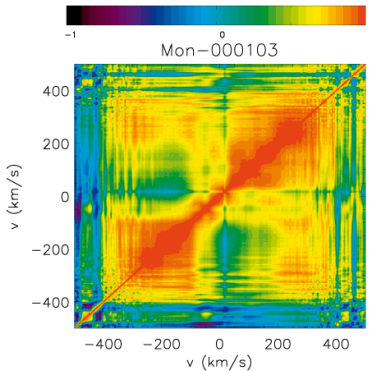

(k)

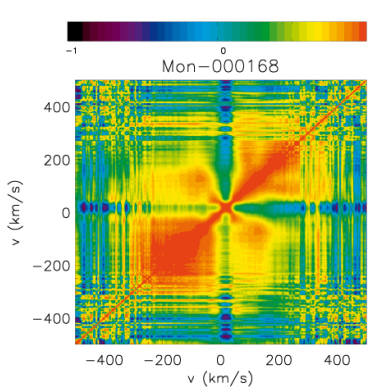

(o)

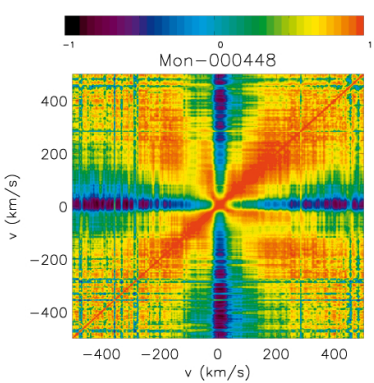

$(\mathrm{s})$

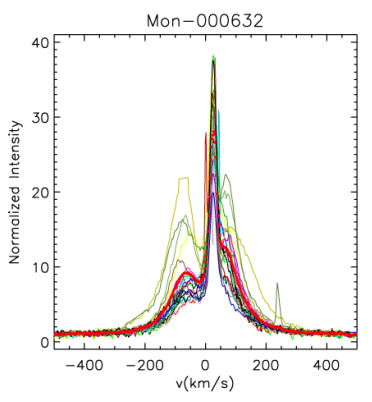

(d)

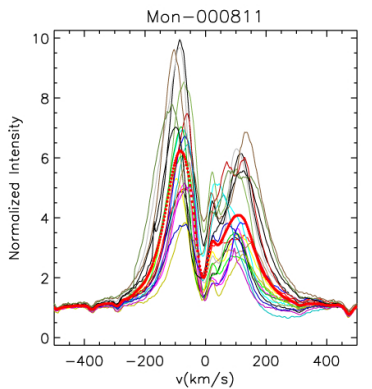

(h)

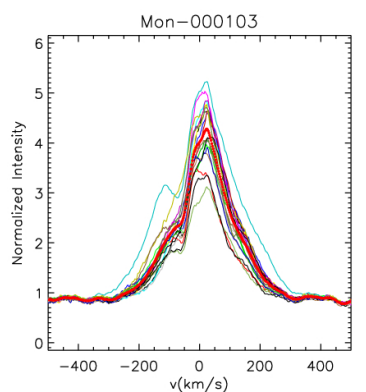

(1)

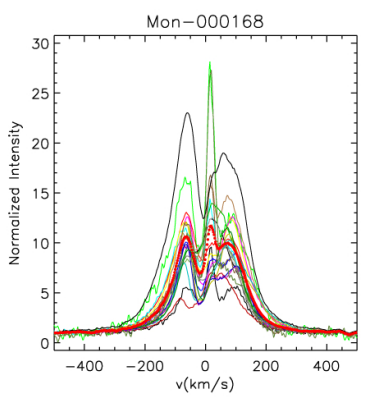

(p)

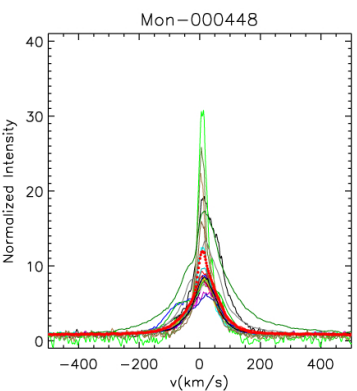

(t)

Fig. A.2. Same as Fig. A.1, but for stars that present strongly correlated line profiles, as indicated by the positive correlation in almost the entire line profile. The narrow anticorrelation region in the matrices of Mon-000632, Mon-001612, Mon-000119, Mon-000314, and Mon-000448 is due to the nebular contribution that was not entirely removed. 
A. P. Sousa et al.: CSI 2264: Accretion process in classical T Tauri stars in the young cluster NGC 2264

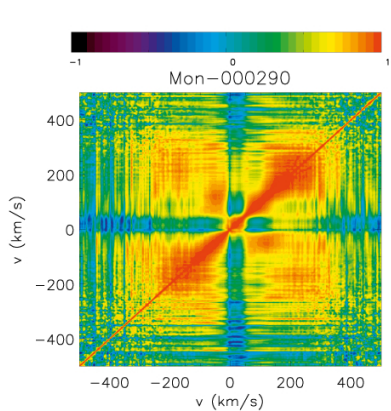

(a)

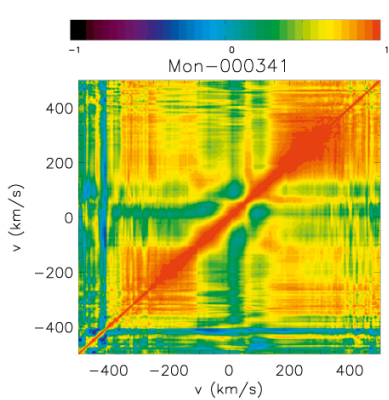

(e)

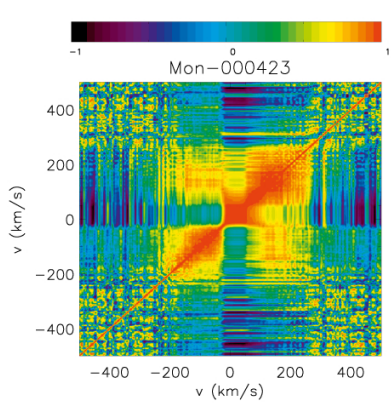

(i)

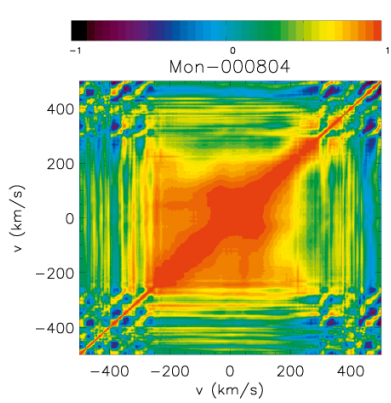

(m)

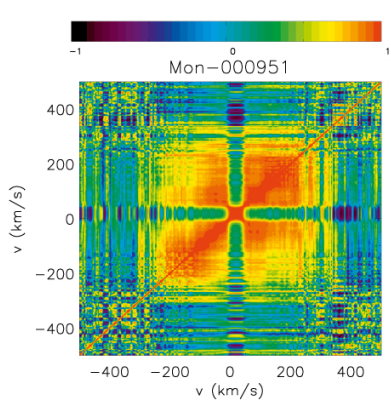

(q)

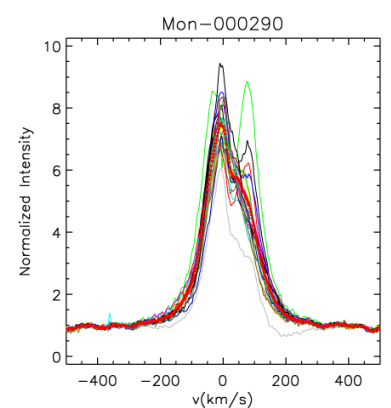

(b)

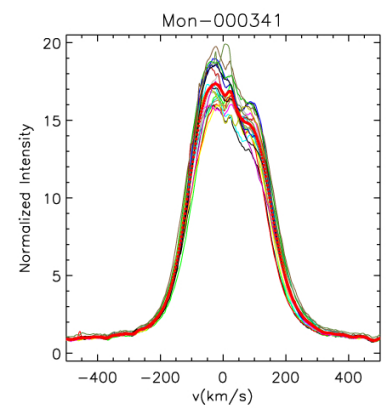

(f)

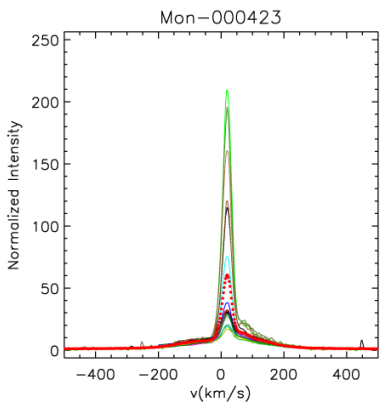

(j)

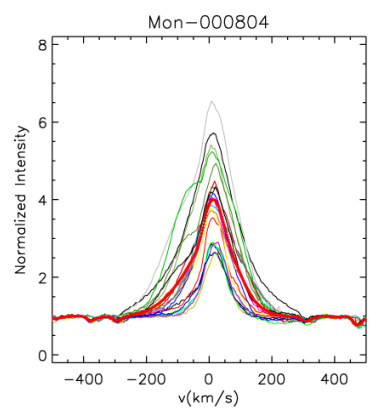

(n)

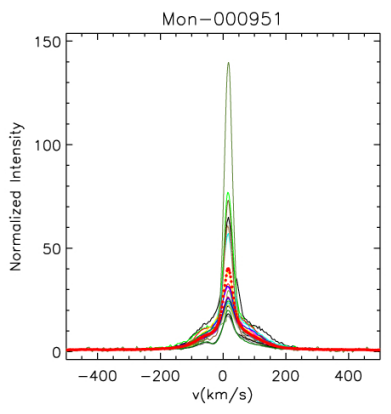

(r)

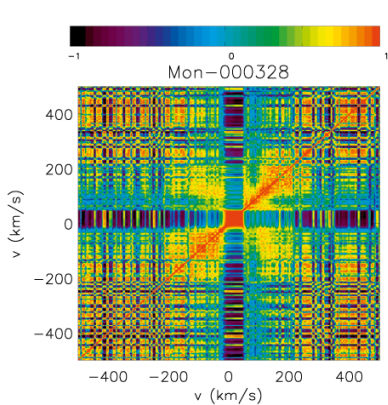

(c)

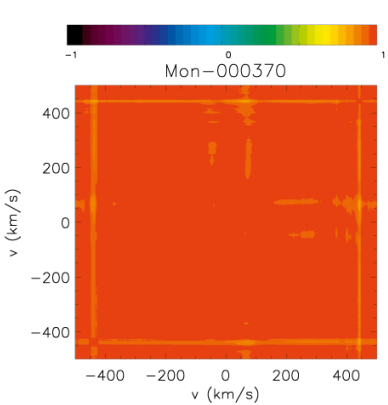

(g)

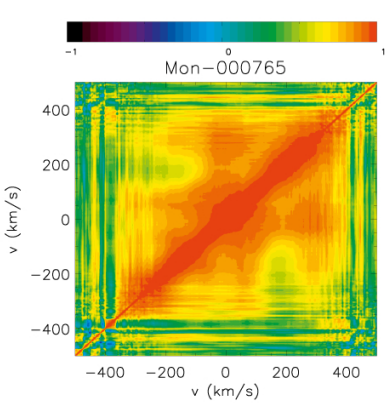

(k)

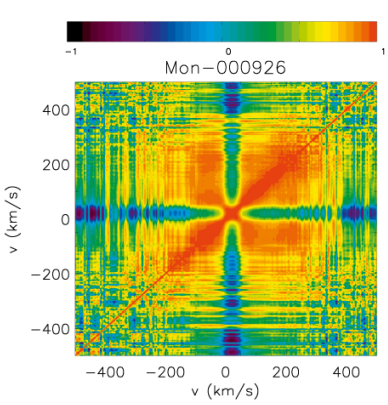

(o)

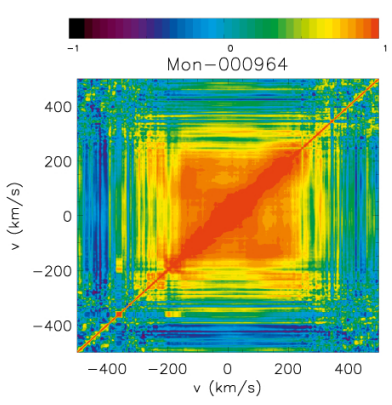

(s)

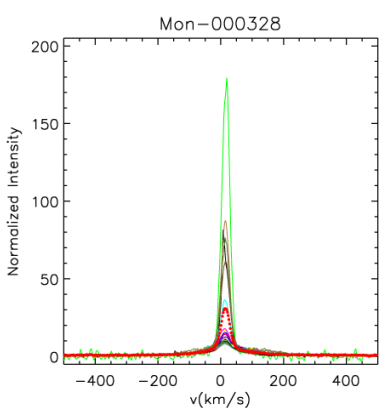

(d)

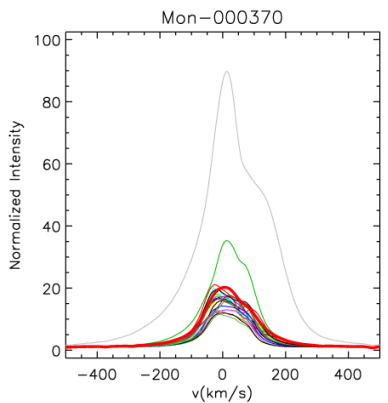

(h)

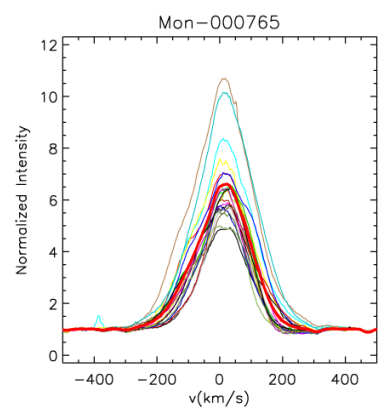

(1)

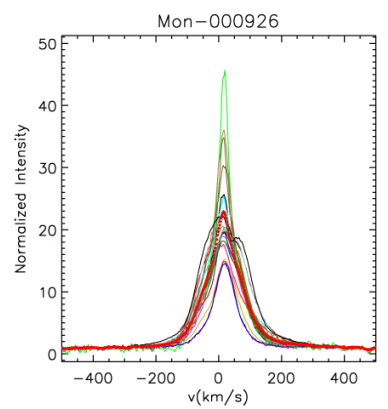

(p)

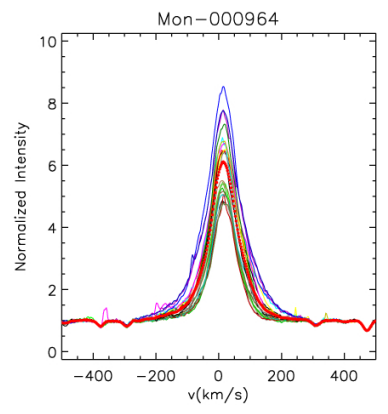

$(\mathrm{t})$

Fig. A.2. continued. 
A\&A 586, A47 (2016)

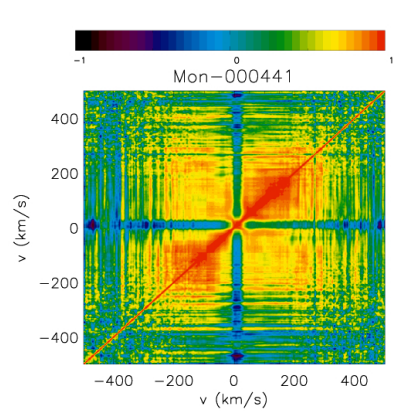

(a)

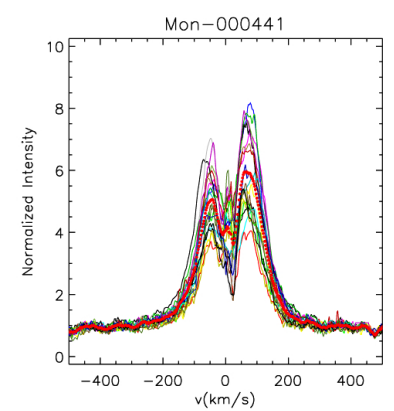

(b)

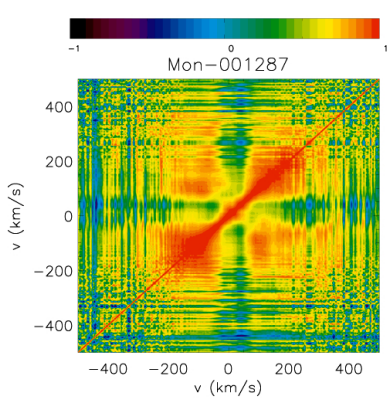

(e)

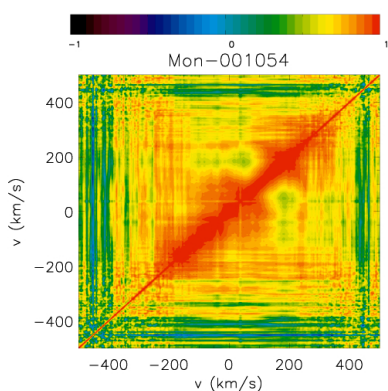

(c)

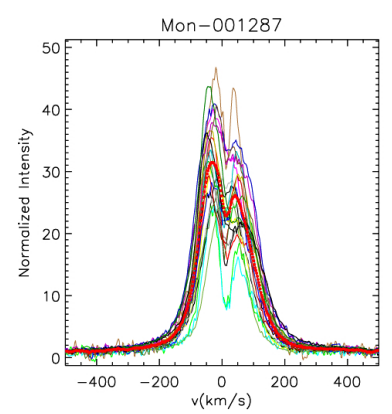

(f)

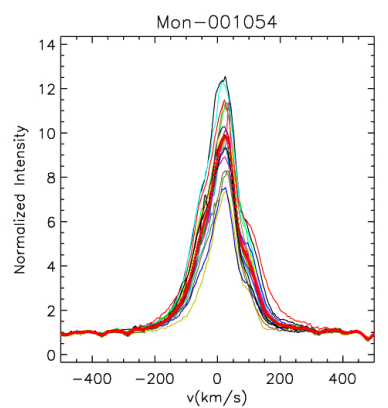

(d)

Fig. A.2. continued. 
A. P. Sousa et al.: CSI 2264: Accretion process in classical T Tauri stars in the young cluster NGC 2264

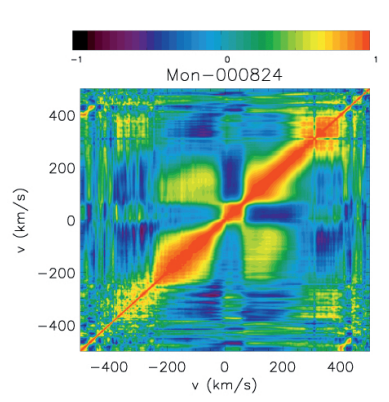

(a)

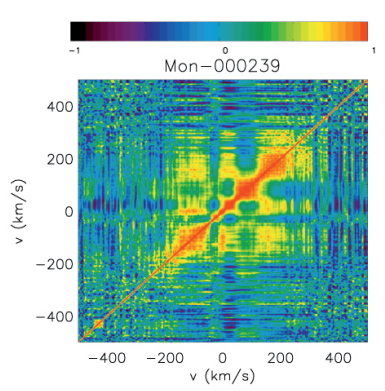

(e)

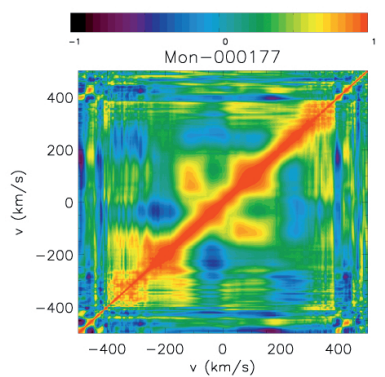

(i)

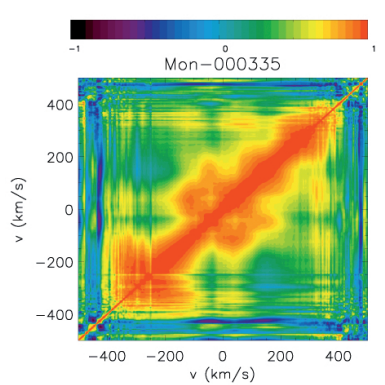

(m)

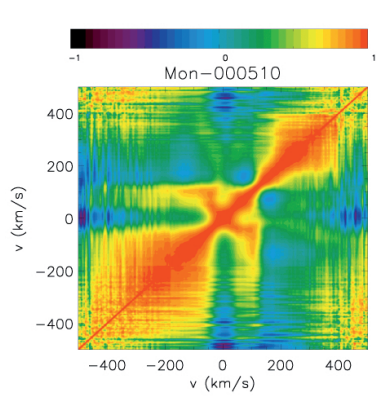

(q)

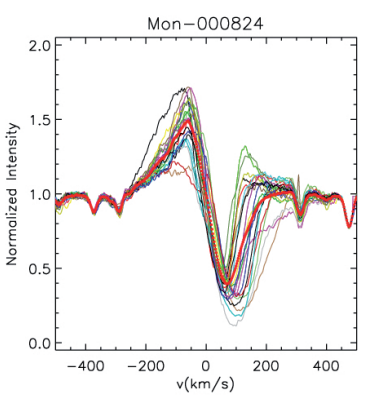

(b)

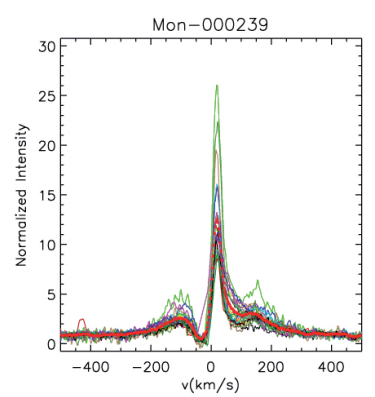

(f)

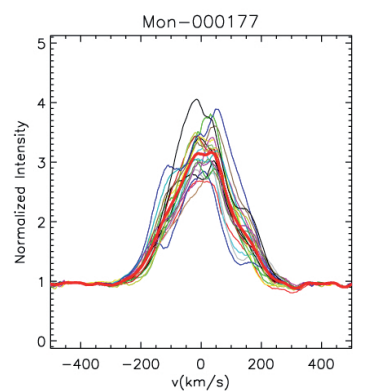

(j)

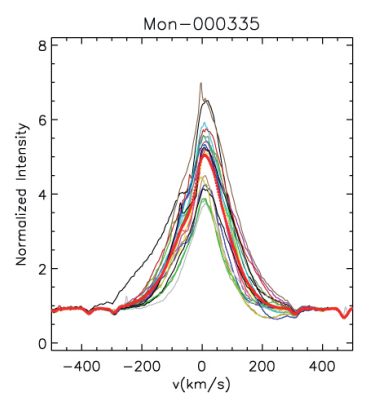

(n)

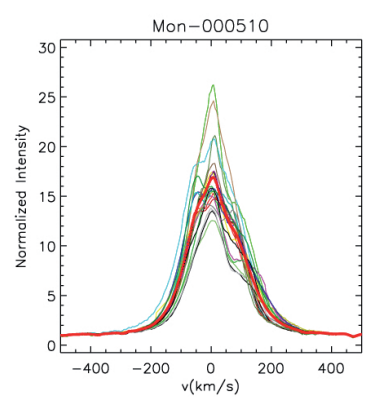

(r)

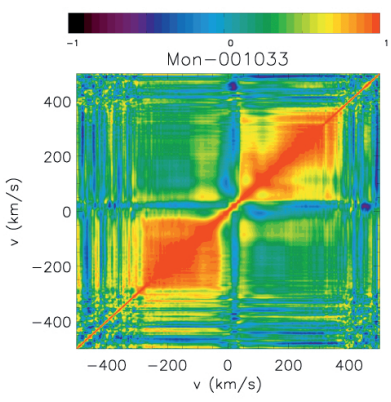

(c)

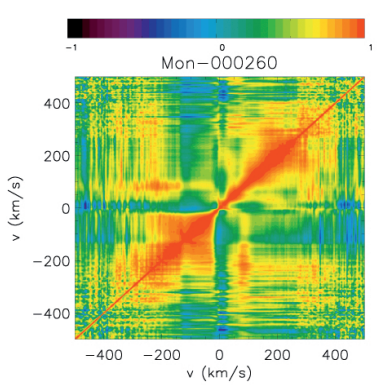

(g)

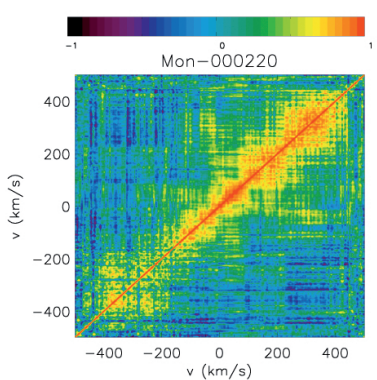

(k)

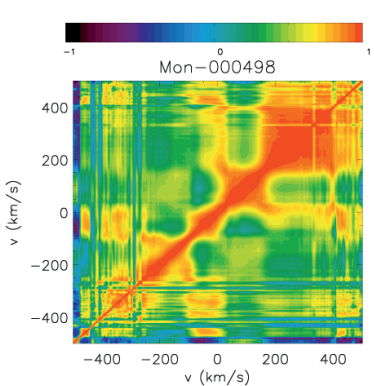

(o)

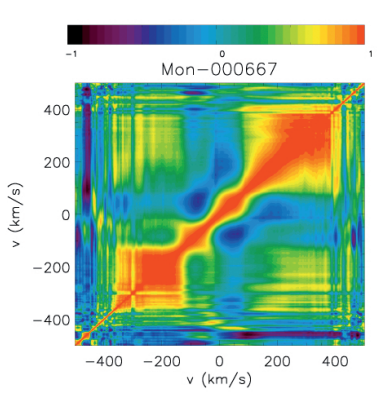

(s)

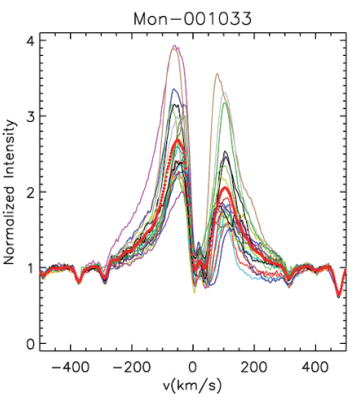

(d)

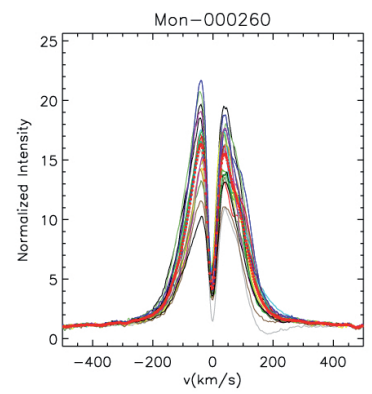

(h)

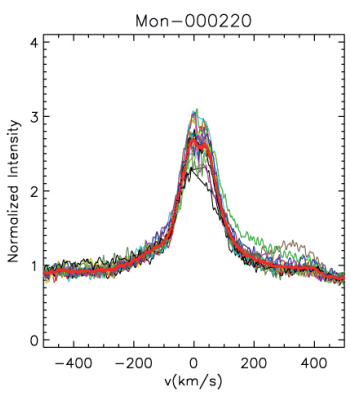

(1)

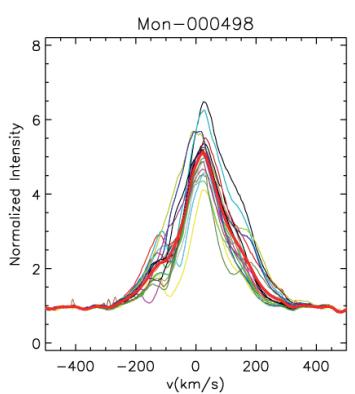

(p)

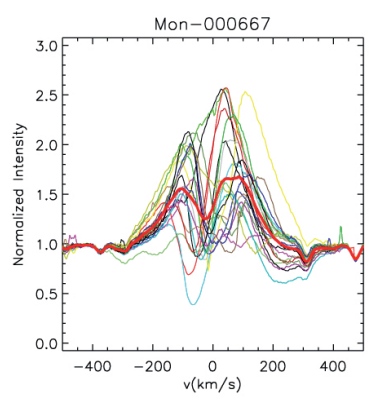

(t)

Fig. A.3. Same as Fig. A.1, but for stars that show no sign of correlation between the red and blue wings of the H $\alpha$ line profile. The narrow anticorrelation in the matrices of Mon-000824, Mon-000177, and Mon-000667 is due to the nebular contribution that was not entirely removed. 


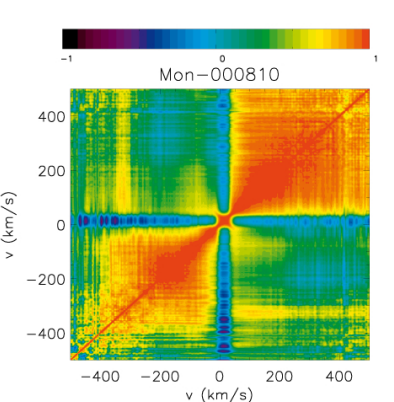

(a)

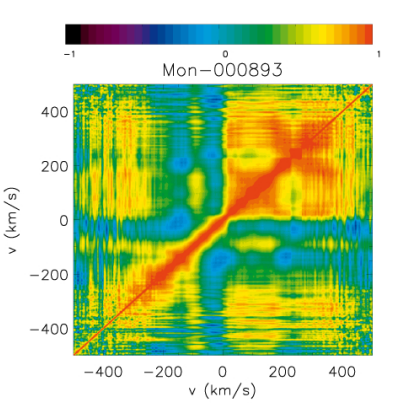

(e)

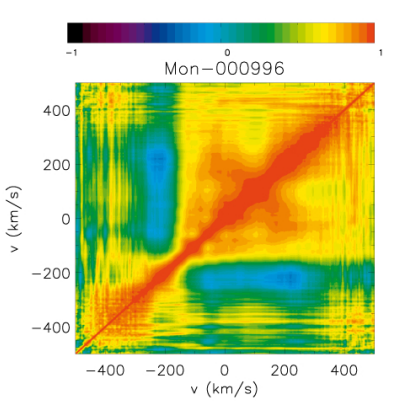

(i)

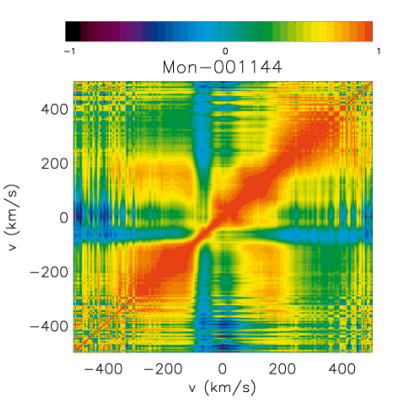

(m)

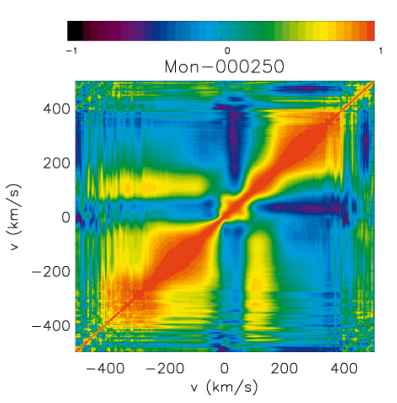

(q)

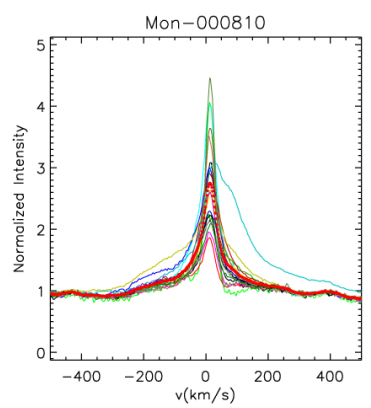

(b)

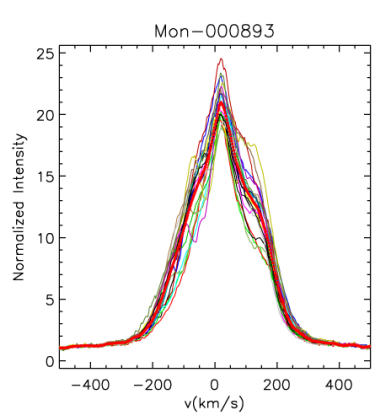

(f)

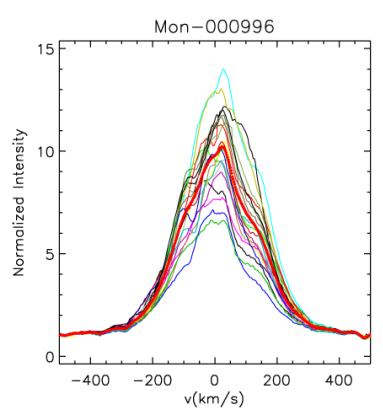

(j)

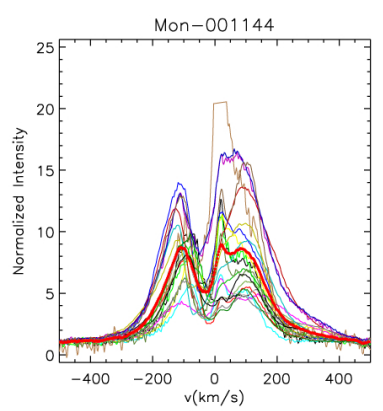

(n)

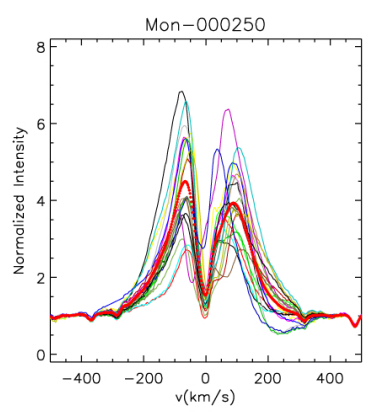

(r)

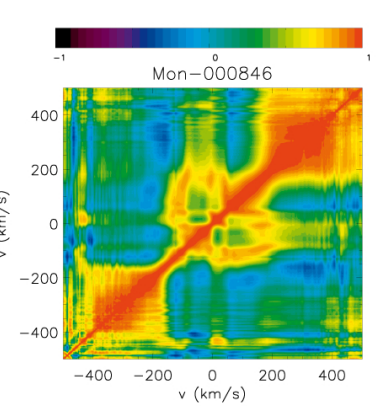

(c)

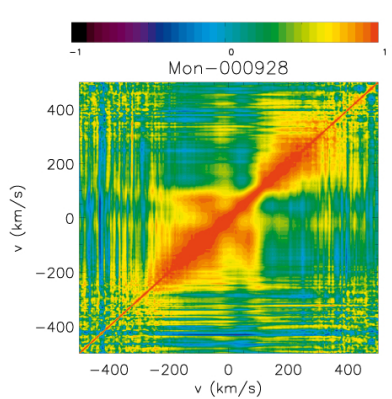

(g)

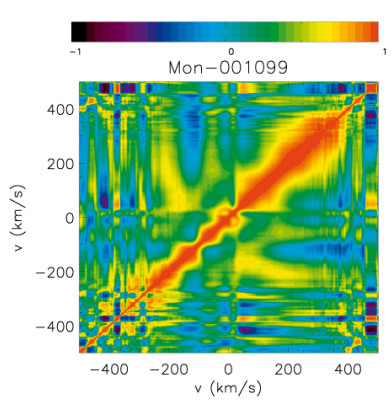

(k)

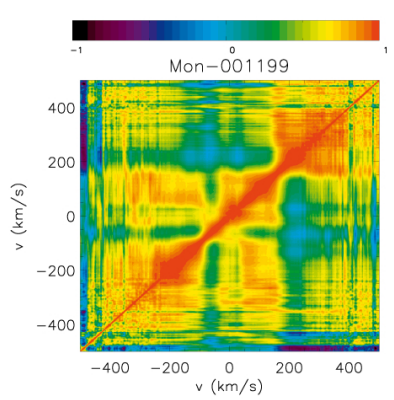

(o)

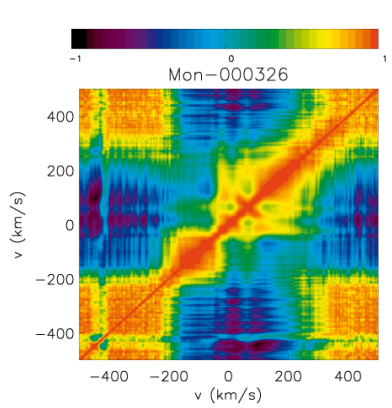

(s)

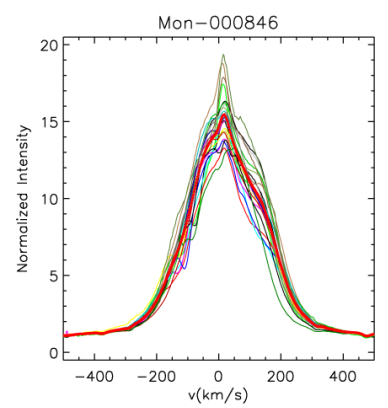

(d)

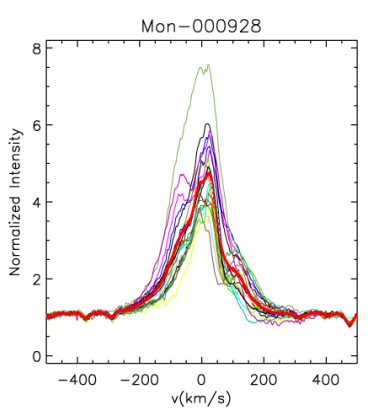

(h)

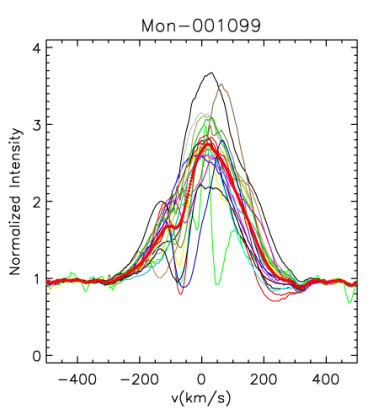

(1)

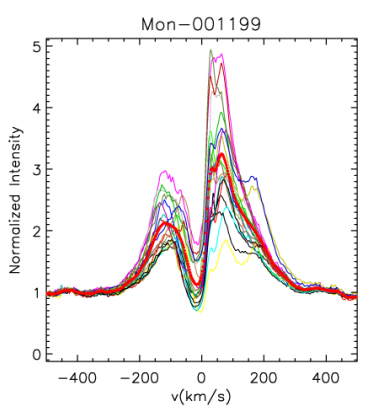

(p)

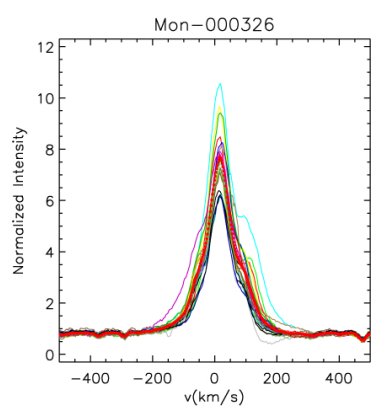

(t)

Fig. A.4. Same as Fig. A.3. The anticorrelation in the matrix of Mon-000250 is due to the nebular contribution that was not entirely removed. 
A. P. Sousa et al.: CSI 2264: Accretion process in classical T Tauri stars in the young cluster NGC 2264

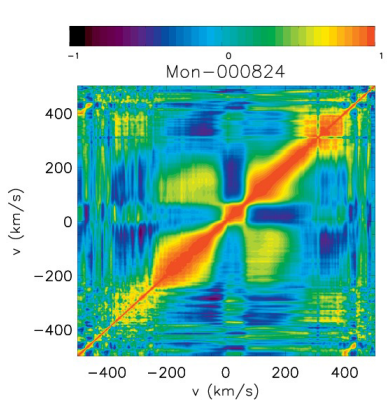

(a)

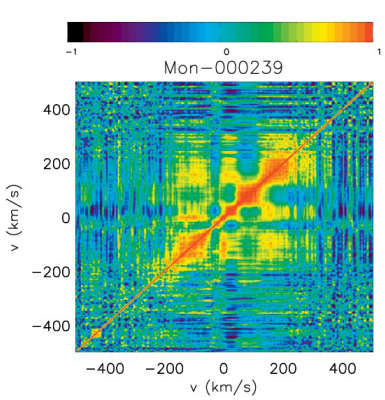

(e)

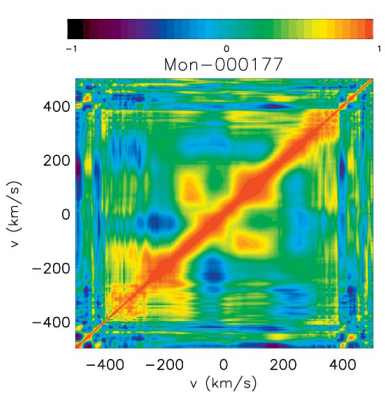

(i)

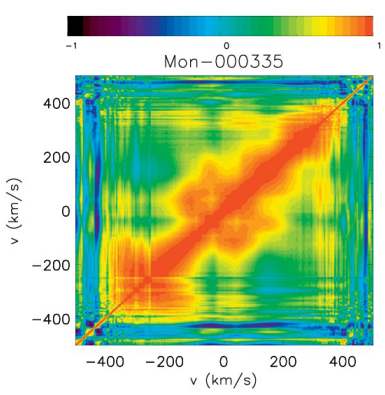

(m)

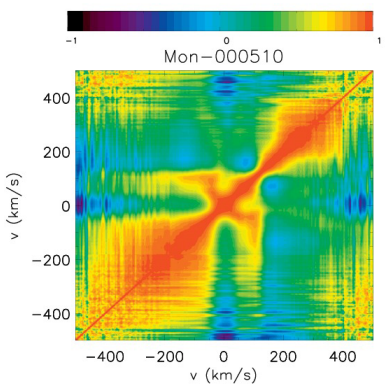

(q)

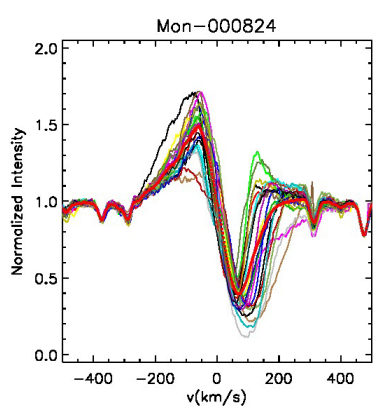

(b)

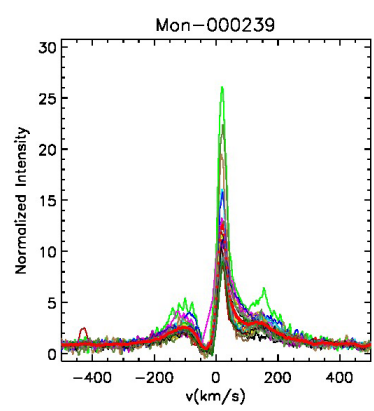

(f)

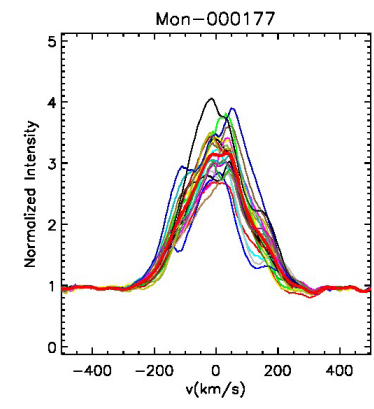

(j)

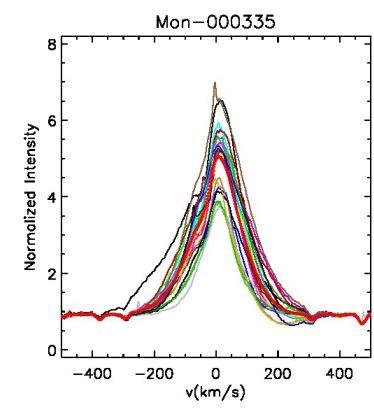

(n)

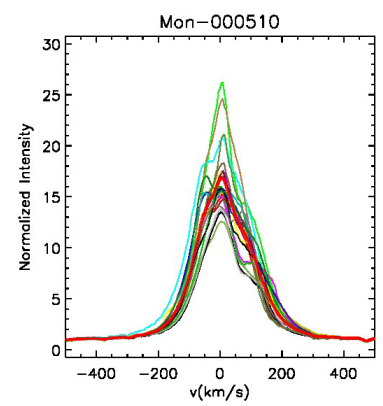

$(\mathrm{r})$

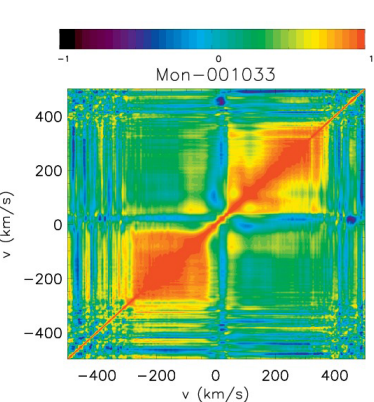

(c)

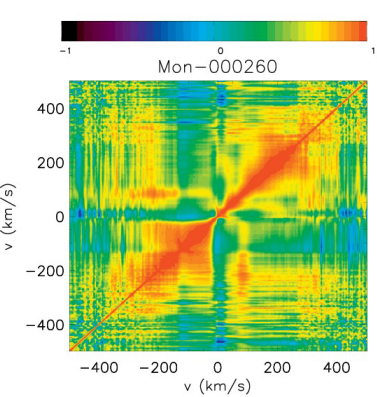

(g)

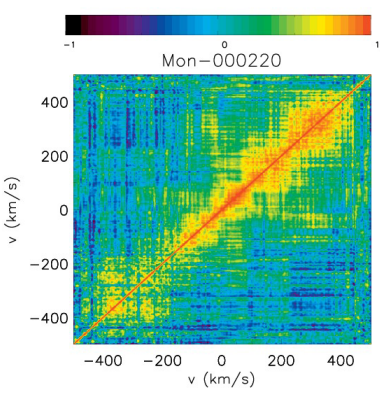

(k)

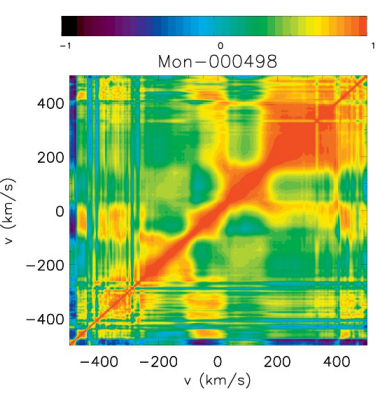

(o)

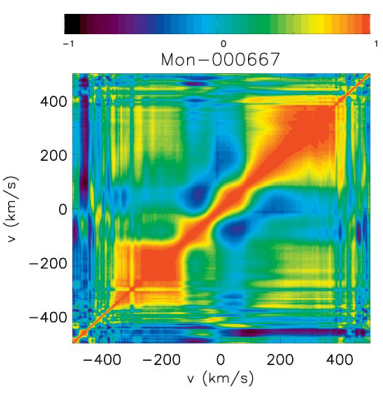

(s)

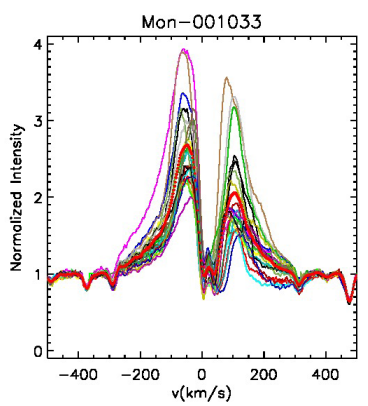

(d)

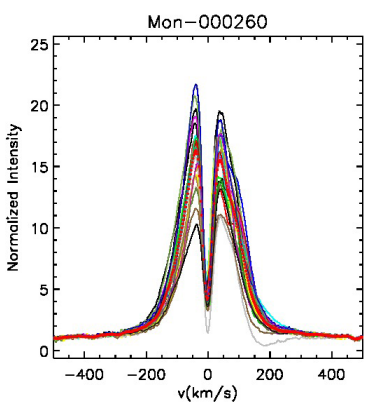

(h)

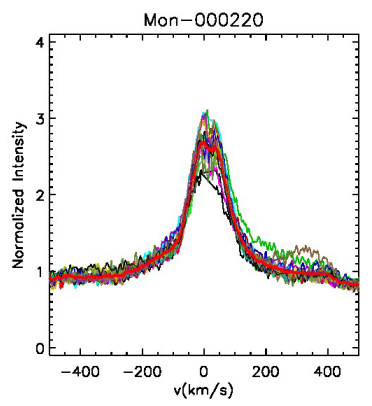

(1)

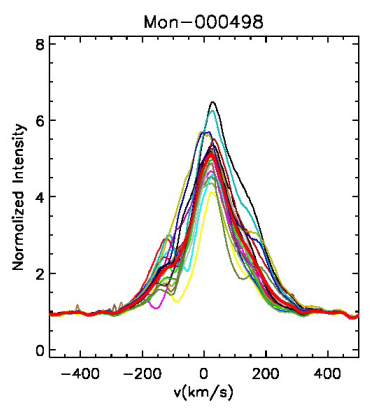

(p)

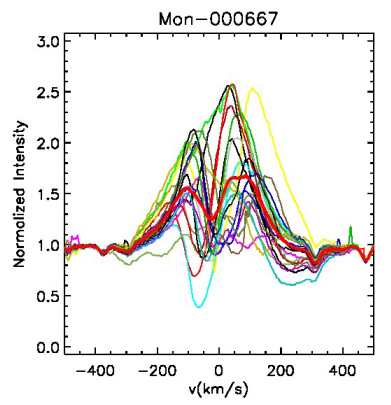

(t)

Fig. A.5. Same as Fig. A.3. The anticorrelation in the matrix of Mon-000795 is due to the nebular contribution that was not entirely removed. 